\title{
Quantum field kinetics of QCD: Quark-gluon transport theory for light-cone-dominated processes
}

\author{
Klaus Geiger* \\ CERN TH-Division, CH-1211 Geneva 23, Switzerland
}

(Received 20 July 1995)

\begin{abstract}
A quantum-kinetic formalism is developed to study the dynamical interplay of quantum and statisticalkinetic properties of nonequilibrium multiparton systems produced in high-energy QCD processes. The approach provides the means to follow the quantum dynamics in both space-time and energy-momentum, starting from an arbitrary initial configuration of high-momentum quarks and gluons. Using a generalized functional integral representation and adopting the "closed-time-path" Green function techniques, a self-consistent set of equations of motions is obtained: a Ginzburg-Landau equation for a possible color background field, and Dyson-Schwinger equations for the two-point functions of the gluon and quark fields. By exploiting the "two-scale nature" of light-cone-dominated QCD processes, i.e., the separation between the quantum scale that specifies the range of short-distance quantum fluctuations, and the kinetic scale that characterizes the range of statistical binary interactions, the quantum field equations of motion are converted into a corresponding set of "renormalization equations" and "transport equations." The former describe renormalization and dissipation effects through the evolution of the spectral density of individual, dressed partons, whereas the latter determine the statistical occurrence of scattering processes among these dressed partons. The renormalization equations and the transport equations are coupled, and, hence, must be solved self-consistently. This amounts to evolving the multiparton system, from a specified initial configuration, in time and full seven-dimensional phase space, constrained by the Heisenberg uncertainty principle. This quantum-kinetic description provides a probabilistic interpretation and is, therefore, of important practical value for the solution of the dynamical equations of motion, suggesting, for instance, the possibility of simulating the multiparticle dynamics with Monte Carlo methods. [S0556-2821(96)01113-7]
\end{abstract}

PACS number(s): 12.38.Aw, 12.38.Cy, 13.38.Mh

\section{INTRODUCTION}

In this paper I attempt to formulate an approach towards a fundamental and consistent description of the statistical properties of nonequilibrium quantum systems produced in high-energy QCD processes, which allows us to follow the quantum dynamics in time and complete phase space starting from an initial configuration. It provides a flexible framework for a systematic analysis of typical problems associated with the quantum dynamics of such systems, including, e.g., multiparticle transport phenomena of gluons, quarks, and hadrons, or critical dynamics of phase-transition phenomena and spontaneous symmetry breaking, or quantum dissipation, entropy generation, and multiparticle production.

More specifically, the intentions are aimed towards a practically applicable description of the space-time evolution of a general initial system of gluons and quarks, characterized by some large energy or momentum scale, that expands, diffuses, and dissipates according to the self- and mutual interactions, and eventually converts dynamically into excited hadronic matter and a final state hadron system by a "phase transition." This scenario frames a wide class of QCD processes of both fundamental and phenomenological interest. For instance, the evolution of parton showers in the mechanism of parton-hadron conversion in elementary highenergy processes $\left(e^{+} e^{-}\right.$annihilation into hadrons, deep in-

*Electronic address: klaus@surya11.cern.ch elastic lepton-nucleon scattering, or nondiffractive hadronic collisions), or the description of formation, evolution, and freeze-out of a quark-gluon plasma in ultrarelativistic heavyion collisions, or the study of the dynamics of the QCD phase transition from the deconfined, high-temperature partonic phase to a low-temperature hadronic phase with the simultaneous breakdown of chiral symmetry and the condensation of gluons and quarks in the vacuum, as it occurred during the early evolution of the Universe.

In the present paper I will confine myself to the first stage, the high-energy quark-gluon phase, and develop a quantumkinetic formalism that allows one to describe both the dissipative and dispersive dynamics of a multiparton system in real time. This description is exclusively based on the fundamental QCD Lagrangian and its firmly established principles. The second stage, the parton-hadron conversion and phase transition, on the other hand, requires supplementary phenomenological input to model the details of the confinement mechanism that are not known at present [1]. Such a phenomenological approach to the real-time dynamics of parton-hadron conversion that models the transition within an effective field theory description has been proposed recently in Ref. [2]. It is preferable, however, to keep the fundamental description of the first stage distinct from the less understood phenomenological aspects of the second stage, and, therefore, I will address the latter in a separate paper.

In general, the study of a high-energy multiparticle system and its quantum dynamics involves three essential aspects: first, the aspect of space-time, geometry, and the structure of the vacuum; second, the quantum field aspect of the 
particle excitations; and third, the statistical aspect of their interactions. These three elements are generally interconnected in a nontrivial way by their overall dynamical dependence. Therefore, in order to formulate a quantum description of the complex nonequilibrium dynamics, one needs to find a quantum-statistical and kinetic formulation of field theory that unifies the three aspects self-consistently. With this paper I take steps towards this goal by combining three corresponding theoretical methods, namely, first, the closedtime-path (CTP) formalism [3-9] (for treating initial value problems of irreversible systems), second, the nonlocal source theory [10-12] (for incorporating quantum fluctuations), and third, transport theory based on Wigner function techniques [13-15] (for a kinetic description of inhomogeneous nonequilibrium systems). In principle, a dynamical theory of nonequilibrium multiparticle systems such as the above mentioned should be described by an exact quantumkinetic theory of QCD. Over the past ten years, elaborate works [16-18] have put great effort into deriving a general QCD transport theory rigorously from first principles. Unfortunately, due to a number of unresolved problems arising from the complexities of the non-Abelian gauge structure of $\mathrm{QCD}$, the derived gauge-covariant formalism remains an academic theory up to date. It is of little practical value, unless it is boiled down to the quasiclassical limit by a series of approximations yielding a mean-field description, which, however, cannot describe the production of physical particles and their spectra.

I am less ambitious here in what concerns the generality, and instead put emphasis on applicability to realistic physical situations, in particular to the type of light-cone-dominated processes that I classified above. This class of high-energy processes allows a clear distinction between a short-distance quantum field theoretical scale and a larger distance statistical-kinetic scale. When described in a reference frame in which the particles move at close to the speed of light, the effects of time dilation and Lorentz contraction separate the intrinsic quantum motion of the individual particles from the statistical correlations among them. On the one hand, the quantum dynamics is determined by the self-interactions of the bare quanta, and by the possible presence of a coherent background field (or mean field in the Hartree-Fock sense), in case one desires to go beyond a description in the pure vacuum. This requires a fully quantum theoretical analysis including renormalization. On the other hand, the kinetic dynamics can be well described statistical-mechanically by the motion of the quasiparticles which arise from the "dressing", of the bare quanta by their self-interactions and by the background field, plus the binary interactions between these quasiparticles. Such a distinct description of quantum and kinetic dynamics is possible, because the quantum fluctuations are highly concentrated around the light cone, occurring at very short distances, and decouple to very good approximation from the kinetic evolution which is dictated by comparably large space-time scales. As mentioned, the natural twoscale separation is just the consequence of time dilation and Lorentz contraction, and is true for any light-cone-dominated process. In fact, at asymptotic energies the quantum fluctuations are exactly localized on the light cone, and so the decoupling becomes perfect. This observation is the key to formulating a quantum-kinetic description in terms of particle phase-space densities, involving a simultaneous specification of momentum space and space-time, because at sufficiently high energy the momentum scale $\Delta p$ of the individual particles' quantum fluctuations and the scale $\Delta r$ of space-time variations of the system of particles satisfy $\Delta p \Delta r \gg 1$, consistent with the uncertainty principle.

With this physical input and utilizing the aforementioned theoretical tools, the analysis proceeds as follows. In the first step, covered in Sec. II, I obtain, starting from the QCD Lagrangian, the CTP generating functional for the gluon and quark Green functions, being defined on a closed-time contour and incorporating initial state correlations. From the associated effective action, one gets the quantum-dynamical equations of motion, which are the CTP version of the Ginzburg-Landau equation and the Dyson-Schwinger equations. In the second step, described in Sec. III, I make the transition from a quantum field description to kinetic theory, by exploiting the two-scale nature of light cone dominance, and, moreover, choosing a ghost-free axial gauge for the gluon fields. As a result one obtains from the DysonSchwinger equations a set of kinetic equations consisting of a renormalization equation that describes the quantum dynamics in terms of short-distance self-interactions of gluons and quarks, plus a transport equation that describes the kinetic dynamics of relaxation and collision processes in terms of the statistical interactions of the renormalized, dynamically dressed partons among one another. The renormalization equation and the transport equations are coupled, and hence must be solved self-consistently. This amounts to evolving the system under consideration from its initial configuration simultaneously in position and momentum space, constrained by the Heisenberg uncertainty principle. Finally, Sec. IV closes with some concluding remarks, and the Appendixes summarize, for each of the above aspects, the technical details which are only indicated in the text.

The main findings can be summarized as follows. The dynamics of high-energy multiparton systems can, under reasonable conditions, be described in a semiclassical manner: the partons can be considered as dressed quanta with a dynamical substructure and a corresponding form factor arising from the self-interactions. The space-time evolution of a system of many such dressed partons is then governed by their propagation along classical trajectories and mutual binary collisions, as determined by their density and cross sections, and by quantum statistics. This emerging picture is of great practical value for formulating a systematic calculation scheme-in a sense the space-time generalization of the "jet calculus" $[19,20]$. In Sec. III F, I outline such a scheme. One of the greatest advantages of this kinetic description is that it provides a probabilistic interpretation of the time evolution in full seven-dimensional phase space, which suggests the opportunity to simulate the multiparticle dynamics as sequential Markov processes with Monte Carlo methods.

Finally let me comment on placing this work in relation to existing literature.

(i) The general ideas and techniques of the CTP functional integral formalism were originally introduced mainly by Schwinger [3], Keldysh [4], Kadanoff and Baym [5], and Mahanthappa [6], more than 30 years ago. The most extensive review that sums up the current state of the art is probably the work of Chou et al., [7] with diverse exemplification 
of the wide class of physics applications. Further pedagogically excellent presentations have been published by Calzetta and $\mathrm{Hu}$ [8], and by Rammer and Smith [9]. In the particular field of relativistic nuclear physics, the concepts have been pragmatically applied, e.g., by Li and McLerran [21], and by Zhang and Wilets [22]. Important contributions of fundamental studies have been made in recent years by Danielewicz [23], and Mrówczyńsky and Heinz [24]. The goal of establishing a quantum kinetic theory for QCD was pioneered by the ambitious efforts of Elze, Gyulassy, Heinz, and Vasak [16-18], which resulted in a rigidly general, gauge-covariant formalism. However, the price to pay is an intractable complexity that, without specific physics input, is essentially of aesthetic value without much practical use. The new achievement of the present work from this perspective may be stated as the adaption of the general CTP formalism, applied to QCD, but with focus on situations where the multiparton dynamics is characterized by a large energy scale and can be described reliably within perturbation theory in a physical gauge.

(ii) The most related recent works from the viewpoint of attempting to tackle evolution of multiparton systems at high energy are probably the innovative works of McLerran and Venugopalan and co-workers [25], and of Makhlin [26], in which the issue of calculating parton distributions in the context of ultrarelativistic nuclear collisions is addressed. The former authors use a classical non-Abelian field description of QCD to compute coherent initial state properties of colliding large nuclei, whereas the latter focuses on a quantum field description of final state correlations of the particles produced. Neither approach, however, attempts to address explicitly the space-time evolution of the multiparton ensemble emerging from the nuclear collision, which is the main goal of the present paper.

(iii) The key elements to address the space-time evolution are provided by the Wigner function techniques, which date back to Wigner's work on transport phenomena [13], and are reviewed in, in e.g., $[14,15]$. Although widely exploited in condensed matter and plasma physics, these tools for quantum kinetics of many-body systems have hardly been applied to describe high-energy nonequilibrium dynamics in QCD. New in the present work is the synthesis of quantum dynamics on the basis of the renormalization group of QCD, and quasiparticle kinetics within relativistic transport theory. The combination of these two aspects forms the foundation of the self-consistent treatment that entails a thorough consideration of the renormalization problem, which is commonly avoided in other applications (see, however, Ref. [8]).

(iv) The machinery of perturbative QCD for light-conedominated high-energy processes is nowadays well founded. Most of the techniques used in the perturbative analysis to describe the parton evolution adopt the tools developed by Dokshitzer et al. [27], Amati et al. [28], Mueller [29], and numerous others (for an overview, see [30,31]). The new component here is the extension to incorporate a space-time description on top of this formalism, which is commonly considered only in momentum space.

It is evident that this paper attempts to join theoretical tools and concepts from rather different fields. Such a synthesis is necessarily a difficult task, and the the present initiative should be viewed as a first step in this direction. How- ever, I believe that it is a promising approach towards a well-founded and consistent description of the statistical properties of nonequilibrium parton systems. From the phenomenological perspective, it is an inevitable necessity to address this problem, since the experiments carried out at the DESY $c p$ collider HERA, BNL Relativistic Heavy Ion Collider (RHIC), and CERN Large Hadron Collider (LHC) will penetrate increasingly the physics of high-density QCD, where quark-gluon transport phenomena are of fundamental importance.

\section{FUNCTIONAL FORMALISM}

The aim is to describe the time evolution of a general nonequilibrium quantum system consisting of an ensemble of quarks and gluons in phase space, starting from some given initial state at time $t_{0}$. Since I am interested in the state of the system at finite times $t>t_{0}$, without $a$ priori knowledge of the asymptotic final state at $t=\infty$, the usual $S$-matrix formalism of quantum field theory, based on in-out matrix elements, cannot be applied. For initial value problems such as I want to describe here, the appropriate approach is provided by the functional integral formalism of the in-in generating functional for the Green functions of quarks and gluons, also referred to as closed-time-path (CTP) Green functions. The CPT formalism is a powerful Green function formulation, originally introduced by Schwinger [3] and Keldysh [4] for describing general nonequilibrium phenomena in field theory [5,7-9]. In combination with the so-called nonlocal source theory and the loop expansion techniques developed by de Dominicis and Martin [10] and Cornwall, Jackiw, and Tomboulis [11], one obtains generalized Dyson-Schwinger equations which incorporate the initial state correlations and provide a systematic treatment of the quantum correlations to any order in $\hbar$. Furthermore, it allows one to describe phase-transition phenomena and dynamical symmetry breaking, issues that I will not address here, but which are of central interest when studying the confinement dynamics, as intended to be presented elsewhere. In this section, I will first review the concept of the in-in generating functional and the effective action for the CTP Green functions, and then derive the dynamical equations of motion. For additional reading on these techniques I refer to the extensive review of Chou et al. [7] and to the instructive work of Calzetta and $\mathrm{Hu}$ [8].

\section{A. Preliminaries}

The starting point is the QCD Lagrangian given in terms of the gluon fields $A_{a}^{\mu}$ and the quark fields $\psi$ and $\bar{\psi}$ [which are vectors in flavor space, $\left.\psi \equiv\left(\psi_{u}, \psi_{d}, \ldots\right)\right]$ :

$$
\begin{aligned}
\mathcal{L}\left[A^{\mu}, \psi, \bar{\psi}\right]= & -\frac{1}{4} F_{\mu \nu, a} F_{a}^{\mu \nu}+\overline{\psi_{i}}\left[\left(i \gamma_{\mu} \partial^{\mu}-\hat{m}\right) \delta_{i j}\right. \\
& \left.-g_{s} \gamma_{\mu} A_{a}^{\mu} T_{a}^{i j}\right] \psi_{j}+\xi_{a}\left(A^{\mu}\right),
\end{aligned}
$$

where $F_{a}^{\mu \nu}=\partial^{\mu} A_{a}^{\nu}-\partial^{\nu} A_{a}^{\mu}+g_{s} f_{a b c} A_{b}^{\mu} A_{c}^{\nu}$ is the gluon fieldstrength tensor. The subscripts $a, b$, and $c$ label the color components of the gluon fields, and $g_{s}$ denotes the color charge related to $\alpha_{s}=g_{s}^{2} /(4 \pi)$. The $T_{a}$ are the generators of the $\mathrm{SU}(3)$ color group, satisfying $\left[T_{a}, T_{b}\right]=i f_{a b c} T_{c}$ with the structure constants $f_{a b c}$. The indices $i$ and $j$ label the color 
components of the quark fields and $\hat{m} \equiv \operatorname{diag}\left(m_{u}, m_{d}, \ldots\right)$. I will in the following exploit the fact that at high energies the quark current masses $m_{f}$ can be neglected, which corresponds to the chiral limit where they are exactly zero.

In general the Lagrangian equation (1) must also include the Faddeev-Popov ghosts as independent field degrees of freedom. However, I will work exclusively in a class of ghost-free gauges, namely, the so-called axial gauges, which are defined by the gauge condition [32]

$$
n_{\mu} A_{a}^{\mu}(x) \equiv n \cdot A_{a}=0,
$$

where $n^{\mu}$ is a constant four-vector in the $x^{0}-x^{3}$ plane near the forward light cone such that ${ }^{1} n^{2} \neq 0$. It may be parametrized, e.g., as $n^{\mu}=(a+b, 0,0, a-b)$, with the condition $n^{2}$ $=4 a b \ll 1$. The associated gauge-fixing term is denoted by a function $\xi_{a}\left(A^{\mu}\right)$ which I take as

$$
\xi_{a}\left(A^{\mu}\right)=-\frac{1}{2 \alpha n^{2}} \partial_{\lambda}\left(n \cdot A_{a}\right) \partial^{\lambda}\left(n \cdot A_{a}\right) .
$$

Here $\alpha$ is the gauge parameter that specifies the type of axial gauge. In particular, I will henceforth set $\alpha=1$, which is known as the planar gauge. In contrast to covariant gauges where $\xi_{a}(A)=-1 /(2 \alpha)\left(\partial \cdot A_{a}\right)^{2}$, the class of gauges [Eq. (3)] is well known to have a number of advantages [27,32]. First, the ghost fields decouple from the gluon field and drop out. Second, the so-called Gribov ambiguity is not present in this gauge. Third, the gluon propagator involves only the two physical transverse polarizations, which will simplify the analysis considerably. Furthermore, it allows for a rigorous resummation of the perturbative series at high energies in terms of the leading logarithmic contributions and consequently leads to a simple probabilistic description of the perturbative parton evolution within the (modified) leading log approximation (MLLA) [27,28,33] in QCD.

The classical action corresponding to Eq. (1) is represented as

$$
I\left[A^{\mu}, \psi, \bar{\psi}\right] \equiv I^{(0)}\left[A^{\mu}\right]+I^{(0)}[\psi, \bar{\psi}]+I^{(\mathrm{int})}\left[A^{\mu}, \psi, \bar{\psi}\right],
$$

where

$$
\begin{gathered}
I^{(0)}\left[A^{\mu}\right]=\int d^{4} x d^{4} y\left\{-\frac{1}{2} A_{a}^{\mu}(x)\left[D_{(0) \mu \nu}^{a b}(x, y)\right]^{-1} A_{b}^{\nu}(y)\right\}, \\
I^{(0)}[\psi, \bar{\psi}]=\int d^{4} x d^{4} y\left\{\bar{\psi}_{i}(x)\left[S_{(0)}^{i j}(x, y)\right]^{-1} \psi_{j}(y)\right\} \\
I^{(\mathrm{int})}\left[A^{\mu}, \psi, \bar{\psi}\right]=-\int d^{4} x\left\{g_{s} \gamma_{\mu} T_{a}^{i j} \overline{\psi_{i}}(x) A_{a}^{\mu}(x) \psi_{j}(x)\right. \\
+g_{s} f_{a b c}\left[\partial_{\mu} A_{\nu, a}(x)\right] A_{b}^{\mu}(x) A_{c}^{\nu}(x) \\
+g_{s}^{2} f_{a b c} f_{a b^{\prime} c^{\prime}} A_{\mu, b}(x) A_{\nu, c}(x) \\
\left.\times A_{b^{\prime}}^{\mu}(x) A_{c^{\prime}}^{\nu}(x)\right\},
\end{gathered}
$$

\footnotetext{
${ }^{1}$ One distinguishes further in "timelike" ("spacelike") axial gauge if $n^{2}>0\left(n^{2}<0\right)$, whereas the singular case $n^{2}=0$ is called the "light-cone", gauge.
}

with the kernels of the free parts $I^{(0)}\left[A^{\mu}\right]$ and $I^{(0)}[\psi, \bar{\psi}]$ given by

$$
\begin{gathered}
{\left[D_{(0) \mu \nu}^{a b}(x, y)\right]^{-1}=\delta_{a b} \delta^{4}(x-y) \square_{x}^{\mu \nu},} \\
{\left[S_{(0)}^{i j}(x, y)\right]^{-1}=\delta_{i j} \delta^{4}(x-y) i \gamma \cdot \partial_{x},}
\end{gathered}
$$

where the quark current masses are set to zero here and in the following. The operator $\square_{x}^{\mu \nu}$ is a generalized D'Alembertian containing the remnant of the gauge-fixing term of Eq. (1), which for the gauge Eq. (3) with $\alpha=1$ reads

$$
\square_{x}^{\mu \nu} \equiv\left(g^{\mu \nu}-\frac{n^{\mu} \partial_{x}^{\nu}+n^{\nu} \partial_{x}^{\mu}}{n \cdot \partial_{x}}\right) \square_{x}
$$

with $\square_{x}=\partial_{x} \cdot \partial_{x}$ and $\partial_{x}^{\mu}=\partial / \partial x^{\mu}$. The inverses of Eq. (6) are the free gluon and quark Feynman propagators, i.e., the expectation values of the time-ordered products of the noninteracting fields $\quad-i\left\langle T A_{\mu}(x) A_{\nu}(y)\right\rangle_{(0)} \quad$ and $-i\langle T \psi(x) \bar{\psi}(y)\rangle_{(0)}$,

$$
\begin{gathered}
D_{(0) \mu \nu}^{a b}(x, y)=\int \frac{d^{4} k}{(2 \pi)^{4}} e^{-i k \cdot(x-y)} \delta_{a b} \frac{-d_{\mu \nu}(k)}{k^{2}+i \epsilon}, \\
d_{\mu \nu}(k)=g_{\mu \nu}-\frac{n_{\mu} k_{\nu}+n_{\nu} k_{\mu}}{n \cdot k}, \\
S_{(0)}^{i j}(x, y)=\int \frac{d^{4} p}{(2 \pi)^{4}} e^{-i p \cdot(x-y)} \delta_{i j} \frac{1}{\gamma \cdot p+i \epsilon} .
\end{gathered}
$$

It is noteworthy that the form of the gluon propagator ${ }^{2}$ arises from the sum over the two transverse gluon polarizations, $d_{\mu \nu}(k)=\sum_{s=1,2} \varepsilon_{\mu}(k, s) \cdot \varepsilon_{\nu}^{*}(k, s)$, having the properties $[27,32]$

$$
d_{\mu}^{\mu}(k)=2, \quad k_{\mu} d^{\mu \nu}(k)=-\frac{n^{\nu} k^{2} k^{2} \rightarrow 0}{n \cdot k} \stackrel{\rightarrow}{\rightarrow} 0,
$$

meaning that only the two physical polarization states propagate, with $\varepsilon_{\mu} k^{\mu}=0$. For comparison, in the covariant Feynman gauge, $d^{\mu \nu}=g^{\mu \nu}, d_{\mu}^{\mu}=4$, and $k_{\mu} d^{\mu \nu}=k^{\nu} \neq 0$.

In going over from the classical action [Eq. (4)] to a quantum field formulation, the fields become Heisenberg operators. Let me introduce a compact notation for the different field degrees of freedom $f::^{3}$

$$
\begin{gathered}
\phi_{f}:=\left(A^{\mu}, \psi, \bar{\psi}\right)=\left(A^{\mu}, \psi_{u}, \bar{\psi}_{u}, \psi_{d}, \bar{\psi}_{d}, \ldots\right), \\
f=g, u, \bar{u}, d, \bar{d}, \ldots .
\end{gathered}
$$

The state of the system may be characterized by the Heisenberg field operator $\Phi_{H}(x)$, where $\Phi_{H} \equiv \Phi_{H}\left[\phi_{f}\right]$ and $x=(t, \vec{x})$. Its time evolution is determined by the Hamiltonian $H=H^{(0)}+H^{(\mathrm{int})}$ of the system $\left(\partial_{t} \equiv \partial / \partial t\right)$,

\footnotetext{
${ }^{2}$ The apparent singularity of $d_{\mu \nu}(k)$ at $n \cdot k \simeq k^{+}=0$ must be dealt with in the usual $i \epsilon$ prescription, or by taking the principal value.

${ }^{3}$ Since the quarks and antiquarks are treated as massless here, the different quark flavors are, with respect to the strong interaction, merely copies of each other.
} 


$$
\partial_{t} \Phi_{H}(x)=i\left[H, \Phi_{H}(x)\right]_{-} .
$$

Defining $t=t_{0}$ as the initial point for the time evolution of the system, the associated Heisenberg state vectors obey

$$
|\phi(t)\rangle=U_{J}\left(t, t_{0}\right)\left|\phi\left(t_{0}\right)\right\rangle,
$$

where

$$
U_{J}\left(t, t_{0}\right) \equiv T \exp \left[-i \int_{t_{0}}^{t} d t^{\prime} d^{3} x^{\prime} J\left(x^{\prime}\right) \Phi_{H}\left(x^{\prime}\right)\right] .
$$

$T$ denotes the usual time-ordering operator, and the external source $J$ is understood as a sum over sources for the various degrees of freedom. Note that the adjoint $U_{J}^{\dagger}\left(t, t_{0}\right)$ $=T^{\dagger} \exp \left[i \int_{t_{0}}^{t} d^{4} x^{\prime} J\left(x^{\prime}\right) \Phi_{H}\left(x^{\prime}\right)\right]$ involves an antitemporal ordering $T^{\dagger}$. In the absence of external sources, the state vectors are time independent: $|\phi(t)\rangle=\left|\phi\left(t_{0}\right)\right\rangle$.

Upon switching from the Heisenberg picture to the interaction picture, the time evolution of the corresponding interaction picture field $\Phi_{I}(x)$ is determined by the interaction Hamiltonian $H^{(\text {int })}$ alone,

$$
\partial_{t} \Phi_{I}(x)=i\left[H^{(\mathrm{int})}, \Phi_{I}(x)\right]_{-},
$$

where $\Phi_{I}$ is related to the Heisenberg $\Phi_{H}$ field by

$$
\Phi_{I}(x)=S\left(t, t_{0}\right) \Phi_{H}(x) S^{\dagger}\left(t, t_{0}\right),
$$

and evolves explicitly in time through

$$
S\left(t, t_{0}\right) \equiv T \exp \left[-i \int_{t_{0}}^{t} d t^{\prime} H^{(\mathrm{int})}\left(t^{\prime}\right)\right] .
$$

According to Eqs. (12) $-(16)$, at $t=t_{0}$ the Heisenberg picture and the interaction picture coincide, $\Phi_{H}\left(t_{0}, \vec{x}\right)$ $=\Phi_{I}\left(t_{0}, \vec{x}\right)$. Hence the interaction picture field $\Phi_{I}(x)$ can be expanded at $t_{0}$ in terms of a Fock basis of free particle states, the in basis,

$$
\begin{gathered}
\Phi_{I}(x)=\sum_{f=g, q, \bar{q}} \int \frac{d^{4} p}{(2 \pi)^{4}} \theta\left(p^{0}\right)(2 \pi) \delta\left(p^{2}\right) \\
\times \sum_{s}\left[e^{-i p \cdot x} a_{f}(p, s)+e^{i p \cdot x} a_{f}^{\dagger}(p, s)\right], \\
\left|n^{(1)}, n^{(2)}, \ldots, n^{(\infty)}\right\rangle=\prod_{f} \prod_{i} \frac{1}{\sqrt{n_{f}^{(i)} !}}\left[a_{f}^{\dagger}\left(p_{i}, s_{i}\right)\right]^{n_{f}^{(i)}}|0\rangle,
\end{gathered}
$$

where the $a_{f}^{\dagger}\left(a_{f}\right)$ are the corresponding creation (destruction) operators for the particle types $f=g, q, \bar{q}$ with definite momentum $p_{i}$ and spin $s_{i}$, the $n_{f}^{(i)}$ are the occupation numbers of the particle states, and

$$
a_{f}\left(p_{i}, s_{i}\right)|0\rangle=0, \quad n_{f}^{(i)}=\left\langle n_{f}^{(i)}\left|a_{f}\left(p_{i}, s_{i}\right) a_{f}^{\dagger}\left(p_{i}, s_{i}\right)\right| n_{f}^{(i)}\right\rangle .
$$

Thus a general multiparton state $|\phi\rangle$ at time $t_{0}$ is given by a superposition of such states:

$$
\left|\phi\left(t_{0}\right)\right\rangle=\sum_{n^{(i)}} C\left(n^{(1)}, n^{(2)}, \ldots, n^{(\infty)}\right)\left|n^{(1)}, n^{(2)}, \ldots, n^{(\infty)}\right\rangle,
$$

with scalar coefficients $C$. Alternatively, the initial state of the system at $t_{0}$ can be characterized by the statistical operator, or density matrix,

$$
\hat{\rho}\left(t_{0}\right)=\left|\phi\left(t_{0}\right)\right\rangle\left\langle\phi\left(t_{0}\right)\right|, \quad\left(\hat{\rho}_{0}\right)_{i j} \equiv\left\langle n^{(i)}\left|\hat{\rho}\left(t_{0}\right)\right| n^{(j)}\right\rangle,
$$

which in the Heisenberg representation is time independent, but in the interaction picture evolves with time according to

$$
\partial_{t} \hat{\rho}=i\left[H^{(\mathrm{int})}, \hat{\rho}\right]_{-},
$$

so that

$$
\hat{\rho}(t)=S^{\dagger}\left(t, t_{0}\right) \hat{\rho}\left(t_{0}\right) S\left(t_{0}, t\right),
$$

where $S$ is defined by Eq. (16). For instance, a general density matrix that describes any form of single-particle density distribution at $t_{0}$ is

$$
\begin{aligned}
\hat{\rho}\left(t_{0}\right)= & N \exp \left[\sum_{f, s} \int_{\Omega} d^{3} x \int \frac{d^{3} p}{(2 \pi)^{3} 2 p^{0}}\right. \\
& \left.\times F_{f}\left(t_{0}, \vec{x}, p\right) a_{f}^{\dagger}(p, s) a_{f}(p, s)\right],
\end{aligned}
$$

where $\Omega$ denotes the hypersurface of the initial values and $F_{f}$ is a $c$-number function related to the single-particle phase-space density of particle species $f$ at $\vec{x}$ with fourmomentum $p$, and $N$ is a normalization factor.

\section{B. The CTP generating functional}

After these preliminaries let me turn now to describe the time development of the multiparton state from the initial state $\left|\phi^{\text {in }}\right\rangle=\left|\phi\left(t_{0}\right)\right\rangle$, continuously through finite intermediate times $t_{0}<t<t_{\infty}$, to some final state $\left|\phi^{\text {out }}\right\rangle=\left|\phi\left(t_{\infty}\right)\right\rangle$ in the remote future (see Fig. 1). In the usual $S$-matrix formalism of quantum field theory one calculates the in-vacuum to out-vacuum amplitude $Z[J]=\left\langle 0^{\text {in }} \mid 0^{\text {out }}\right\rangle_{J}$, and from this, physical quantities corresponding to in-out $S$-matrix elements of certain operators, assuming that the Fock space of the asymptotic out states is the same as for the in states [Fig. 1(a)], as, e.g., in scattering theory. In the present case, however, the system evolves forward through finite points of time, and so the asymptotic out basis $\left|\phi^{\text {out }}\right\rangle$ is not known before the solution to the problem. There is an arrow of time, leading to an irreversible evolution. Moreover, in general $\left|0^{\text {in }}\right\rangle \neq\left|0^{\text {out }}\right\rangle$, as for instance in the case of a phase transition or spontaneous symmetry breaking where in and out vacua are of different natures.

These problems can be overcome by using the CTP formalism based on in-in rather than in-out matrix elements $[7,12]$, but otherwise using the familiar techniques of the path integral method for quantizing the theory. The in-in generating functional is defined as the in-vacuum to in-vacuum amplitude $Z[J, \hat{\rho}]=\operatorname{Tr} \Sigma_{\varphi}\left\langle 0^{\mathrm{in}} \mid \varphi\right\rangle_{J}\left\langle\varphi|\hat{\rho}| 0^{\mathrm{in}}\right\rangle_{J}$, including possible initial state correlations represented by the 
density matrix $\hat{\rho}$ at $t_{0}$, and a sum over a complete set of states $\varphi$ at $t_{\infty}$ [Fig. 1(b)]. With reference to Appendix A, where the relevant concepts are reviewed and applied to the case of QCD, I merely state here the resulting path integral representation for the in-in or CTP generating functional. It is given by the following path integral representation in twopoint source approximation:

$$
\begin{aligned}
Z_{P}\left[J^{\mu}, j, \bar{j}, K^{\mu \nu}, k\right]= & e^{i W_{P}\left[J^{\mu}, j, \grave{j}, K^{\mu \nu}, k\right]} \\
= & \int \mathcal{D} A_{\alpha}^{\mu} \mathcal{D} \psi_{\alpha} \mathcal{D} \bar{\psi}_{\alpha} \\
& \times \exp \left[i \left(I\left[A_{\alpha}^{\mu}, \psi_{\alpha}, \bar{\psi}_{\alpha}\right]+J_{\mu}^{\alpha} A_{\alpha}^{\mu}+j^{\alpha} \bar{\psi}_{\alpha}\right.\right. \\
& \left.\left.+\bar{j}^{\alpha} \psi_{\alpha}+\frac{1}{2} A_{\alpha}^{\mu} K_{\mu \nu}^{\alpha \beta} A_{\beta}^{\nu}+\bar{\psi}_{\alpha} k^{\alpha \beta} \psi_{\beta}\right)\right],
\end{aligned}
$$

where I introduced a shorthand notation for the integration over the space-time variables to be understood in the functional sense:

$$
\begin{gathered}
J \phi \equiv \int_{P} d^{4} x J(x) \phi(x), \\
\phi K \phi \equiv \int_{P} d^{4} x d^{4} y \phi(x) K(x, y) \phi(y) .
\end{gathered}
$$

The CTP generating functional [Eq. (25)] differs from the usual generating functional of QCD in two essential aspects.

First, it contains both local sources $(J, j, \bar{j})$ and nonlocal two-point sources $(K, k)$. The former represent not only the usual external source contribution $J_{\text {ext }}(x)$, but also the local source term $\widetilde{K}(x)$ for a possible dynamical background field present already at initial point $t=t_{0}$, that is, $J=J_{\text {ext }}+\widetilde{K}$, and similarly for $j$ and $\bar{j}$. The nonlocal sources $K(x, y)$ and $k(x, y)$, on the other hand, represent the two-particle initial state correlations at $t=t_{0}$. Both these source contributions ${ }^{4}$ stem from the general nontrivial density matrix $\hat{\rho}\left(t_{0}\right)$ that defines the initial ground state. In the usual field theory formulation both these source terms are absent. As a consequence, the connected generating functional $W_{P}=-i \ln Z_{P}$ in Eq. (25) gives both the nonlocal connected Green functions of gluons and quarks, including initial state correlations [denoted by $D_{\mu \nu}(x, y)$, respectively, $\left.S(x, y)\right]$, as well as possible local mean fields which physically can either arise through nonvanishing external sources, or, in the case of gluons, may be generated dynamically by the system itself depending on the initial conditions [denoted in the following by $\left.\widetilde{A_{\mu}}(x)\right]$.

Second, the CTP functional $Z_{P}$ is defined on a closed time path in the complex $t$ plane (indicated by the subscript $P$ ). This path $P$ for the time integration is illustrated in Fig. 2(a): the path goes forward from $t_{0}$ to $t_{\infty}$ on the positive branch,

\footnotetext{
${ }^{4}$ In the two-point sources approximation, the actually infinite series of nonlocal $n$-point sources that generate $n$-particle correlations is truncated beyond $n=2$ (cf. Appendix A).
}
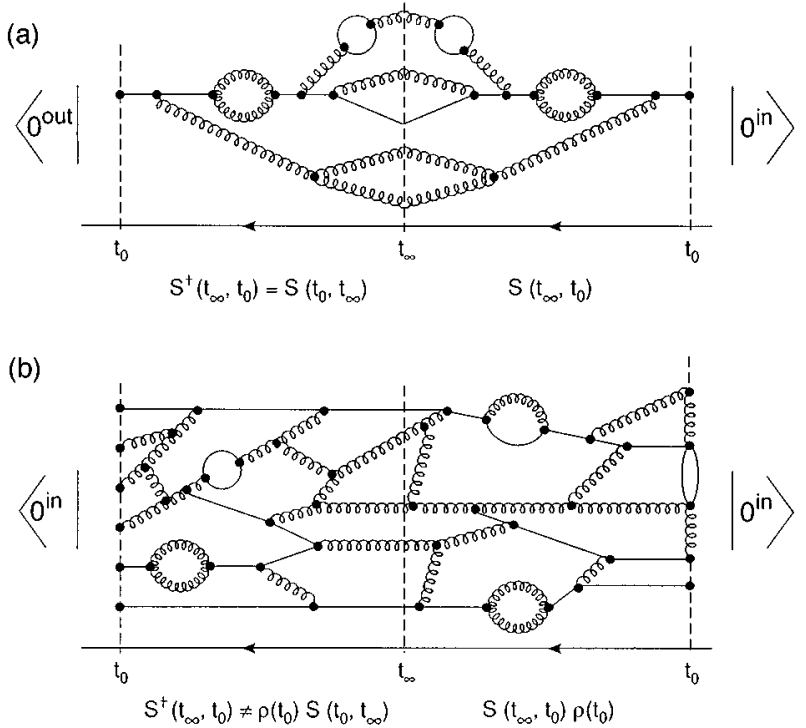

FIG. 1. Illustration of the difference of expectation values in the in-out and the in-in formalisms, corresponding to the time-ordered product of field operators. (a) In the usual $S$-matrix formalism with a trivial (diagonal) density matrix $\hat{\rho}\left(t_{0}\right)=\hat{1}$ and $\left|0^{\text {in }}\right\rangle=\left|0^{\text {out }}\right\rangle$, it suffices to calculate $\left\langle 0^{\text {out }}|\cdots| 0^{\text {in }}\right\rangle$, because of the symmetry of the time paths $\left(t_{0}, t_{\infty}\right)$ and $\left(t_{\infty}, t_{0}\right)$. (b) In the general case of a nontrivial initial state with multiparticle correlations described by $\hat{\rho}\left(t_{0}\right) \neq \hat{1}$, one must account for the complete time evolution on a closed time path from $t_{0}$ to $t_{\infty}$ and back to $t_{0}$ by calculating $\left\langle 0^{\text {in }}|\cdots \hat{\rho}| 0^{\text {in }}\right\rangle$.

and then back from $t_{\infty}$ to $t_{0}$ on the negative branch. Accordingly the generalized time-ordering $T_{P}$ is defined such that any point on the negative branch is understood at a later instant than any point on the positive branch. This is not merely a mathematical trick to restore analogy with the usual quantum field theory, but provides the means to compute expectation values for physical observables at finite time in contrast to the $S$-matrix formalism. The interpretation of this closed-time path is simple: although for physical observables the time values are on the positive branch, both positive and negative branches will come into play at intermediate steps in a self-consistent calculation, corresponding to a quantum mixing of positive and negative energy solutions. Therefore, in contrast to the usual path integral formulation of quantum field theory, the nonlocal two-point Green functions $D_{\mu \nu}(x, y)$ and $S(x, y)$ for gluons and quarks, respectively, each come in four different forms corresponding to the possible time orderings $\alpha \beta=++,+-,-+,--$ along the closed time path $P$, as illustrated in Fig. 2(b). In as much as the propagators $D_{\mu \nu}(x, y)$ and $S(x, y)$ can have values $x$ and $y$ on either the positive branch or the negative branch on the contour $P$, it is convenient to represent them $2 \times 2$ matrices $G(x, y) \equiv D_{\mu \nu}, S$ with components $G^{\alpha \beta}$ (a convention which holds for any two-point function defined along the closed time path $P$ ),

$$
\begin{aligned}
G(x, y)=-i\left\langle T_{P} \phi(x) \phi^{\dagger}(y)\right\rangle & \equiv\left(\begin{array}{ll}
G^{++} & G^{+-} \\
G^{-+} & G^{++}
\end{array}\right) \\
& \equiv\left(\begin{array}{cc}
G^{F} & G^{>} \\
G^{<} & G^{\bar{F}}
\end{array}\right),
\end{aligned}
$$


(a)

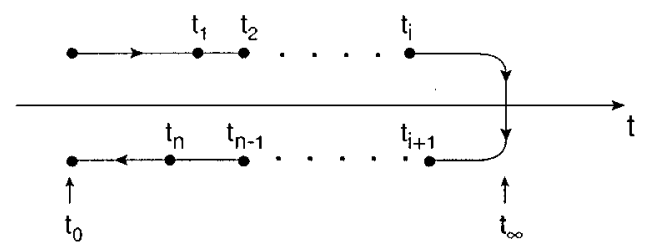

(b)
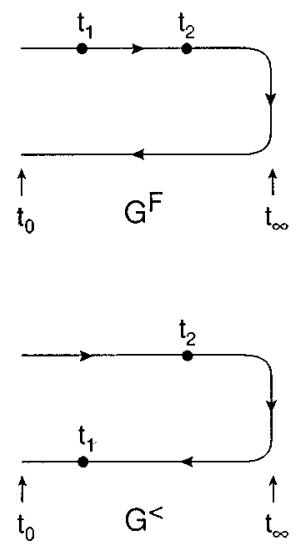

FIG. 2. (a) The closed time path in the complex $t$ plane for the evolution of operator expectation values in an arbitrary initial state. Any point on the forward, positive branch $t_{0} \rightarrow t_{\infty}$ is understood at an earlier instant than any point on the backward, negative branch $t_{\infty} \rightarrow t_{0}$. (b) The four different possible time orderings $\left(t_{1}, t_{2}\right)$ in the arguments of the two-point Green functions $G(x, y)$ $=G\left(t_{1}, \vec{x} ; t_{2}, \vec{y}\right)$, corresponding to $G^{F}, G^{>}, G^{<}, G^{\bar{F}}$.

where $\langle\cdots\rangle \equiv\left\langle\phi^{+}, t_{0}|\cdots| \phi^{-}, t_{0}\right\rangle$ denotes the vacuum expectation value, if $\left|\phi, t_{0}\right\rangle=|0\rangle$, or else the appropriate ensemble average. The generalized time-ordering operator $T_{P}$ is defined as

$T_{P} A(x) B(y):=\theta_{P}\left(x_{0}, y_{0}\right) A(x) B(y) \pm \theta_{P}\left(y_{0}, x_{0}\right) B(y) A(x)$,

where the $+(-)$ sign refers to boson (fermion) operators, and the $\theta$ function with two time arguments $x_{0}, y_{0}$ is defined on the closed time path $P$ (Fig. 2) as

$$
\theta_{P}\left(x_{0}, y_{0}\right)= \begin{cases}1 & \text { if } x_{0} \text { succeeds } y_{0} \text { on the contour } P \\ 0 & \text { if } x_{0} \text { precedes } y_{0} \text { on the contour } P\end{cases}
$$

Hence $T_{P}$ coincides with the usual temporal ordering $T$ on the positive branch $\left(t_{0} \rightarrow t_{\infty}\right)$ of the closed time path in Fig. 2 , but represents antitemporal ordering $T^{\dagger}$ on the negative branch $\left(t_{\infty} \rightarrow t_{0}\right)$. The notation on the right hand side expresses that $G^{F}$ is the usual Feynman causal propagator, $G^{\bar{F}}$ is the corresponding anticausal propagator, and $G^{>}$ $\left(G^{<}\right)$is the correlation function for $x_{0}>y_{0}\left(x_{0}<y_{0}\right)$. Explicitly, (a)

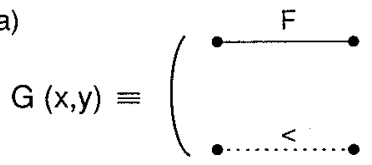

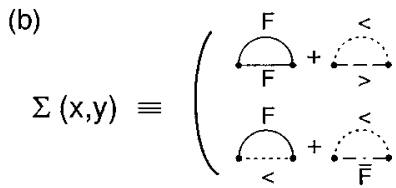
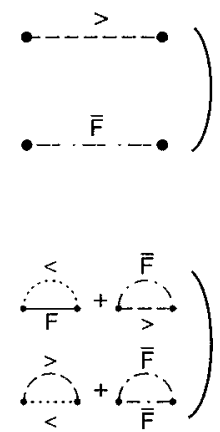

FIG. 3. Matrix representation of the CTP two-point functions: (a) The Green function $G(x, y)$, and (b) the self-energy function $\hat{\Sigma}(x, y) \propto[G(x, y)]^{2}$.

$$
\begin{gathered}
D_{\mu \nu}^{F}(x, y)=-i\left\langle T A_{\mu}(x) A_{\nu}(y)\right\rangle, \\
D_{\mu \nu}^{>}(x, y)=+i\left\langle A_{\nu}(y) A_{\mu}(x)\right\rangle, \\
D_{\mu \nu}^{<}(x, y)=-i\left\langle A_{\mu}(x) A_{\nu}(y)\right\rangle, \\
D_{\mu \nu}^{\bar{F}}(x, y)=-i\left\langle T^{\dagger} A_{\mu}(x) A_{\nu}(y)\right\rangle,
\end{gathered}
$$

and

$$
\begin{aligned}
& S^{F}(x, y)=-i\langle T \psi(x) \bar{\psi}(y)\rangle, \quad S^{>}(x, y)=-i\langle\bar{\psi}(y) \psi(x)\rangle, \\
& S^{<}(x, y)=-i\langle\psi(x) \bar{\psi}(y)\rangle, \quad S^{\bar{F}}(x, y)=-i\left\langle T^{\dagger} \psi(x) \bar{\psi}(y)\right\rangle .
\end{aligned}
$$

The CTP generating functional $Z_{P}=\exp \left(i W_{P}\right)$, Eq. (25), is the fundamental starting point for deriving the dynamical equations of motion for both the gluon mean field $\widetilde{A}^{\mu}$ and the dressed gluon and quark propagators $D^{\mu \nu}$ and $S$, using the matrix representations (27)-(31). Formally, this Green function formalism on the closed time path is completely analogous to the usual quantum field theory, except that all propagators, self-energies, etc., are now $2 \times 2$ matrices, as diagrammatically represented in Fig. 3. Correspondingly, the Feynman rules remain the same, but each propagator line of a Feynman diagram can be any of the four components of the Green functions.

\section{The CTP effective action}

To proceed, it is convenient to work with the CTP effective action $\Gamma_{P}$, the $t w o$-particle irreducible vertex functional, which determines the equations of motion for the physically relevant Green functions and the mean field, rather than with $Z_{P}$ or $W_{P}$ of Eq. (25) which involve the sources $J$ and $K$ that do not have any immediate physical interpretation. The CTP effective action $\Gamma_{P}$ is defined as the multiple Legendre transform of $W_{P}[7,8]$, which with respect to the two-point source representation (25) is given by

$$
\begin{aligned}
\Gamma_{P}\left[\widetilde{A^{\mu}}, D^{\mu \nu}, S\right]= & W_{P}\left[J^{\mu}, j, \bar{j}, K^{\mu \nu}, k\right]-\left(J_{\mu} \widetilde{A^{\mu}}+j \bar{\psi}+\bar{j} \psi\right) \\
& -\left(\frac{1}{2} A^{\mu} K_{\mu \nu} A^{\nu}-\bar{\psi} k \psi\right) .
\end{aligned}
$$

Note that $\Gamma_{P}$ reduces to the usual effective action for the one-particle irreducible vertex functions in the limit of vanishing mean field $\widetilde{A^{\mu}}=0$ and absence of initial state correlations $K=k=0$. In the general case, one obtains 


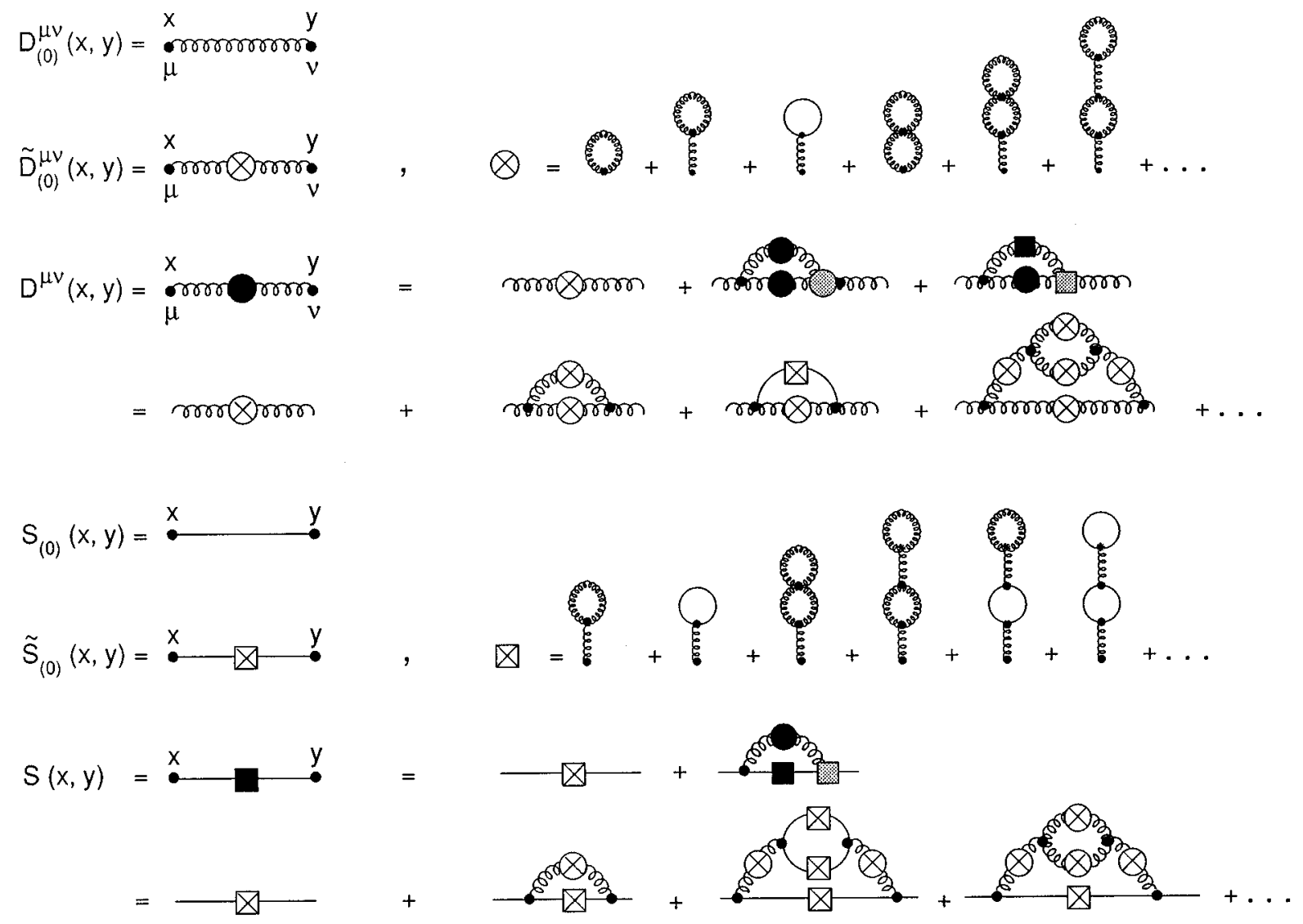

FIG. 4. Diagrammatic representation of the Green functions (i) $G_{(0)}(x, y)$ of the bare propagators, (ii) $\widetilde{G}_{(0)}(x, y)$, including the effect of a mean field by dressing the bare propagators with a dynamical mass, and (iii) $G(x, y)$, the full propagators, dressed by both local mean field and nonlocal quantum self-interactions (Dyson-Schwinger equations).

$$
\begin{aligned}
\Gamma_{P}\left[\widetilde{A}^{\mu}, D^{\mu \nu}, S\right]= & \widetilde{I}\left[\widetilde{A}^{\mu}\right]-\frac{i}{2} \operatorname{Tr}\left[\ln \left(D_{(0)}^{-1} D\right)-\widetilde{D}_{(0)}^{-1} D+1\right] \\
& +i \operatorname{Tr}\left[\ln \left(S_{(0)}^{-1} S\right)-\widetilde{S}_{(0)}^{-1} S+1\right] \\
& +\Gamma_{P}^{(2)}\left[\widetilde{A}^{\mu}, D^{\mu \nu}, S\right] .
\end{aligned}
$$

The first term is of order $\hbar^{0}$ and is given by the classical action (4) [and Eq. (A9) of Appendix A] with

$$
\left.\widetilde{I}\left[\widetilde{A}^{\mu}\right] \equiv I\left[A_{\alpha}^{\mu}, \psi_{\alpha}, \bar{\psi}_{\alpha}\right]\right|_{A_{\alpha}^{\mu}=\widetilde{A}^{\mu}, \psi_{\alpha}=\bar{\psi}_{\alpha}=0} .
$$

The second and third terms are of order $\hbar^{1}$ and correspond to the gluon and quark contributions in which the bare propagators $D_{(0)}^{\mu \nu}$ and $S_{(0)}(8)$ are modified by the presence of a local gluon mean field $\widetilde{A^{\mu}}$ leading to "mean-field-dressed" propagators $\widetilde{D}_{(0)}^{\mu \nu}$ and $\widetilde{S}_{(0)}$ with an effective screening mass $\widetilde{\mu} \equiv \widetilde{\mu}\left[\widetilde{A}^{\mu}\right]$ (see Fig. 4). In analogy with Eq. (6),

$$
\begin{gathered}
\left(\widetilde{D}_{(0)}^{-1}\right)^{\mu \nu}(x, y)=\left(D_{(0)}^{-1}\right)^{\mu \nu}(x, y)-\widetilde{\mu}_{g}^{\mu \nu}(x, y) \delta^{4}(x-y), \\
\widetilde{S}_{(0)}^{-1}(x, y)=S_{(0)}^{-1}(x, y)-\widetilde{\mu}_{q}(x, y) \delta^{4}(x-y) .
\end{gathered}
$$

Thus the effect of the mean field is to shift the pole in the bare Green functions [Eq. (6)] by a dynamical mass function $\tilde{\mu}$.

The last term $\Gamma_{P}^{(2)}$ in Eq. (33) represents the sum of all two-particle irreducible graphs of order $\hbar^{2}, \hbar^{3}, \ldots$ [11], with full propagators $D^{\mu \nu}$ and $S$, dressed by both local mean field and nonlocal self-interactions (see Fig. 4). As will become clear, the real (dispersive) part of $\Gamma_{P}^{(2)}$ contains the virtual loop corrections associated with the self-interactions of gluons and quarks, whereas the imaginary (dissipative) part contains the real emission, absorption, and scattering processes. In other words, $\Gamma_{P}^{(2)}$ embodies all the interesting quantum dynamics that is connected with renormalization group, entropy generation, dissipation, etc. Explicitly writing out the color indices, it is given by [see Fig. 5(a)]

$$
\begin{aligned}
\Gamma_{P}^{(2)}\left[\widetilde{A^{\mu}}, D^{\mu \nu}, S\right]= & -\frac{g_{s}^{2}}{2} \int d^{4} x d^{4} y \\
& \times \operatorname{Tr}\left[\int d^{4} z_{1} d^{4} z_{2} \lambda_{\mu \mu^{\prime} \mu^{\prime \prime}}^{a a^{\prime} a^{\prime \prime}} \Lambda_{\nu^{\prime \prime} \nu^{\prime} \nu}^{b^{\prime \prime} b^{\prime} b}\left(z_{2}, z_{1} ; y\right)\right. \\
& \times D_{a^{\prime} b^{\prime}}^{\mu^{\prime} \nu^{\prime}}\left(x, z_{1}\right) D_{a^{\prime \prime} b^{\prime \prime}}^{\mu^{\prime \prime} \nu^{\prime \prime}}\left(x, z_{2}\right) D_{b a}^{\nu \mu}(y, x) \\
& +\iint d^{4} z_{1} d^{4} z_{2} \gamma_{\mu} T_{i i^{\prime}}^{a} \Xi_{j j^{\prime} \nu}^{b}\left(z_{2}, z_{1} ; y\right) \\
& \left.\times D_{a b}^{\mu \nu}\left(x, z_{1}\right) S_{i^{\prime} j^{\prime}}\left(x, z_{2}\right) S_{i j}(y, x)\right]
\end{aligned}
$$

Here $\Lambda_{\nu \nu^{\prime} \nu^{\prime \prime}}$ and $\Xi_{\nu}$ are the $g g g$ and $q q g$ vertex functions, respectively, 
(a)

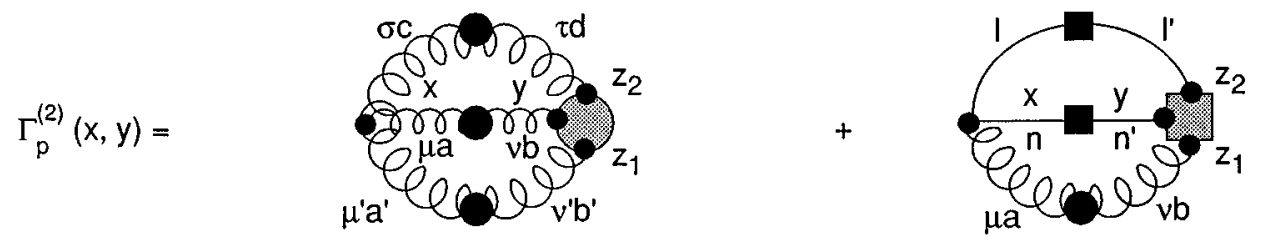

(b)
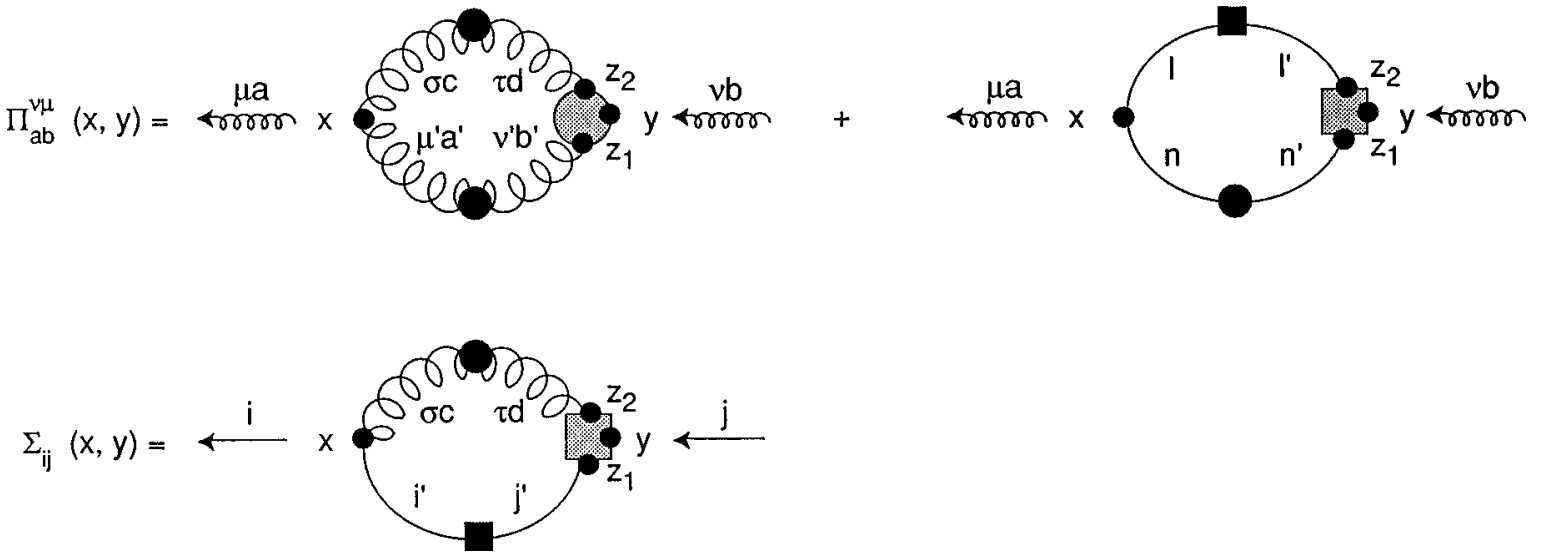

FIG. 5. (a) Diagram of the function $\Gamma_{P}^{(2)}$, Eq. (36), representing the sum of all two-particle irreducible graphs of order $\hbar^{2}, \hbar^{3}, \ldots$, with fully dressed propagators $D^{\mu \nu}$ and $S$. (b) Illustration of the self-energies $\Pi^{\mu \nu}$ and $\Sigma$, Eqs. (42) and (43), which derive from $\Gamma_{P}^{(2)}$ by functional differentiation with respect to $D^{\mu \nu}$ and $S$.

$$
\begin{aligned}
\Lambda_{\nu \nu^{\prime} \nu^{\prime \prime}}^{a a^{\prime} a^{\prime \prime}}\left(z_{1}, z_{2} ; y\right)= & \lambda_{\nu \nu^{\prime} \nu^{\prime \prime}}^{a a^{\prime} a^{\prime \prime}} \delta^{4}\left(y-z_{1}\right) \delta^{4}\left(y-z_{2}\right) g\left[\widetilde{A}^{\mu}(y)\right] \\
& +O\left(g_{s}^{2}\right) \\
\Xi_{i j \nu}^{a}\left(z_{1}, z_{2} ; y\right)= & \gamma_{\nu} T_{a}^{i j} \delta^{4}\left(y-z_{1}\right) \delta^{4}\left(y-z_{2}\right) g\left[\widetilde{A}^{\mu}(y)\right] \\
& +O\left(g_{s}^{2}\right)
\end{aligned}
$$

with $\lambda_{\nu \nu^{\prime} \nu^{\prime \prime}}^{a a^{\prime} a^{\prime \prime}}$ and $\gamma_{\mu} T_{a}^{i j}$ the corresponding bare vertices, and the function $g\left[\widetilde{A}^{\mu}\right]$ describes the effect due to the presence of the gluon mean field $\widetilde{A}^{\mu}$ as compared to free space, where $g[0]=1$.

\section{The self-consistent equations of motion}

The dynamical equations of motion for the gluon mean field and the gluon and quark Green functions in the absence of external sources are now as usual obtained from variation of the effective action $\Gamma_{P}$ with respect to its variables, and setting the external sources to zero. Hence by functional differentiation of $\Gamma_{P}$ in Eq. (33) with respect to the gluon mean field $\widetilde{A^{\mu}}$ one gets the Ginzburg-Landau equation [34]

$$
\begin{aligned}
\frac{\delta \Gamma_{P}}{\delta \widetilde{A}^{\mu}(x)}= & \frac{\delta \widetilde{I}\left[\widetilde{A}^{\mu}\right]}{\delta \widetilde{A}^{\mu}(x)}+2 i \operatorname{Tr}\left\{\frac{\delta\left[\widetilde{D}_{(0)}^{\mu \nu}\right]^{-1}}{\delta \widetilde{A}^{\mu}(x)}-\frac{1}{2} \frac{\delta\left[\widetilde{S}_{(0)}\right]^{-1}}{\delta \widetilde{A}^{\mu}(x)}\right\} \\
& +\frac{\delta \Gamma_{P}^{(2)}}{\delta \widetilde{A}^{\mu}(x)}=0
\end{aligned}
$$

Similarly, the variation of $\Gamma_{P}$ with respect to the dressed propagators $D^{\mu \nu}$ and $S$ gives the CTP version of the DysonSchwinger equations [35]:

$$
\begin{gathered}
i \frac{\delta \Gamma_{P}}{\delta D^{\mu \nu}(y, x)}=D_{\mu \nu}^{-1}(x, y)-\widetilde{D}_{(0) \mu \nu}^{-1}(x, y)+\Pi_{\mu \nu}(x, y)=0, \\
-i \frac{\delta \Gamma_{P}}{\delta S(y, x)}=S^{-1}(x, y)-\widetilde{S}_{(0)}^{-1}(x, y)+\Sigma(x, y)=0 .
\end{gathered}
$$

Here $\Pi$ and $\Sigma$ are $2 \times 2$ matrices analogous to Eq. (27), representing the proper self-energy parts of gluons and quarks. They are obtained by functional differentiation of the quantum contribution $\Gamma_{P}^{(2)}$ to the effective action [Eq. (33)]:

$$
2 i \frac{\delta \Gamma_{P}^{(2)}}{\delta D_{\nu \mu}^{b a}(y, x)}=\Pi_{a b}^{\mu \nu}(x, y), \quad-i \frac{\delta \Gamma_{L}^{(2)}}{\delta S_{i j}(y, x)}=\Sigma_{i j}(x, y) .
$$

From Eq. (36), one gets [cf. Fig. 5(b)],

$$
\begin{aligned}
\Pi_{a b}^{\mu \nu}(x, y)= & -i g_{s}^{2}\left[\int d^{4} z_{1} d^{4} z_{2} \lambda_{a a^{\prime} a^{\prime \prime}}^{\mu \mu^{\prime} \mu^{\prime \prime}} \Lambda_{\nu^{\prime \prime} \nu^{\prime} \nu^{\prime \prime} b^{\prime} b}^{b^{\prime \prime}}\left(z_{2}, z_{1} ; y\right)\right. \\
& \times D_{a^{\prime} b^{\prime}}^{\mu^{\prime} \nu^{\prime}}\left(x, z_{1}\right) D_{a^{\prime \prime} b^{\prime \prime}}^{\mu^{\prime \prime} \nu^{\prime \prime}}\left(z_{2}, x\right) \\
& +\int d^{4} z_{1} d^{4} z_{2} \gamma_{\mu} T_{i i^{\prime}}^{a} \Xi_{j j^{\prime} \nu^{\prime}}^{b}\left(z_{2}, z_{1} ; y\right) \\
& \left.\times S_{i j}\left(x, z_{1}\right) S_{i^{\prime} j^{\prime}}\left(x, z_{2}\right)\right]
\end{aligned}
$$$$
\Sigma_{i j}(x, y)=+i g_{s}^{2} \int d^{4} z_{1} d^{4} z_{2} \gamma_{\mu} T_{i i^{\prime}}^{a} \Xi_{j j^{\prime} \nu}^{b}\left(z_{2}, z_{1} ; y\right)
$$$$
\times D_{a b}^{\mu \nu}\left(x, z_{1}\right) S_{i^{\prime} j^{\prime}}\left(x, z_{2}\right),
$$ 
with the $g g g$ and $q q g$ vertex functions $\Lambda_{\mu \nu \tau}^{a b c}$ and $\Xi_{i j \mu}^{a}$, respectively, defined by Eq. (37).

The Dyson-Schwinger equations (39) and (40) can be brought into a more familiar form by employing the expressions for the free propagators [Eq. (6)]:

$$
\begin{aligned}
& {\left[\vec{\square}_{x, \mu \rho}+\tilde{\mu}_{g}^{2}(x, y)\right] D_{a b}^{\rho \nu}(x, y)} \\
& \quad=\delta_{a b} g^{\mu \nu} \delta_{P}^{4}(x, y)-\int_{P} d^{4} x^{\prime} \Pi_{\sigma, a, b^{\prime}}^{\mu}\left(x, x^{\prime}\right) D_{b^{\prime} b}^{\sigma \nu}\left(x^{\prime}, y\right), \\
& D_{a b}^{\rho \nu}(x, y)\left[\overleftarrow{\square}_{y, \mu \rho}+\tilde{\mu}_{g}^{2}(x, y)\right] \\
& \quad=\delta_{a b} g_{\mu \nu} \delta_{P}^{4}(x, y)-\int_{P} d^{4} x^{\prime} D_{\sigma, a, b^{\prime}}^{\mu}\left(x, x^{\prime}\right) \Pi_{b^{\prime} b}^{\sigma \nu}\left(x^{\prime}, y\right),
\end{aligned}
$$

and

$$
\begin{aligned}
& {\left[i \gamma \cdot \vec{\partial}_{x}-\tilde{\mu}_{q}(x, y)\right] S_{i j}(x, y)} \\
& \quad=\delta_{i j} \delta_{P}^{4}(x, y)+\int_{P} d^{4} x^{\prime} \Sigma_{i k}\left(x, x^{\prime}\right) S_{k j}\left(x^{\prime}, y\right), \\
& S_{i j}(x, y)\left[-i \gamma \cdot \grave{\partial}_{y}-\tilde{\mu}_{q}(x, y)\right] \\
& \quad=\delta_{i j} \delta_{P}^{4}(x, y)+\int_{P} d^{4} x^{\prime} S_{i k}\left(x, x^{\prime}\right) \Sigma_{k j}\left(x^{\prime}, y\right),
\end{aligned}
$$

where $\partial_{x}^{\mu}=\partial / \partial x^{\mu}, \square_{x}^{\mu \nu}$ is defined by Eq. (7), the time integrations on the right hand sides are understood along the contour $P$, and the generalized $\delta_{P}$ function is defined on the closed time path $P$ (Fig. 2) as

$$
\begin{aligned}
& \delta_{P}^{4}(x, y) \\
& \quad:= \begin{cases}+\delta^{4}(x-y) & \text { if } x_{0} \text { and } y_{0} \text { from positive branch, } \\
-\delta^{4}(x-y) & \text { if } x_{0} \text { and } y_{0} \text { from negative branch, } \\
0 & \text { otherwise. }\end{cases}
\end{aligned}
$$

Let me emphasize once more the essential difference from the usual quantum field theory: Eqs. (44) and (45) are matrix equations and represent four equations, one for each of the four correlators in Eqs. (30) [(31)]. In the limiting case where correlations among different partons vanish, one has $G^{>}=G^{<}=0$, and because $G^{F}=G^{\bar{F} \dagger}$, one recovers the standard Dyson-Schwinger equations in terms of the Feynman propagators alone. The first equation in Eqs. (44) [(45)], describes the change of the propagators in the argument $x$, whereas the second equation describes the change in $y$ of the adjoint propagators (adjoint $\dagger$ means Hermitian conjugate with simultaneous exchange of the arguments). A diagrammatic representation of these Dyson-Schwinger equations for the fully dressed Green functions $D_{\mu \nu}(x, y)$ and $S(x, y)$ is shown in the previous Fig. 4.

Let me summarize the considerations of this section. The CTP generating functional $Z_{P}$ involving initial state correlations of the form Eq. (21), described by the density matrix $\hat{\rho}$ at $t=t_{0}$, yields an infinite hierarchy of $n$-point Green functions, defined along the closed time path. As explained in
Appendix A, the truncation of this hierarchy beyond $n>2$ assumes that the dynamics may be described to sufficient accuracy by a possible local gluon mean field and the nonlocal two-point Green functions of gluons and quarks, and that higher-order correlators are negligible. The resulting CTP effective action may then be represented by a systematic loop expansion corresponding to an expansion in powers of $\hbar$. Considering the pure quantum regime with zero mean field yields a coupled set of equations of motion for the gluon and quark propagators, which are $2 \times 2$ matrices containing the four possible time orderings of their arguments $x$ and $y$. The solution of these dynamical equations then boils down to the evaluation of expectation values involving the propagators and vertex functions, e.g., by using perturbation theory $[8,11]$.

\section{QUANTUM KINETIC THEORY}

Within the two-point source approximation to the full theory in terms of two-point Green functions, the resulting CTP Dyson-Schwinger equations (44) and (45) contain the quantum dynamics in terms of the dressed gluon and quark propagators $D_{\mu \nu}$ and $S$. Even with the neglect of higherorder correlators, the equations of motion are nonlinear, nonlocal integro-differential equations, generally not solvable in closed form. To make progress, one needs to supply reasonable physical input that allows one to make realistic approximations for multiparton systems of interest.

First of all, I will confine myself for the remainder of the paper to the pure quantum dynamics of gluons and quarks, when a gluon mean field is absent. That is, I choose the homogeneous initial condition $\widetilde{A^{\mu}}(x)=0$ at $t_{0}$, which in the absence of external sources implies that ${\widetilde{A^{\mu}}}^{\mu}$ will remain zero at all times $t>t_{0}$ :

$$
\frac{\delta \Gamma_{P}}{\delta \widetilde{A}^{\mu}(x)}=0, \quad \widetilde{A^{\mu}}(x)=0 .
$$

Consequently, in Eq. (33), the classical part $\widetilde{I}\left[\widetilde{A}^{\mu}\right]=0$ [see also Eq. (A4) of Appendix A], and $\widetilde{\mu}_{g}=\widetilde{\mu}_{q}=0$, so that the mean-field propagators reduce to the bare propagators:

$$
\widetilde{D}_{(0)}^{\mu \nu}(x, y)=D_{(0)}^{\mu \nu}(x, y), \quad \widetilde{S}_{(0)}^{\mu \nu}(x, y)=S_{(0)}(x, y) .
$$

This step, however, is not an approximation, but merely serves as a simplification in order not to overburden the following analysis. The more general case including a dynamical gluon background field causes in principle no severe additional complexities, and will be addressed elsewhere.

The essential approximation now is based on the "twoscale" nature of high-energy QCD, as mentioned in the Introduction. The dynamical evolution of a multiparton system can-in a reference frame where the partons move with highly relativistic velocities - be characterized by two different time (or length) scales, separated by time dilation and Lorentz contraction effects: a quantum field theoretical scale $\Delta r_{\text {qua }}$ and a statistical-kinetic scale $\Delta r_{\text {kin }}$. This is illustrated in Fig. 6(a). The quantum length scale $\Delta r_{\text {qua }}$, measures the spatial range of quantum fluctuations associated with the partons' self-interactions, and thus specifies the Compton wavelength $\lambda_{c} \equiv \mu_{g q}^{-1}$ of dressed partons. These gluon emission 
(a)

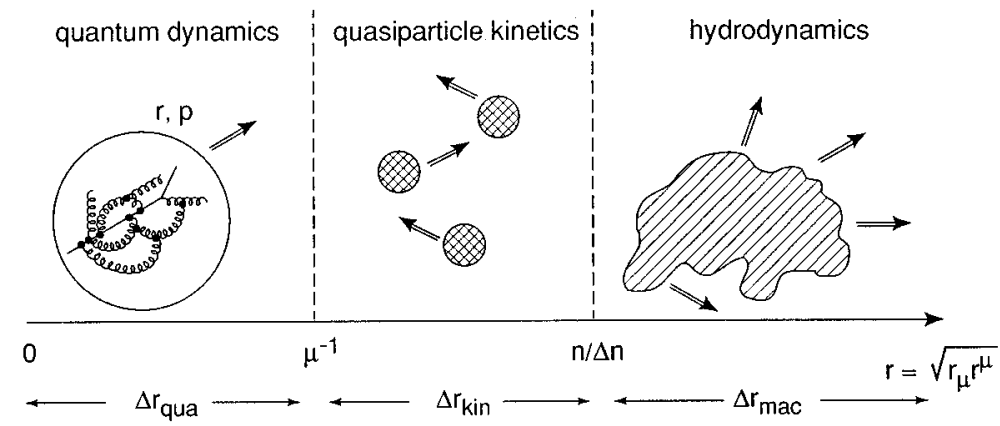

FIG. 6. (a) Classification of the different scales of relevance: (i) the quantum scale $\Delta r_{\text {qua }}$, of the order of the spatial extent of quantum fluctuations associated with the "radiative", self-energies, and defining a dressed parton state as a quasiparticle; (ii) the kinetic scale $\Delta r_{\text {kin }}$, measuring the range of correlations and binary interactions between these quasiparticles, with the "collisional" self-energies; and (iii) the "'macroscopic', scale $\Delta r_{\text {mac }}$, where the dynamics can be described in terms of bulk thermodynamic variables or hydrodynamics. (b) The quality of separation of quantum and kinetic scales is controlled by the choice of $\mu(r)$. Because the multiparticle dynamics of the system in general may change the scale of separation in space-time, one may choose $\mu(r)$ variable to optimize the kinetic description.

and absorption processes, embodied in the self-energy, dress up the bare propagators and allow one to describe partons as quasiparticles with finite spatial extent, but with a dynamical substructure. This is nothing but the underlying philosophy of the usual parton description in QCD. The kinetic length scale $\Delta r_{\text {kin }}$, on the other hand, measures the range of binary interactions between these quasiparticles. These scattering processes may be described on a semiclassical level, provided the local density of the quasiparticles is smaller than a critical density where the particles begin to overlap and the separation between quantum and classical regimes breaks down. Quantitatively, one has to require that the mean free path $\lambda_{\mathrm{mf}}$ of particles is large compared to the radiative corrections to the Compton wavelength $\lambda_{c}$. The crucial point is that with increasing energy scale the latter range becomes increasingly short range, concentrated around the light cone [see Fig. 6(b)]. Hence, in most physical situations at high energies, the quantum and the kinetic scales separate to very good approximation, and in the asymptotic limit exactly. It is important to stress that both quantum and kinetic scales define the microscopic regime of a semiclassical particle description. It is to be distinguished from the macroscopic domain of the dynamics of the bulk parton matter, characterized by comparably large space-time distances of the order $n^{-1 / 3}$, or $n /\left(\partial_{r} n\right)$, where $n(r)$ is the density of quasiparticles. In this regime the system may be described by, e.g., hydrodynamical evolution, which is, however, beyond the scope of this paper.

To exemplify this concept, consider the simple case of a parton in a Lorentz frame in which it moves with large momentum $k^{+} \equiv E+k_{z}\left(k^{+2} \gg k^{2} \gg 1 \mathrm{GeV}^{2}\right)$ nearly with the speed of light along the forward light cone $x^{+} \equiv t+z$. The quantum fluctuations around this parton's classical trajectory stem from its self-interaction with the gluon radiation field, corresponding to gluon emissions and reabsorptions, that smear out its energy over an interval $\Delta E \sim k^{2} / k^{+}$. It may thus be pictured as an unstable particle with a typical lifetime $\Delta \tau_{p} \sim 1 / \Delta E$. On the one hand, in the direction parallel to the light cone, the parton's intrinsic fluctuations decouple from the soft vacuum fluctuations with $\Delta \tau_{v} \sim 1 / k^{+} \ll \Delta \tau_{p} \sim k^{+} / k^{2}$ [36]. On the other hand, in the transverse $x_{\perp}$ direction, the partonic fluctuations have a small spatial extent of $\Delta r_{\perp} \sim 1 / k_{\perp} \ll 1 \quad \mathrm{GeV}^{-1}$. Therefore, on kinetic scales $\Delta_{\text {kin }}>\mu^{-1}$, the parton appears as a dressed particle which can be considered quasiclassically as an extended object with a small transverse size $\Delta r_{\perp}$ and a comparably long lifetime $\Delta \tau_{p}$-a quasiparticle. On quantum scales, however, the dressed parton has a substructure, determined by its surrounding cloud of gluons which it emits and reabsorbs due to its quantum nature.

In this spirit I will classify the parton dynamics with respect to elementary and quasiparticle excitations, referring to them by the terms bare and dressed partons, respectively: (i) Bare partons are to be understood as pointlike, massless quanta in the absence of radiative self-interactions, i.e., before renormalization; (ii) dressed partons, on the other hand, are dressed by the quantum self-interactions with their radiation field, which renormalize their masses and couplings.

In the field theoretical parton language, a dressed parton with its dynamically generated renormalized mass can be described (in a frame where it moves close to the speed of light) as a bare quantum which is surrounded by a virtual cloud of other bare gluons and quark-antiquark pairs with which it emits and absorbs. Hence, on kinetic space-time scales, a dressed parton can be visualized as a quasiparticle, i.e., an extended object with a dynamical substructure that is determined by the short-distance quantum fluctuations.

\section{A. Definition of quantum and kinetic space-time scales}

The realization of the two space-time scales, shortdistance quantum and quasiclassical kinetic, allows one to 
reformulate the quantum field theoretical problem as a relativistic many-body problem within kinetic theory. The key element is to establish the connection between the quantum theoretical Green functions and the kinetic particle description in terms of parton phase-space densities. In particular, the aim is to describe the evolution of a multiparton ensemble, given at time $t_{0}$, with a certain spatial and a momentum distribution, by exploiting the two-scale nature of highenergy QCD. As explained before, this requires a choice of Lorentz frame, in which the quanta move very fast and the typical momentum scale of their binary interactions and associated radiative processes is sufficiently large such that the corresponding interaction times are small compared to the mean free time between mutual collisions. For example, imagine a high-energy reaction has produced an initial configuration of materialized partons (e.g., a hadronic or nuclear collision with $\sqrt{s} \gtrsim 100 \mathrm{GeV}$ per hadron). If $t_{0}$ denotes the earliest point of time in the lab frame, when the parton densities have evolved to satisfy $\Delta p \Delta r \gg 1$, where $1 / \Delta p$ measures the scale of the partons' intrinsic quantum motion and $\Delta r$ the space-time variation of the system of partons, then, for times $t>t_{0}$, an approximate incoherent treatment of quantum dynamics and kinetic evolution is justified, as has been shown by McLerran and Venugopalan [25].

With this physical scenario in mind, now suppose that space-time is discretized into cells, with their size chosen intermediate between quantum and kinetic scales such that the separation between the two scales is optimal [8]. Then the correlation between different cells will be negligible, and only when two space-time points corresponding to the arguments of the propagators or self-energies lie in the same cell will the two-point correlation contribute (as explained in Appendix B). Consequently, in a given cell, one can by construction neglect spatial inhomogeneities of the local gluon and quark densities of the multiparton system. Within each cell, one may therefore describe the short-distance quantum dynamics analogously as in vacuum or homogenous media, whereas inhomogeneities of the spatial parton distribution and relaxation phenomena associated with binary collisions become apparent as one moves from cell to cell. In continuous space-time, corrections to this discretized picture can be taken into account by a systematic expansion in terms of gradients of the spatial inhomogeneities of the parton distributions [Eq. (66) below].

In order to quantify this concept, let me clearly specify quantum and kinetic domains with respect to the cellular space-time. It is important to realize that both quantum and kinetic scales are of dynamical, "internal" nature, i.e., determined by the multiparton evolution itself. The classification of the two scales only makes sense in the presence of self- and mutual interactions. However, the class of highenergy parton systems addressed here is characterized by two corresponding "external" scales: first, the large energy scale of the reaction that produces the initial system of large momentum partons, and second, the initial local density of partons in phase space that depends on the type of reaction. The first property implies a large characteristic momentum transfer $q_{\perp}^{2} \equiv\left(k_{1}-k_{1}^{\prime}\right)^{2}$ of scattering processes $\left(k_{1} k_{2} \rightarrow k_{1}^{\prime} k_{2}^{\prime}\right)$ and radiative processes $\left(k_{1} \rightarrow k_{1}^{\prime} k_{2}^{\prime}\right)$. The second property, on the other hand, is related to the mean free path $\lambda_{\mathrm{mf}}$ and mean free time $\tau_{\mathrm{mf}}$ of partons in between subsequent scatterings. If the latter are large compared to the typical space-time extent $1 / q_{\perp}$ of the scattering and radiation processes, then an incoherent treatment of the binary collisions among partons, and of the partons' propagation with associated quantum fluctuations, is applicable. This condition may be characterized by the invariant mass scale $\mu_{g q}$, defined such that

$$
q_{\perp}^{2}>\mu_{g q}^{2}>\lambda_{\mathrm{mf}}^{-2} .
$$

The parameter $\mu_{g q}^{2}$ can be interpreted as defining the minimum virtuality of a dressed parton, or, correspondingly, its maximum size, or Compton wavelength $\lambda_{c}=\mu_{g q}^{-1}$, such that the applicability condition of the parton description is ensured. Consequently, the size of each space-time cell must be chosen large enough that the spread of the dressed partons' intrinsic quantum motion is localized inside its fourdimensional volume, but smaller than the mean free path of dressed partons in between scatterings. Accordingly, I define the cell size $\Delta r^{\mu} \equiv \Delta r^{0} \Delta^{3} r$ by

$$
\mu_{g q}^{-4}<\Delta r^{\mu} \equiv \mu^{-4}(r) \ll \Lambda_{\mathrm{QCD}}^{-4},
$$

where $\Lambda_{\mathrm{QCD}} \simeq 0.25 \mathrm{GeV}$ is the QCD renormalization scale. For example, a cell size $\Delta r \lesssim 0.1 \mathrm{fm}$ allows one to resolve particles with energy-momentum $\geqslant 2 \mathrm{GeV}$. One can then characterize the kinetic space-time evolution of the system by a velocity profile of cells $i$, located around the points $r_{i}^{\mu}$, with a four-dimensional cell volume in its rest frame:

$$
\Omega\left(r_{i}\right)=\int_{r^{\prime} \in \Omega} d^{4} r^{\prime}=\int_{r_{i}^{0}-\Delta r^{0} / 2}^{r_{i}^{0}+\Delta r^{0} / 2} d r^{0} \int_{\vec{r}_{i}-\Delta \vec{r} / 2}^{\vec{r}_{i}+\Delta \vec{r} / 2} d^{3} r^{\prime} \simeq \mu^{-4} .
$$

Each cell carries a total momentum

$$
P^{\mu}\left(r_{i}\right):=\left.\sum_{j=1}^{N_{g q}} k_{j}^{\mu}\right|_{\left(r_{j}^{0}, \vec{r}_{j}\right) \in \Omega\left(r_{i}\right)}
$$

and a total invariant virtuality (the incoherent sum of parton virtualities)

$$
Q^{2}\left(r_{i}\right):=\left.\sum_{j=1}^{N_{g q}} k_{j}^{2}\right|_{\left(r_{j}^{0}, \vec{r}_{j}\right) \in \Omega\left(r_{i}\right)},
$$

where the sums are over all dressed partons $j$ inside the cell $i$, i.e., those that are during a time slice $\Delta r^{0}=\mu^{-1}$ contained within $\Delta^{3} r=\mu^{-3}$ around space-time point $r_{i}$. The corresponding local four-flow velocity is $u^{\mu}\left(r_{i}\right)=P^{\mu} / P^{0}$. This cellular space-time picture is illustrated in Fig. 7(a).

The validity of the above cell picture is controlled by the condition that the different scales are well separated:

$$
P^{+2}\left(r_{i}\right) \gg Q^{2}\left(r_{i}\right) \geqslant \mu^{2}\left(r_{i}\right) \gg \Lambda_{\mathrm{QCD}}^{2},
$$

where $\quad P^{\mu}=\left(P^{+}, P^{-}, \vec{P}_{\perp}\right), \quad P^{ \pm}=P_{0} \pm P_{3}, \quad$ and $\quad P_{\perp}$ $=\sqrt{P_{1}^{2}+P_{2}^{2}}$ with $P^{+}\left(P^{-}\right)$the lightcone momentum (energy), $P^{2}=P^{+} P^{-}-P_{\perp}^{2}$, and the normalization of a cell state $|P\rangle$ is $\left\langle P \mid P^{\prime}\right\rangle=2 P^{+}(2 \pi)^{3} \delta^{3}\left(\vec{P}-\vec{P}^{\prime}\right)$. On the basis of Eq. (54) and in terms of these light-cone variables, the fourmomentum of a parton $j$ can be characterized by only two 
(a)

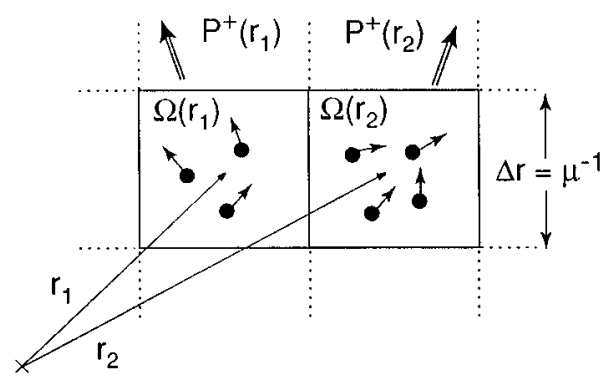

(b)

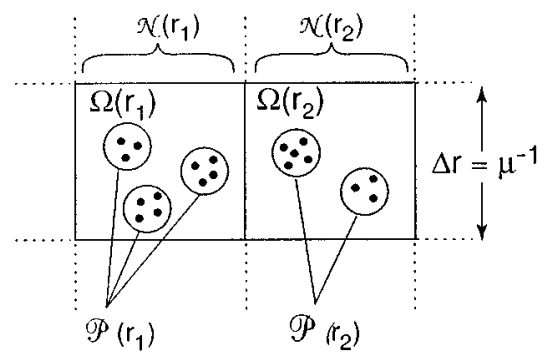

FIG. 7. (a) Illustration of the cellular space-time picture, with cell size chosen intermediate between quantum and kinetic scales such that the separation between the two scales is optimal, so that short-distance quantum correlation between different cells is negligible. (b) Representation of the partons' phase-space densities $F=\mathcal{N} \otimes \mathcal{P}$ as a convolution of the statistical density of dressed partons $\mathcal{N}$ with the spectral density $\mathcal{P}$ of each dressed parton, describing its intrinsic density of bare parton states as its quantum substructure.

variables, namely, its light-cone momentum $k_{j}^{+}=x_{j} P^{+}$with fraction $x_{j}$ of the total cell momentum, and its off-shellness (invariant virtuality) $k_{j}^{2}=k_{j}^{+} k_{j}^{-}-k_{j \perp}^{2} \equiv M^{2}\left(k_{j}\right)$. Its lightcone energy is $k_{j}^{-}=\left(k_{j}^{2}+k_{j \perp}^{2}\right) / k_{j}^{+} \simeq 0\left(k^{+2} \gg k^{2} \geq k_{\perp}^{2}\right)$, and one has, therefore,

$$
\begin{gathered}
k_{j}=\left(k_{j}^{+}, k_{j}^{2}\right)=\left(x_{j} P^{+}, k_{j}^{2}\right), \\
\frac{d^{4} k_{j}}{(2 \pi)^{4}}(2 \pi) \delta^{+}\left(k_{j}^{2}-M^{2}\left(k_{j}\right)\right)=\frac{1}{16 \pi^{2}} \frac{d x_{j}}{x_{j}} d k_{j}^{2} .
\end{gathered}
$$

The requirement Eq. (54) together with Eq. (50) hence translates to the parton level as $k_{j}^{+2} \gg k_{j}^{2} \geqslant \mu^{2}\left(r_{i}\right)$, for all partons $j$ within a given cell around $r_{i}$;

$$
Q^{2}\left(r_{i}\right) \geqslant k_{j}^{2} \geqslant \mu_{g q}^{2} \geqslant \mu^{2}\left(r_{i}\right) .
$$

Since $\lambda_{c}=\mu_{g q}^{-1}$ characterizes the maximum size of dressed partons, the ratio $\mu^{4}\left(r_{i}\right) / \mu_{g q}^{4}$ determines the minimum fraction of volume occupied by dressed partons in the cell. The quantum and kinetic space-time regions can now be defined as

$$
\Delta r_{\text {qua }}=\left[P^{+-1}, \mu^{-1}\right], \quad \Delta r_{\text {kin }}=\left[\mu^{-1}, \Lambda_{\mathrm{QCD}}^{-1}\right] .
$$

For large $P^{+}$and $Q^{2}$, quantum and kinetic length scales are well separated and the parton phase-space densities $F_{f}$ may be locally represented as a convolution of the kinetic, statistical density of dressed partons $\mathcal{N}_{f}$ of type $f=g, q$, with the quantum theoretical spectral density $\mathcal{P}_{f}$ of each dressed parton describing the intrinsic density of bare parton states as its quantum substructure, as depicted in Fig. 7(b):

$$
F_{f}\left(r_{i}, k\right)=F_{f}\left(r_{i}, k^{+}, k^{2}\right) \equiv \mathcal{N}_{f}\left(r_{i}, P^{+}, \mu_{g q}^{2}\right) \otimes \mathcal{P}_{f}\left(r_{i}, k^{+}, k^{2}\right),
$$

where the convolution of the statistical density $\mathcal{N}_{f}$ of dressed partons at the scale $\mu_{g q}^{2}$ with the spectral density $\mathcal{P}_{f}$, is defined as the average over the local space-time volume $\Omega\left(r_{i}\right)$ around $r_{i}$ of the densities,

$$
\begin{aligned}
\mathcal{N}_{f} \otimes \mathcal{P}_{f} \equiv & \frac{1}{\Omega\left(r_{i}\right)} \int_{\Omega\left(r_{i}\right)} d^{4} r^{\prime} \int \frac{d y}{y} \mathcal{N}_{f} \\
& \times\left(r^{\prime}, y P^{+}, \mu_{g q}^{2}\right) \mathcal{P}_{f}\left(r^{\prime}, \frac{x}{y}, k^{2}\right),
\end{aligned}
$$

with $\Omega\left(r_{i}\right) \simeq \mu\left(r_{i}\right)^{-4}, \quad P^{+}=P^{+}\left(r_{i}\right), \quad k^{+}=x P^{+}, \quad z=x / y$ $(0 \leqslant z \leqslant 1)$, and

$$
\mathcal{N}_{f}\left(r, y P^{+}, \mu_{g q}^{2}\right)=\frac{d N_{f}}{d^{4} r d \ln y} \delta\left(k^{2}-\mu_{g q}^{2}\right),
$$

$\mathcal{P}_{f}\left(r, z, k^{2}\right)=\sum_{f^{\prime}=g, q} \mathcal{P}_{f}^{f^{\prime}}=\sum_{f^{\prime}=g, q} \int_{\mu_{g q}^{2}}^{k^{2}} d k^{\prime 2} \frac{d n_{f}^{f^{\prime}}}{d \ln z d k^{\prime 2}}$.

This ansatz describes the multiparton system on the basis of treating each individual dressed parton as a composite particle of type $f$ with a substructure of a number of bare quanta $n_{f}^{f^{\prime}}$ of type $f^{\prime}$, weighted locally with the total number of dressed partons $N_{f}$ in a space-time cell. The spectral density $\mathcal{P}$ characterizes the intrinsic structure of a dressed parton state, whereas the quasiparticle density $\mathcal{N}$ describes the correlations and scatterings among those dressed partons. As will become clear later, the spectral densities $\mathcal{P}_{f}$ can indeed be identified with the QCD parton structure functions. The crucial quantities that control the cellular resolution in spacetime of the partons' substructure are the characteristic cell size $\mu^{-1}\left(r_{i}\right)$ and the minimum resolvable virtuality $\mu_{g q}^{2} \geqslant \mu^{2}$ of dressed partons in the cell, or alternatively the fractional space-time volume occupied, $\Delta \Omega / \Omega$ $=\mu^{4} /\left(N_{g q} \mu_{g q}\right)^{4}$, which determines how densely a cell may be populated without the partons overlapping. Hence the validity of the kinetic approximation, based on the separation of quantum and kinetic scales, is controlled by the choice of these quantities, which need not be constant but rather may be taken as space-time dependent, i.e., variable from cell to cell chosen such that the resolution is optimal. A convenient choice would be, for instance,

$$
\mu\left(r_{i}\right) \simeq \mu_{g q}
$$

which I will adopt in the following for lucidity, keeping in mind that $\mu_{g q}$ is not a free external parameter, but rather is to be understood as a dynamical, possibly space-timedependent quantity, which in principle should be determined self-consistently from screening effects. I will not address this latter issue here. 


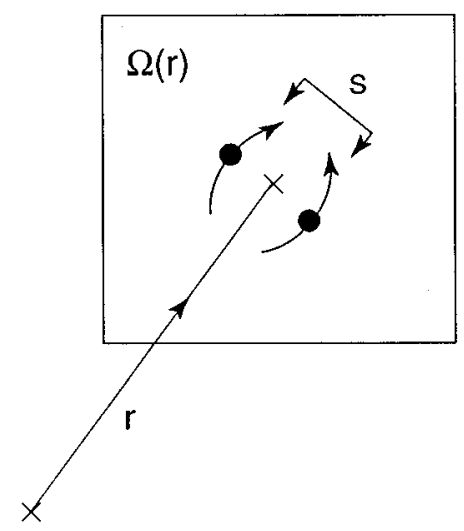

FIG. 8. In the cellular space-time picture, the "absolute" coordinate $r$ labels the kinetic space-time dependence $O\left(\Delta r_{\text {kin }}\right)$, whereas the "relative" coordinate $s$ measures the quantum spacetime distance $O\left(\Delta r_{\text {qua }}\right)$.

\section{B. Wigner transformation and the kinetic equations of motion}

Let me proceed, referring to Appendix B for details, by introducing center-of-mass and relative coordinates of two space-time points $x$ and $y$,

$$
r \equiv \frac{1}{2}(x+y), \quad s \equiv x-y,
$$

in terms of which one can express any two-point function $W(x, y) \equiv D_{\mu \nu}, S, \Pi_{\mu \nu}, \Sigma$, as

$$
W(x, y)=W\left(r+\frac{s}{2}, r-\frac{s}{2}\right)=W(r, s),
$$

and introduce its Wigner transform $W(r, k)$ as [13]

$$
\begin{gathered}
W(x, y)=\int \frac{d^{4} k}{(2 \pi)^{4}} e^{-i k \cdot s} W(r, k), \\
W(r, k)=\int d^{4} s e^{i k \cdot s} W(r, s),
\end{gathered}
$$

i.e., one Fourier transforms with respect to the relative coordinate $s$, the canonical conjugate to the momentum $k$. In the cell picture of space-time, the coordinate $r$ is the cell index that labels the kinetic space-time dependence $O\left(\Delta r_{\text {kin }}\right)$, whereas $s$ measures the quantum space-time distance $O\left(\Delta r_{\text {qua }}\right)$, as illustrated in Fig. 8. In homogeneous systems, such as the vacuum, translation invariance dictates that the dependence on $r$ drops out entirely, and the Wigner transforms then coincide with the momentum-space Fourier transforms of the Green functions and self-energies. In general, spatial inhomogeneities can be systematically accounted for by performing an expansion in terms of gradients $\partial_{r} \equiv \partial / \partial r^{\mu}$ :

$$
W(r+s, s) \simeq W(r, s)+s \cdot \partial_{r} W(r, s)+O\left[\left(s \cdot \partial_{r}\right)^{2}\right] .
$$

For quasihomogeneous or moderately inhomogeneous systems, such that $s \cdot \partial_{r} W \ll W$, the correlations between different cells will be small so that the propagators and selfenergies accordingly vary only slowly with $r$. One may then truncate the series (66) after the second term, and convert the quantum field equations of motion (44) and (45) into a set of kinetic equations by first performing the Wigner transformation (65) for all Green functions and self-energies, and then taking for Eqs. (44) and (45) the sum and difference of the two adjoint equations in their transformed representation.

This procedure (see Appendix B) yields two distinct equations for each of the Wigner transforms $D_{\mu \nu}$ and $S$ with rather different physical interpretations, which I will refer to as the renormalization equation and transport equation, respectively. The renormalization equations are obtained as

$$
\begin{aligned}
\left(k^{2}-\frac{1}{4} \partial_{r}^{2}\right) D_{a b}^{\mu \nu}(r, k)= & -d^{\mu \nu}(k) \delta_{a b} \hat{1}_{P} \\
& +\frac{1}{2}\left(\{\Pi, D\}_{+}\right)_{a b}^{\mu \nu}+\frac{i}{4} \mathcal{G}_{a b}^{\mu \nu(-)}, \\
\frac{1}{2}\left\{\gamma \cdot p, S_{i j}(r, p)\right\}_{+}= & \delta_{i j} \hat{1}_{P}-\frac{i}{2}\left(\left[\gamma \cdot \partial_{r}, S\right]_{-}\right)_{i j} \\
& +\frac{1}{2}\left(\{\Sigma, S\}_{+}\right)_{i j}+\frac{i}{4} \mathcal{F}_{i j}^{(-)},
\end{aligned}
$$

where $d^{\mu \nu}(k)$ is given by Eq. (8), $\partial_{r}^{2} \equiv \partial_{r} \cdot \partial_{r}$, and $[A, B]_{-} \equiv A B-B A, \quad\{A, B\}_{+} \equiv A B+B A$. The transport equations are found in the form

$$
\begin{aligned}
k \cdot \partial_{r} D_{a b}^{\mu \nu}(r, k)=- & \frac{i}{2}\left([\Pi, D]_{-}\right)_{a b}^{\mu \nu}+\frac{1}{4} \mathcal{G}_{a b}^{\mu \nu(+)}, \\
\frac{1}{2}\left\{\gamma \cdot \partial_{r}, S_{i j}(r, p)\right\}_{+}= & \frac{i}{2}\left([\gamma \cdot p, S]_{-}\right)_{i j} \\
& -\frac{i}{2}\left([\Sigma, S]_{-}\right)_{i j}+\frac{1}{4} \mathcal{F}_{i j}^{(+)} .
\end{aligned}
$$

In Eqs. (67) and (68) the self-energies $\Pi$ and $\Sigma$ are explicitly given by Eqs. (42) and (43), and the operator functions $\mathcal{G}$ and $\mathcal{F}$ on the right hand sides, which include the effects of spatial inhomogeneities to first order in the gradient expansion Eq. (66), $\operatorname{are}^{5}$

$$
\begin{gathered}
\mathcal{G}^{\mu \nu(-)}=\left[\partial_{k}^{\lambda} \Pi_{\sigma}^{\mu}, \partial_{\lambda}^{r} D^{\sigma \nu}\right]_{-}-\left[\partial_{r}^{\lambda} \Pi_{\sigma}^{\mu}, \partial_{\lambda}^{k} D^{\sigma \nu}\right]_{-}, \\
\mathcal{G}^{\mu \nu(+)}=\left\{\partial_{k}^{\lambda} \Pi_{\sigma}^{\mu}, \partial_{\lambda}^{r} D^{\sigma \nu}\right\}_{+}-\left\{\partial_{r}^{\lambda} \Pi_{\sigma}^{\mu}, \partial_{\lambda}^{k} D^{\sigma \nu}\right\}_{+}, \\
\mathcal{F}^{(-)}=\left[\partial_{p}^{\lambda} \Sigma, \partial_{\lambda}^{r} S\right]_{-}-\left[\partial_{r}^{\lambda} \Sigma, \partial_{\lambda}^{p} S\right]_{-}, \\
\mathcal{F}^{(+)}=\left\{\partial_{p}^{\lambda} \Sigma, \partial_{\lambda}^{r} S\right\}_{+}-\left\{\partial_{r}^{\lambda} \Sigma, \partial_{\lambda}^{p} S\right\}_{+} .
\end{gathered}
$$

For completeness, I note that the equations for quark Green functions can formally also be brought into a more familiar quadratic form, similar to the equations for the gluon Green

\footnotetext{
${ }^{5}$ Note that $\partial_{r}^{\mu} \equiv \partial / \partial k^{\mu}$ acts on a function $f(r, k)$ as the derivative with respect to the space-time coordinate, whereas $\partial_{k}^{\mu} \equiv \partial / \partial k^{\mu}$ and $\partial_{p}^{\mu} \equiv \partial / \partial p^{\mu}$ refer to the variation of four-momentum.
} 
functions, which exhibits the mass and drift term on the left hand side of the renormalization and transport equations, respectively:

$$
\begin{gathered}
\left(p^{2}-\frac{1}{4} \partial_{r}^{2}\right) S_{i j}(r, p)=(\gamma \cdot p+\Sigma) \delta_{i j} \hat{1}_{P}+\frac{1}{2}\left(\left\{\Sigma^{2}, S\right\}_{+}\right)_{i j} \\
+\frac{i}{4} \mathcal{A}_{i j}^{(+)}-\frac{1}{8} \mathcal{B}_{i j}^{(-)} \\
p \cdot \partial_{r} S_{i j}(r, p)=\frac{1}{2}\left(\gamma \cdot \partial_{r}\right) \delta_{i j} \hat{1}_{P}-\frac{i}{2}\left(\left[\Sigma^{2}, S\right]_{-}\right)_{i j} \\
+\frac{1}{4} \mathcal{A}_{i j}^{(-)}+\frac{i}{8} \mathcal{B}_{i j}^{(+)}
\end{gathered}
$$

where $\Sigma_{i j}=\delta_{i j} \Sigma$, and

$$
\begin{aligned}
\mathcal{A}^{( \pm)}= & \frac{1}{2}\left[(\gamma \cdot p+\Sigma)\left(\mathcal{F}^{(-)}+\mathcal{F}^{(+)}\right)\right. \\
& \left. \pm\left(\mathcal{F}^{(-)}-\mathcal{F}^{(+)}\right)(\gamma \cdot p+\Sigma)\right], \\
\mathcal{B}^{( \pm)}= & \frac{1}{2}\left[\left(\gamma \cdot \vec{\partial}_{r}\right)\left(\mathcal{F}^{(-)}+\mathcal{F}^{(+)}\right)\right. \\
& \left. \pm\left(\mathcal{F}^{(-)}-\mathcal{F}^{(+)}\right)\left(\gamma \cdot \overleftarrow{\partial}_{r}\right)\right] .
\end{aligned}
$$

As will be seen in the following, the renormalization equations (67) express the normalization conditions imposed by unitarity and renormalization group due to the quantum self-interactions, and redefine the bare quanta in terms of renormalized quasiparticles. The transport equations (68), on the other hand, describe the kinetic space-time evolution of the system of quasiparticles and their binary collisions.

The kinetic approximation of trading the Green functions $G(x, y)$ with their Wigner transforms $G(r, p)$ means in the picture of cellular space-time that inside a given cell carrying the space-time coordinate $r=(t, \vec{r})$ as a label, $G(r, p)$ equals the translation-invariant Fourier transform $G(p)$ of $G(x-y)$, but outside of the cell it is zero. In another cell $r^{\prime}$, the Wigner function $G\left(r^{\prime}, p^{\prime}\right)$ is determined by a different translation-invariant $G\left(p^{\prime}\right)$. Hence, when looking at the short-distance quantum fluctuations within a given spacetime cell around $r=\left(r^{0}, \vec{r}\right)$, one may approximate the spatial distribution of partons as being homogeneous and constant over the cell volume, and describe the short-range quantum dynamics in a translation-invariant manner. With the same accuracy of approximation, one can neglect in the quantum regime binary parton collisions, provided the mean free path $\lambda_{\mathrm{mf}}=\left(\sigma_{g q} F_{g q}\right)^{-1}$ in terms of the parton-parton cross sections $\sigma_{g q}$ and the local density $F_{g q}$ is large compared to the spatial spread of the quantum fluctuations, which is typically of the order of $1 / \sqrt{p^{2}}$. Hence the essential requirement $p^{2} \gg \lambda_{\mathrm{mf}}^{-2}$ can always be realized, if the particle energies are sufficiently large.

On the basis of these considerations, I first study the quantum theoretical aspects embodied in the renormalization equations (67) to obtain the renormalized gluon and quark propagators, and from this determine the momentum dependence of the phase-space densities $F_{g q}$, Eq. (58), associated with the variation of the parton structure functions. Subsequently, I will investigate the transport theoretical aspects of the statistical kinetic dynamics, described by transport equation (68), which determines the space-time variation of the phase-space densities $F_{g q}$ in terms of renormalized, dressed partons.

\section{The "physical representation" and strategy of solution}

Within the kinetic approximation, the goal is to obtain the best possible approximation to the complete propagators $G=D_{\mu \nu}, S$, starting from the case of noninteracting fields with the corresponding Wigner transformed Green functions $G_{(0)}=D_{(0)}^{\mu \nu}, S_{(0)}$. In this "free field" case, because of translational invariance, the $r$ dependence of the Green functions is homogeneous, and the equations of motion (67) and (68) reduce to

$$
\begin{gathered}
k^{2} D_{(0)}^{\mu \nu}(r, k)=-d^{\mu \nu}(k) \hat{1}_{P}, \quad k \cdot \partial_{r} D_{(0)}(r, k)=0, \\
p^{2} S_{(0)}(r, p)=\gamma \cdot p \hat{1}_{P}, \quad p \cdot \partial_{r} S_{(0)}=0,
\end{gathered}
$$

and the corresponding "free field" solutions of the four types of correlators $F,>,<, \bar{F}$ in Eqs. (27)-(31) are given by $[5,7]$

$$
\begin{aligned}
& D_{(0) \mu \nu}^{F}(r, k) \\
& =-d_{\mu \nu}(k)\left[\frac{1}{k^{2}+i \varepsilon}-2 \pi i F_{(0) g}(r, k) \delta\left(k^{2}-\mu_{g q}^{2}\right)\right], \\
& D_{(0) \mu \nu}^{>}(r, k)=-2 \pi i\left[-d_{\mu \nu}(k)\right]\left[\theta\left(+k_{0}\right)+F_{(0) g}(r, k)\right] \\
& \times \delta\left(k^{2}-\mu_{g q}^{2}\right), \\
& D_{(0) \mu \nu}^{<}(r, k)=-2 \pi i\left[-d_{\mu \nu}(k)\right]\left[\theta\left(-k_{0}\right)+F_{(0) g}(r, k)\right] \\
& \times \delta\left(k^{2}-\mu_{g q}^{2}\right), \\
& D_{(0) \mu \nu}^{\bar{F}}(r, k) \\
& =+d_{\mu \nu}(k)\left[\frac{1}{k^{2}-i \varepsilon}-2 \pi i F_{(0)}(r, k) \delta\left(k^{2}-\mu_{g q}^{2}\right)\right],
\end{aligned}
$$

where $d_{\mu \nu}(k)$ is defined by Eq. (8), and

$$
\begin{gathered}
S_{(0)}^{F}(r, p)=\frac{+1}{\gamma \cdot p+i \varepsilon}+2 \pi i F_{(0) q}(r, p) \delta\left(p^{2}-\mu_{g q}^{2}\right), \\
S_{(0)}^{>}(r, p)=+2 \pi i F_{(0) q}(r, p) \delta\left(p^{2}-\mu_{g q}^{2}\right), \\
S_{(0)}^{<}(r, p)=-2 \pi i\left[1-F_{(0) q}(r, p)\right] \delta\left(p^{2}-\mu_{g q}^{2}\right), \\
S_{(0)}^{\bar{F}}(r, p)=\frac{-1}{\gamma \cdot p-i \varepsilon}+2 \pi i F_{(0) q}(r, p) \delta\left(p^{2}-\mu_{g q}^{2}\right) .
\end{gathered}
$$

The scalar functions $F_{(0) g}$ and $F_{(0) q}$ are the free field analogues of Eq. (58) with the spectral densities $\mathcal{P}_{f}$ replaced by unity, 


$$
F_{(0) f}(r, k)=\mathcal{N}_{f}(r, k) \otimes 1=\left.\frac{d N_{f}}{d^{3} r d^{3} k}\right|_{r_{0}=t, k^{2}=\mu_{g q}^{2}}(f=g, q),
$$

i.e., the phase-space densities of gluons and quarks that measure the number of noninteracting quanta in a phase-space element $d^{3} r d^{4} k$ at a given time $t=r^{0}$ : It can be shown [8] that the function $F_{(0) g}\left(F_{(0) q}\right)$ is real, even, and the same for all four gluon (quark) correlators $D^{\mu} \nu(S)$ in Eqs. (74) and (75). For an ideal gas of gluons and quarks in equilibrium, for example, one finds $F_{(0)} \propto 1 /\left[\exp \left(\beta_{\mu} k^{\mu}\right) \pm 1\right]$ with $-(+)$ for gluons (quarks) and $\beta=\left(T^{-1}, \overrightarrow{0}\right)$ in the local rest frame. It is important to bear in mind that the presence of the functions $F_{g}$ and $F_{q}$ in Eqs. (74) and (75) even in the "free field" case is a direct consequence of the CTP formulation which incorporates initial state correlations due to a nontrivial density matrix $\hat{\rho}\left(t_{0}\right)$, Eq. (21), corresponding to $F\left(t_{0}, \vec{r}, p\right) \neq 0$, as opposed to the usual quantum field theory description, where $\hat{\rho}\left(t_{0}\right)=|0\rangle\langle 0|$ and $F\left(t_{0}, \vec{r}, p\right)$ vanishes. Evidently, in this latter case $G^{>}=G^{<}=0$ and $G^{F}=G^{\bar{F}^{\dagger}}$ at all times, so that the dynamics is described by the Feynman propagators $G^{F}$ alone.

More suitable for practical purposes, one may employ instead of the set $G^{F}, G^{>}, G^{<}, G^{\bar{F}}$, an equivalent set of the retarded (advanced) propagators $G^{R}\left(G^{A}\right)$ plus the correlation function $G^{C}$. The latter are directly connected with physical observable quantities, and are commonly referred to as the physical representation [7]. The functions $G^{R}, G^{A}$, and $G^{C}$ are obtained via the relations

$$
\begin{gathered}
G^{R}=G^{F}-G^{>}=G^{<}-G^{\bar{F}}, \quad G^{A}=G^{F}-G^{<}=G^{>}-G^{\bar{F}}, \\
G^{C}=G^{F}+G^{\bar{F}}=G^{<}+G^{>},
\end{gathered}
$$

which are most general identities that also hold in the presence of interactions. Because the fourth possible linear combination $G^{F}-G^{>}-G^{<}+G^{\bar{F}}$ is always identically zero, the three physical functions $G^{R}, G^{A}$, and $G^{C}$ form a complete alternative set that eliminates the overdetermination of the set $G^{F}, G^{>}, G^{<}, G^{\bar{F}}$. The "free field" forms of $G^{R}, G^{A}$, and $G^{C}$ corresponding to those of Eqs. (74) and (75) are

$$
\begin{gathered}
D_{(0) \mu \nu}^{R(A)}(r, k)=\frac{-d_{\mu \nu}(k)}{k^{2} \pm i \varepsilon k_{0}}, \quad S_{(0)}^{R(A)}(r, p)=\frac{1}{\gamma \cdot p \pm i \varepsilon p_{0}}, \\
D_{(0) \mu \nu}^{C}(r, k)=-2 \pi i\left[-d_{\mu \nu}(k)\right]\left[1+2 F_{(0) g}(r, k)\right] \\
\quad \times \delta\left(k^{2}-\mu_{g q}^{2}\right), \\
S_{(0)}^{C}(r, p)=-2 \pi i(\gamma \cdot p)\left[1-2 F_{(0) q}(r, p)\right] \delta\left(p^{2}-\mu_{g q}^{2}\right),
\end{gathered}
$$

where $+(-)$ in the denominators corresponds to the $R$ $(A)$. Generally speaking, the retarded and advanced functions characterize the quantum nature of parton states, whereas the correlation function describes the phase-space occupation of these states.

The preceding relations (77) are generally valid for any two-point function defined on the closed time path, and hence apply not only to the free field case, but also to the full Green functions $G=D_{\mu \nu}, S$, as well as to the self-energies $\mathcal{E}=\Pi_{\mu \nu}, \Sigma$. In matrix form, the correspondence between the representation (27) in terms of $G(\mathcal{E})$ and the physical rep- resentation denoted by $\breve{G}(\breve{\mathcal{E}})$ is given by a unitary transformation $\mathcal{U}, \quad$ a $2 \times 2$ matrix with $\mathcal{U}_{m n}=1 / \sqrt{2}$ for $m n=11,21,22$ and $\mathcal{U}_{12}=-1 / \sqrt{2}$ :

$$
\breve{G}=\mathcal{U} G \mathcal{U}^{-1}=\left(\begin{array}{cc}
0 & G^{A} \\
G^{R} & G^{C}
\end{array}\right), \quad \breve{\mathcal{E}}=\mathcal{U}^{-1} \mathcal{E} \mathcal{U}=\left(\begin{array}{cc}
\mathcal{E}^{C} & \mathcal{E}^{R} \\
\mathcal{E}^{A} & 0
\end{array}\right),
$$

where, in subtle contrast with Eq. (77),

$$
\begin{aligned}
& \mathcal{E}^{A}=\mathcal{E}^{F}+\mathcal{E}^{>}=-\mathcal{E}^{<}-\mathcal{E}^{\bar{F}}, \\
& \mathcal{E}^{R}=\mathcal{E}^{F}+\mathcal{E}^{<}=-\mathcal{E}^{>}-\mathcal{E}^{\bar{F}}, \\
& \mathcal{E}^{C}=\mathcal{E}^{F}+\mathcal{E}^{\bar{F}}=-\mathcal{E}^{<}-\mathcal{E}^{>} .
\end{aligned}
$$

The great advantage of this physical representation is that the dependence on the partons' phase-space densities $F_{g}$ and $F_{q}$ is essentially carried by the correlation functions $G^{C}$, whereas the dependence of the retarded and advanced functions $G^{R}$ and $G^{A}$ is weak. In the free field case, this separation of correlations is exact, as is evident from Eq. (78), such that the retarded and advanced functions do not depend at all on $F_{g}$ and $F_{q}$. In fact, even in the general case of interacting fields, this advantageous property becomes very suggestive when rewriting the renormalization and transport equations (67) and (68) in generic form for the individual Green function components:

$$
\begin{aligned}
\left\{G_{(0)}^{-1}, G^{R}-G^{A}\right\}_{+} & =-2\left(k^{2}-\frac{1}{4} \partial_{r}^{2}\right)\left(G^{R}-G^{A}\right) \\
= & \{\delta \mathcal{E}, \mathcal{P}\}_{+}+\{\Gamma, \delta G\}_{+}, \\
{\left[G_{(0)}^{-1}, G^{C}\right]_{-}=} & -2 i k \cdot \partial_{r} G^{C} \\
= & {\left[\mathcal{E}^{C}, \delta G\right]_{-}+\left[\delta \mathcal{E}, G^{C}\right]_{-} } \\
& +\frac{i}{2}\left(\left\{\mathcal{E}^{C}, \mathcal{P}\right\}_{+}+\left\{\Gamma, G^{C}\right\}_{+}\right),
\end{aligned}
$$

where

$$
\begin{array}{cc}
\delta G \equiv \operatorname{Re} G=\frac{1}{2}\left(G^{R}+G^{A}\right), & \delta \mathcal{E} \equiv \operatorname{Re} \mathcal{E}=\frac{1}{2}\left(\mathcal{E}^{R}+\mathcal{E}^{A}\right), \\
\mathcal{P} \equiv \operatorname{Im} G=i\left(G^{R}-G^{A}\right), & \Gamma \equiv \operatorname{Im} \mathcal{E}=i\left(\mathcal{E}^{R}-\mathcal{E}^{A}\right)
\end{array}
$$

are the real and imaginary components of the retarded and advanced Green functions and self-energies, whereas

$$
G^{C}=G^{<}+G^{>}, \quad \mathcal{E}^{C}=-\left(\mathcal{E}^{<}+\mathcal{E}^{>}\right)
$$

are the real correlation functions and corresponding selfenergies. The physical significance of Eqs. (81) and (82) is the following. Equation (81) determines the state of a dressed parton with respect to its virtual fluctuations and real emission (absorption) processes, corresponding to the real and imaginary parts of the retarded and advanced selfenergies. Equation (82), on the other hand, characterizes the correlations among different dressed parton states, and the self-energies appear here in two distinct ways. The first two terms on the right hand side account for scatterings between quasiparticle states, i.e., dressed partons, whereas the last two terms incorporate the renormalization effects which result from the fact that the dressed partons between collisions do not behave as free particles, but change their dynamical structure due to virtual fluctuations, as well as real emission 
and absorption of quanta. For this reason $\mathcal{E}^{R(A)}$ are called radiative self-energies, and $\mathcal{E}^{C}$ is termed the collisional selfenergy. As shown by Kadanoff and Baym [5], the imaginary parts of the retarded and advanced Green functions and selfenergies (83) are just the spectral density $\mathcal{P}$, giving the probability for finding an intermediate multiparticle state in the dressed parton, and, respectively, the decay width $\Gamma$, describing the dissipation of the dressed parton. The solution of Eqs. (81) and (82) can be obtained in complete analogy to the detailed derivation given by Kadanoff and Baym [5]. One finds that the solution for the spectral density $\mathcal{P}$ has the same formal structure as in equilibrium, namely,

$$
\mathcal{P}(r, k)=\frac{\Gamma}{k^{2}-\delta \mathcal{E}+(\Gamma / 2)^{2}} \equiv \Delta \mathcal{P}_{\delta \mathcal{E}}+\Delta \mathcal{P}_{\Gamma}
$$

describing the particle density in terms of the finite width $\Gamma$ and the dynamical mass term $\delta \mathcal{E}$ (which in the "free field" case are $\Gamma=\delta \mathcal{E}=0$, corresponding to an on-shell, classically stable particle). On the right hand side of Eq. (85), the second form exhibits the physical meaning more suggestively in terms of the "wave function" renormalization $\left(\Delta \mathcal{P}_{\delta \mathcal{E}}\right)$ due to virtual fluctuations, and the dissipative parts $\left(\Delta \mathcal{P}_{\Gamma}\right)$ due to real emission (absorption) processes. This separation will prove convenient later. ${ }^{6}$ The spectral density $\mathcal{P}$ satisfies the sum rule $[7,5]$

$$
\begin{aligned}
1 & =\frac{1}{P^{+}} \int \frac{d k^{+}}{2 \pi k^{+}} T^{++} \mathcal{P}\left(r, k^{+}, k^{2}\right) \\
& =\frac{1}{P^{+}} \int \frac{d k^{+}}{2 \pi} k^{+} \mathcal{P}\left(r, k^{+}, k^{2}\right),
\end{aligned}
$$

which is an implicit consequence of unitarity, and requires that the total light-cone momentum of the spectral density of the internal bare partons must be equal to the dressed partons' momentum. For example, in the "free field" case, i.e., in the absence of interactions, one has $\Delta \mathcal{P}_{\delta \mathcal{E}}$ $=\delta\left(1-k^{+} / P^{+}\right)$and $\Delta \mathcal{P}_{\Gamma}=0$ with $k^{2}=\mu_{g q}^{2}$, so that $\mathcal{P} \rightarrow \mathcal{P}_{(0)}$ describes a single "on-shell" parton state

$$
\mathcal{P}_{(0)}\left(r, k^{+}, k^{2}\right)=\delta\left(k^{2}-\mu_{g q}^{2}\right) \delta\left(1-\frac{k^{+}}{P^{+}}\right),
$$

on the "mass shell" $k^{2}=\mu_{g q}^{2}$, and carrying the total lightcone energy $k^{+}=P^{+}{ }^{7}$ This is nothing but the fact that the presence of a pole in the Green function means the presence of a particle, stable if it occurs for real $k^{2}$, unstable if it occurs for complex $k^{2}$, as in the Breit-Wigner formula (85). The generalization of Eq. (87) to the case of interactions, in which, as advocated before, a dressed parton may be visualized as a substructured particle with a fluctuating number of bare quanta intermediately present in its wave function, is straightforward. A dressed parton has now a "blurred" mass shell, because its internal excitations fluctuate due to virtual

\footnotetext{
${ }^{6}$ The formula $(85)$ holds for both spacelike $\left(k^{2}<0\right)$ and timelike $\left(k^{2}>0\right)$ momenta. If $k^{2}$ is spacelike then the imaginary part $\Delta \mathcal{P}_{\Gamma}$ vanishes, so that $\mathcal{P}$ is purely real. On the other hand, if $k^{2}$ is timelike then both $\Delta \mathcal{P}_{\delta \mathcal{E}}$ and $\Delta \mathcal{P}_{\Gamma}$ contribute, and so $\mathcal{P}$ is complex.

${ }^{7}$ Note that for the choice Eq. (62), the fraction $z=x / y$ in the defining equation for $\mathcal{P}$, Eq. (61), reduces to $z=x$.
}

and real emission (absorption) processes of its bare daughter partons. The spectrum of these quantum excitations will have a finite extension around $\mu_{g q}^{2}$, described by the real part $\delta \mathcal{E}$ of the self-energy, with a width $\Gamma$, described by the imaginary part and being inversely proportional to the lifetime of the particular parton state. Hence, one may write formally, instead of Eq. (87),

$$
\mathcal{P}(r, k)=\delta\left(k^{2}-\mathcal{M}^{2}\left(r, k^{+}, k^{2}\right)\right), \quad \mathcal{M}^{2}=\delta \mathcal{E}-\frac{\Gamma^{2}}{4},
$$

where $\delta \mathcal{E}$ and $\Gamma$ are given in terms of the real and imaginary parts of the retarded and advanced self-energies [Eq. (83)]. This representation serves to maintain the analogy with the free field case, for which one has an immediate intuition. However, instead of a simple mass-shell condition, the argument of the $\delta$ function now expresses a nontrivial functional dependence of the spectrum on $k^{+}$and $k^{2}$ and, in general, on space-time $r$. The solution of this implicit equation determines the spectral density $\mathcal{P}$, which is the subject of Sec. III D.

Once the spectral density is known, the correlation function $G^{C}$ is given by the generic expression [5]

$$
\begin{aligned}
G^{C}(r, k) & =-2 \pi i[1 \pm 2 \mathcal{N}(r, k)] \otimes \mathcal{P}(r, k) \\
& =-2 \pi i[1 \pm 2 F(r, k)] \delta\left(k^{2}-\mathcal{M}^{2}(r, k)\right),
\end{aligned}
$$

where $+(-)$ is for gluons (quarks). It reduces to the free field form when $\mathcal{P}$ is replaced by $\mathcal{P}_{(0)}$, Eq. (87), so that $G_{(0)}^{C}=-2 \pi i[1 \pm 2 \mathcal{N}] \delta\left(k^{2}-\mu_{g q}^{2}\right)$ $=-2 \pi i\left[1 \pm 2 F_{(0)}\right] f \times \delta\left(k^{2}-\mu_{g q}^{2}\right)$ becomes an on-shell distribution, as in Eqs. (78). The theoretical basis for the previous, more physically motivated, ansatz [Eq. (58)] for the parton phase-space distributions $F$ becomes evident now: It is the logical generalization of the free field forms [Eq. (78)] to include renormalization effects and dissipation in terms of nontrivial spectral densities, or parton structure functions, which embody the underlying quantum dynamics. In this sense the Wigner functions $F$ are the quantum kinetic extension of the classical particle phase-space distributions.

Following this strategy, I will now proceed on the basis of the factorized ansatz [Eq. (58)] for the gluon and quark densities $F_{f}$ in terms of the quasiparticle distributions $\mathcal{N}_{f}$ with the spectral densities $\mathcal{P}_{f}$, i.e., the presumption that the separation between quantum and kinetic scales allows a distinct treatment of the intrinsic quantum fluctuations of dressed partons and the kinetic correlations among them. In contrast to Eq. (78), the poles of the retarded and advanced Green functions are shifted by the real and imaginary parts of the self-energies $\mathcal{E}^{R(A)}$, and in the expression for the correlation functions the $\delta$ function is replaced by the spectral density $\mathcal{P}$. Introducing the scalar functions for $\hat{\Pi}$ for the gluon and $\hat{\Sigma}$ for the quark self-energies through

$$
\Pi_{a b}^{\mu \nu}=\delta_{a b}\left(k^{\mu} k^{\nu}-g^{\mu \nu} k^{2}\right) \hat{\Pi}, \quad \Sigma_{i j}=\delta_{i j} p^{2} \hat{\Sigma},
$$

instead of Eq. (78) one has now 


$$
\begin{gathered}
D_{\mu \nu}^{R(A)}(r, k)=\frac{-d_{\mu \nu}(k)}{k^{2}\left(1-\hat{\Pi}^{R(A)}\right)}, \\
S^{R(A)}(r, p)=\frac{\gamma \cdot p}{p^{2}\left(1-\gamma \cdot p \hat{\Sigma}^{R(A)}\right)}, \\
D_{\mu \nu}^{C}(r, k)=-2 \pi i\left[-d_{\mu \nu}(k)\right]\left[1+2 \mathcal{N}_{g}(r, k)\right] \otimes \mathcal{P}_{g}(r, k) \\
=-2 \pi i\left[-d_{\mu \nu}(k)\right]\left[1+2 F_{g}(r, k)\right] \\
\times \\
\delta\left(k^{2}-\mathcal{M}_{g}^{2}(r, k)\right), \\
S^{C}(r, p)=-2 \pi i(\gamma \cdot p)\left[1-2 \mathcal{N}_{q}(r, p)\right] \otimes \mathcal{P}_{q}(r, p) \\
=-2 \pi i(\gamma \cdot p)\left[1-2 F_{q}(r, p)\right] \delta\left(p^{2}-\mathcal{M}_{q}^{2}(r, p)\right) .
\end{gathered}
$$

The two-step strategy that I will follow in the next sections is the following.

(1) In Sec. III D, the renormalization equations (67) will be solved for the retarded (advanced) Green functions

$$
\begin{gathered}
\left(D_{R(A)}^{-1}\right)_{a b}^{\mu \nu}(r, k)=\left(D_{(0) R(A)}^{-1}\right)_{a b}^{\mu \nu}-\left(\Pi_{R(A)}\right)_{a b}^{\mu \nu}, \\
\left(S_{R(A)}^{-1}\right)_{i j}(r, p)=\left(S_{(0) R(A)}^{-1}\right)_{i j}-\left(\Sigma_{R(A)}\right)_{i j},
\end{gathered}
$$

which determine the spectral densities $\mathcal{P}_{g}$ and $\mathcal{P}_{q}$ in terms of the radiative self-energies $\Pi_{\mu \nu}^{R(A)}$ and $\Sigma^{R(A)}$.

(2) In Sec. III E the transport equations will be solved for the correlation functions

$$
\begin{gathered}
D_{C a b}^{\mu \nu}(r, k)=-D_{R a a^{\prime}}^{\mu \mu^{\prime}}\left[\left(D_{(0) C}^{-1}\right)_{a^{\prime} b^{\prime}}^{\mu^{\prime} \nu^{\prime}}-\left(\Pi_{C}\right)_{a^{\prime} b^{\prime}}^{\mu^{\prime} \nu^{\prime}}\right] D_{A b^{\prime} b}^{\nu^{\prime} \nu^{\prime}}, \\
S_{C i j}(r, p)=-S_{R i i^{\prime}}\left[\left(S_{(0) C}^{-1}\right)_{i^{\prime} j^{\prime}}-\left(\Sigma_{C}\right)_{i^{\prime} j^{\prime}}\right] S_{A i^{\prime} j},
\end{gathered}
$$

which determine the parton phase-space distributions $F_{g}$ and $F_{q}$ by the collisional self-energies $\Pi_{\mu \nu}^{C}$ and $\Sigma^{C}$, in conjunction with the spectral densities $\mathcal{P}_{g}$ and $\mathcal{P}_{q}$.

\section{Quantum dynamics and renormalization equations}

As advocated above, when addressing renormalization effects and dissipative quantum dynamics, it is appropriate to focus on the retarded and advanced propagators and the imaginary parts of the self-energies, which embody the short-distance propagation of quantum fluctuations. Furthermore, on quantum scales, one can neglect the $r$ dependence, and thus ignore in this regime the functions $\mathcal{G}$ and $\mathcal{F}$ in Eqs. (67) and (68). Then, by performing the transformation of the Wigner transformed Green functions $D_{\mu \nu}, S$ and selfenergies $\Pi_{\mu \nu}, \Sigma$ to the physical representation via Eq. (79), one obtains simplified equations for the retarded (advanced) functions (cf. Appendix B). The renormalization equations reduce to the form

$$
\begin{gathered}
\left(k^{2}-\frac{1}{4} \partial_{r}^{2}\right) D_{a b}^{\mu \nu R(A)}(r, k) \\
=-d^{\mu \nu}(k) \delta_{a b}+\frac{1}{2}\left(\Pi^{R(A)} D^{R(A)}+D^{R(A)} \Pi^{R(A)}\right)_{a b}^{\mu \nu}, \\
\frac{1}{2}\left\{\gamma \cdot p, S_{i j}^{R(A)}(r, p)\right\}_{+}=\delta_{i j}+\frac{1}{2}\left(\Sigma^{R(A)} S^{R(A)}+S^{R(A)} \Sigma^{R(A)}\right)_{i j} .
\end{gathered}
$$

To solve the equations (95), it is suggestive in view of Eq. (78) to parametrize the renormalized, "dressed" propagators $D_{\mu \nu}^{R}$ and $S^{R}$ on account of their Lorentz structure as $[27,28]$

$$
\begin{aligned}
& D_{a b}^{\mu \nu R(A)}(r, k)= \delta_{a b} \frac{-d^{\mu \nu}(k)}{k^{2}\left[1-\hat{\Pi}^{R(A)}(r, k)\right]} \\
& \equiv \Delta_{g}\left(r, k^{2}, \kappa_{k}\right) \delta_{a b} \frac{-d_{\mu \nu}(k)}{k^{2} \pm i \varepsilon k_{0}}+\cdots, \gamma \cdot p \\
& S_{i j}^{R(A)}(r, p)= \delta_{i j} \frac{\gamma p^{2}\left[1-\gamma \cdot p \hat{\Sigma}^{R(A)}(r, p)\right]}{\equiv} \\
& \equiv \Delta_{q}\left(r, p^{2}, \kappa_{p}\right) \delta_{i j} \frac{\gamma \cdot p}{p^{2} \pm i \varepsilon p_{0}} \\
&+\widetilde{\Delta}_{q}\left(r, p^{2}, \kappa_{p}\right) \delta_{i j} \frac{\gamma \cdot n}{n \cdot p}+\cdots,
\end{aligned}
$$

where $d_{\mu \nu}(k)=g_{\mu \nu}-\left(n_{\mu} k_{\nu}+n_{\nu} k_{\mu}\right) /(n \cdot k)$ as before, the scalar self-energy functions $\hat{\Pi}^{R}$ and $\hat{\Sigma}^{R}$ are defined by Eq. (90), and $\kappa$ implicitly accounts for the dependence of $\Delta_{g}$ and $\Delta_{q}$ on the coordinate $r$, which is conjugate to $k^{+}$. The function $\kappa$ is of the order of the large light-cone momentum $k^{+}$ squared (cf. Appendix C):

$$
\kappa_{k}^{2} \equiv \frac{(n \cdot k)^{2}}{n^{2}} \simeq k^{+2}, \quad n^{2} \ll 1 .
$$

The renormalization functions $\Delta_{g}\left(\Delta_{q}\right)$ account for the modifications of the "bare" propagators (78) due to the selfinteractions embodied in $\Pi(\Sigma)$. The third function $\widetilde{\Delta}_{q}$ turns out to be proportional to $\Delta_{q}$ (cf. Appendix C). They are normalized in accord with the condition (56), such that

$$
\left.\Delta_{g}\left(r, k^{2}, \kappa_{p}\right)\right|_{k^{2}=\mu_{g q}^{2}}=\left.\Delta_{q}\left(r, p^{2}, \kappa_{p}\right)\right|_{p^{2}=\mu_{g q}^{2}}=1,
$$

meaning that a gluon or quark is considered as a maximally dressed particle [in the sense of the applicability of Eq. (56)], corresponding to the invariant scale $\mu_{g q}$, which may be called the dressed partons' mass shell.

It is well known [27] that other contributions to the propagators in Eq. (96), indicated by the ellipses, are strongly suppressed $^{8}$ in light-cone-dominated processes $Q^{2} \rightarrow \infty$ by inverse powers of $Q^{2}$. In fact, this feature is the very foundation of the QCD parton description within the (modified) leading log approximation (MLLA) [27,28], where the renormalization problem reduces to a multiplication of the bare propagators and vertices by scalar functions. That is, as de-

\footnotetext{
${ }^{8}$ Note the benefit of the employed gauge $n_{\mu} A^{\mu}=0$ for the gluon field, Eq. (3): by suitable choice of the vector $n^{\mu}$ such that $n^{2} \ll 1$, one can concentrate the short-distance quantum fluctuations to arbitrary proximity around the light cone, $n^{2} \rightarrow 0$, i.e., $\kappa_{k} \rightarrow \infty$, corresponding to the asymptotic limit $k^{+} \rightarrow \infty$. In this regime the leading $\log$ singularities of the propagators give the dominant contributions and the ellipses in Eqs. (96) and (100) can be neglected because they do not generate leading logs.
} 
picted in Fig. 9, with respect to the Wigner transforms of the self-energies Eqs. (42) and (43),

$$
\begin{aligned}
\Pi_{a b}^{\mu \nu R(A)}(r, k)= & -\frac{g_{s}^{2}}{2} \int \frac{d^{4} k^{\prime}}{(2 \pi)^{4} i} f_{a a^{\prime} c^{\prime}} \lambda_{\mu \mu^{\prime} \sigma}\left(-k,-k+k^{\prime}, k^{\prime}\right) \\
& \times D_{c d}^{\sigma \tau R(A)}\left(r, k^{\prime}\right) \Lambda_{d b^{\prime} b}^{\tau \nu^{\prime} \nu}\left(-k^{\prime}, k-k^{\prime}, k\right) \\
& \times D_{a^{\prime} b^{\prime}}^{\mu^{\prime} \nu^{\prime} R(A)}\left(r, k-k^{\prime}\right) \\
& -g_{s}^{2} N_{f} \int \frac{d^{4} k^{\prime}}{(2 \pi)^{4} i} \gamma_{\mu} T_{l n}^{a} S_{n n^{\prime}}^{R(A)}\left(r,-k^{\prime}+k\right) \\
& \times \Xi_{n^{\prime} l^{\prime} \nu}^{b}\left(-k^{\prime}+k, k, k^{\prime}\right) S_{l l^{\prime}}^{R(A)}\left(r, k^{\prime}\right),
\end{aligned}
$$

$$
\Sigma_{i j}^{R(A)}(r, p)=g_{s}^{2} \int \frac{d^{4} k^{\prime}}{(2 \pi)^{4} i} \gamma_{\sigma} T_{i i^{\prime}}^{c} S_{i^{\prime} j^{\prime}}^{R(A)}\left(r, p-k^{\prime}\right)
$$

$$
\times \Xi_{j^{\prime} j \tau}^{d}\left(p-k^{\prime}, p, k^{\prime}\right) D_{c d}^{\sigma \tau R(A)}\left(r, k^{\prime}\right),
$$

one can represent the $q q g$ and $g g g$ vertex functions $\Gamma$ $(\Lambda)$, Eq. (37), as multiplicative renormalization functions:

$$
\begin{aligned}
\Gamma_{i j}^{\mu a}\left(r ; p_{1}, p_{2}, k_{3}\right)= & \gamma^{\mu} T_{i j}^{a} V_{q q g}\left(r ; p_{1}^{2}, p_{2}^{2}, k_{3}^{2}, \kappa_{1}, \kappa_{2}, \kappa_{3}\right) \\
& +\cdots, \\
\Lambda_{\mu \nu \lambda}^{a b c}\left(r ; k_{1}, k_{2}, k_{3}\right)= & f^{a b c} g_{\mu \lambda} k_{\nu} V_{g g g}\left(r ; k_{1}^{2}, k_{2}^{2}, k_{3}^{2}, \kappa_{1}, \kappa_{2}, \kappa_{3}\right) \\
& +\cdots,
\end{aligned}
$$

where $k_{\nu}$ is understood as the momentum associated with the intermediate of the three gluon virtualities, i.e., $k_{\nu}=k_{2 \nu}$ if $k_{1}^{2}<k_{2}^{2}<k_{3}^{2}$, etc. Analogous to Eq. (98), the normalization conditions are

$\left.V_{q q g}\left(r ; p_{i}^{2}, \kappa_{i}\right)\right|_{p_{i}^{2}=\mu_{g q}^{2}}=\left.V_{g g g}\left(r ; k_{i}^{2}, \kappa_{i}\right)\right|_{k_{i}^{2}=\mu_{g q}^{2}}=1$.

Employing these definitions in the renormalization equations (95) and the expressions for self-energies given by Eqs. (42) and (43), and differentiating the inverse of the propagators $D_{\mu \nu}^{R(A)}$ and $S^{R(A)}$ with respect to the gluon and quark virtuality, respectively,

$$
\begin{gathered}
\frac{\partial}{\partial k^{2}}\left\{-d^{\mu \nu}(k)\left[D^{R(A)}(r, k)\right]_{\mu \nu}^{-1}\right\}=1-\frac{\partial}{\partial k^{2}} \hat{\Pi}^{R(A)}(r, k), \\
\frac{\partial}{\partial p^{2}}\left\{\gamma \cdot p\left[S^{R(A)}(r, p)\right]^{-1}\right\}=1-\frac{\partial}{\partial p^{2}} \hat{\Sigma}^{R(A)}(r, p),
\end{gathered}
$$

one obtains the following determining equations for the momentum dependence of the scalar self-energy functions [Eq. (90)] $\hat{\Pi}^{R(A)}$ and $\hat{\Sigma}^{R(A)}$ to order $g_{s}^{2}$ in terms of the renormalization functions $\Delta_{g}$ and $\Delta_{q}$ (for more details, see Appendix C):

$$
\begin{aligned}
k^{2} \frac{\partial}{\partial k^{2}} \hat{\Pi}^{R(A)}(r, k)= & \frac{g_{s}^{2}}{2} C_{A}(2 \pi i) \int \frac{d^{4} k^{\prime}}{(2 \pi)^{4} i} \int_{r_{0}-1 /[2 \mu(r)]}^{r_{0}+1 /(2 \mu)} d \tau \mathcal{T}\left(k^{\prime} \tau\right) \frac{\partial \Delta_{g}\left(r, k^{\prime}\right)}{\partial k^{\prime 2}} \frac{\partial}{\partial k^{\prime \prime 2}} \\
& \times\left[V_{g g g}^{2}\left(r ; k^{2}, k^{\prime 2}, k^{\prime \prime 2}, \kappa_{k}, \kappa_{k^{\prime}}, \kappa_{k^{\prime \prime}}\right) \Delta_{g}\left(r, k^{\prime \prime}\right)\right] \mathcal{U}_{g}^{g g}\left(k^{\prime}, k^{\prime \prime}, n\right) \\
& -g_{s}^{2} \operatorname{Tr} N_{f}(2 \pi i) \int \frac{d^{4} p^{\prime}}{(2 \pi)^{4} i} \int_{r_{0}-1 /(2 \mu)}^{r_{0}+1 /(2 \mu)} d \tau \mathcal{T}\left(p^{\prime} \tau\right) \frac{\partial \Delta_{q}\left(r, p^{\prime}\right)}{\partial p^{\prime 2}} \frac{\partial}{\partial p^{\prime \prime}} \\
& \times\left[V_{g q q}^{2}\left(r ; k^{2}, p^{\prime 2}, p^{\prime \prime 2}, \kappa_{k}, \kappa_{p^{\prime}}, \kappa_{p^{\prime \prime}}\right) \Delta_{q}\left(r, p^{\prime \prime}\right)\right] \mathcal{U}_{g}^{q q}\left(p^{\prime}, p^{\prime \prime}, n\right), \\
p^{2} \frac{\partial}{\partial p^{2}} \hat{\Sigma}^{R(A)}(r, p)= & -g_{s}^{2} C_{F}(2 \pi i) \int \frac{d^{4} p^{\prime}}{(2 \pi)^{4} i} \int_{r_{0}-1 /(2 \mu)}^{r_{0}+1 /(2 \mu)} d \tau \mathcal{T}\left(p^{\prime} \tau\right) \frac{\partial \Delta_{q}\left(r, p^{\prime}\right)}{\partial p^{\prime 2}} \frac{\partial}{\partial k^{\prime \prime 2}} \\
& \times\left[V_{q q g}^{2}\left(r ; p^{2}, p^{\prime 2}, k^{\prime \prime 2}, \kappa_{p}, \kappa_{p^{\prime}}, \kappa_{k^{\prime \prime}}\right) \Delta_{g}\left(r, k^{\prime \prime}\right)\right] \mathcal{U}_{q}^{q g}\left(p^{\prime}, k^{\prime \prime}, n\right),
\end{aligned}
$$

plus terms $O\left(n^{2}\right)$ which can be neglected for $n^{2} \ll 1$. The constants $\quad C_{A} \delta_{a b}=f_{a c d} f_{b c d}=N_{c} \delta_{a b}, \quad T_{R} \delta_{a b}=\operatorname{Tr}\left(T^{a} \cdot T^{b}\right)$ $=\frac{1}{2} \delta_{a b}$, and $C_{F} \delta_{i j}=\left(T^{a} \cdot T^{a}\right)_{i j}=\left[\left(N_{c}^{2}-1\right) 2 N_{c}\right] \delta_{i j}$ arise from summing over color indices, and $N_{f}$ is the number of quark flavors. As explained in Appendix $\mathrm{C}$, the time integral $\int d \tau$ on the right hand sides extends over the finite time slice $\mu^{-1} \equiv \mu^{-1}(r)$ of the space-time cell around $r$, weighted by the function $\mathcal{T}$ which satisfies

$$
\int_{0}^{\infty} d \tau \mathcal{T}\left(p^{\prime} \tau\right)=1
$$

e.g., $\mathcal{T}=p^{\prime} \exp \left(-p^{\prime} \tau\right)$. With reference to Ref. [37], where an elaborate presentation of the time dependence of parton densities in the framework of QCD evolution is given, it suffices here to note that the effect of the finite time slice integration $\int d \tau$ is to impose the compatibility condition on space-time 
(a)

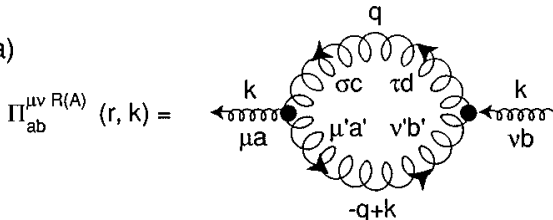

q

(b)

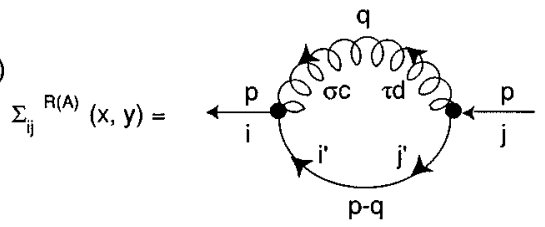

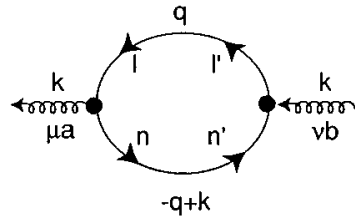

$-q+k$
FIG. 9. The "radiative" self-energies in oneloop approximation, Eqs. (99): (a) the retarded (advanced) gluon self-energies $\Pi_{\mu \nu}^{R(A)}$, and (b) the retarded (advanced) quark self-energies $\Sigma^{R(A)}$. and energy-momentum variables as constrained by the uncertainty principle: it limits the range of virtualities $k^{\prime 2}$ such that within the finite time interval $\Delta r_{0}=\mu^{-1}$ only those fluctuations $k \rightarrow k^{\prime} k^{\prime \prime}$ are resolvable that are sufficiently short living, with proper lifetime $\tau_{0} \simeq 1 / k^{\prime}$ and $\gamma \tau_{0}$ $\simeq k^{+} / k^{\prime} 2<\Delta r_{0}$. Finally, the functions $\mathcal{U}_{f}^{f^{\prime} f^{\prime \prime}}\left(k^{\prime}, k^{\prime \prime}, n\right)$ represent the squared matrix elements for the virtual decay processes $k \rightarrow k^{\prime} k^{\prime \prime}$. Their explicit form is given in Appendix C.

In the cellular space-time picture, the momenta of partons in a given cell around $r$ are per design limited by the condition (56), such that

$$
P^{+2}(r) \geqslant k^{+2} \geqslant k^{2} \geqslant \mu_{g q}^{2} \geqslant \mu^{2}(r) .
$$

As explained in Appendix $\mathrm{C}$, by employing this condition and introducing the fractional light-cone momenta of the daughter partons in the process $k \rightarrow k^{\prime} k^{\prime \prime}$,

$$
\begin{gathered}
z_{k^{\prime}} \equiv z=\frac{k^{\prime+}}{k^{+}}, \quad z_{k^{\prime \prime}} \equiv 1-z=\frac{k^{\prime+}}{k^{+}}, \\
d^{4} k^{\prime}=\frac{\pi}{2} d k^{\prime 2} d k^{\prime \prime} 2 d z \quad \theta\left(k^{2}-\frac{k^{\prime 2}}{z}-\frac{k^{\prime \prime 2}}{1-z}\right),
\end{gathered}
$$

the integrals (103) and (104) are readily evaluated to leading $\log$ accuracy. Solving for the renormalization functions $\Delta_{g}$ and $\Delta_{q}$, and taking $\kappa_{k}=k^{+2}$ and $\kappa_{p}=p^{+2}$ from Eq. (97), the result is (cf. Appendix C)

$$
\begin{aligned}
\Delta_{g}\left(r, k^{2}, k^{+2}\right)= & \exp \left\{-\int_{k^{2}}^{k^{+2}} \frac{d k^{\prime 2}}{k^{\prime 2}} \int_{0}^{1} d z A\left(r, z, k^{\prime 2}\right)\right. \\
& \left.\times\left(\frac{1}{2} \gamma_{g}^{g g}(z, \epsilon)+\gamma_{g}^{q q}(z, \epsilon)\right)\right\}, \\
\Delta_{q}\left(r, p^{2}, p^{+2}\right) & \exp \left\{-\int_{p^{2}}^{p^{+2}} \frac{d p^{\prime 2}}{p^{\prime 2}} \int_{0}^{1} d z A\left(r, z, p^{\prime 2}\right) \gamma_{q}^{q g}(z, \epsilon)\right\},
\end{aligned}
$$

where

$$
A\left(r, k^{2}, z\right) \simeq \frac{\alpha_{s}\left[(1-z) k^{2}\right]}{2 \pi} \theta\left(\frac{z k^{2}}{k^{+}}-\mu(r)\right)
$$

is the effective local coupling strength ${ }^{9}$ averaged over a cell centered around $r$ of space-time extent $\Omega(r) \simeq \Delta^{4} r$, and $\left.\alpha_{s}\left(q^{2}\right)=b \ln \left(q^{2} / \Lambda_{\mathrm{QCD}}^{2}\right)\right]^{-1}, \quad b=11 N_{c}-2 N_{f}$. The function $A\left(r, z, k^{2}\right)$ reflects the fact that in cellular space-time the relevant quantum fluctuations are restricted by the uncertainty principle, as embodied in the $\theta$-function term: $k^{2} \leqslant \mu^{2}=1 /(\Delta r)^{2}, k^{0} \leqslant 1 / \Delta r^{0}$, with $\Delta r^{0}=k^{+} / k^{2}$.

The functions $\gamma(z, \epsilon)$ in Eq. (109) involve, at leading-log level, the standard Dokshitzer-Gribov-Lipatov-AltarelliParisi (DGLAP) kernels [27], carrying an explicit $k^{+}$dependence arising from the dependence on $\kappa \simeq k^{+2}$,

$$
\begin{aligned}
\gamma_{g}^{g g}(z, \epsilon)= & 2 C_{A}\left(\frac{z}{1-z+\epsilon\left(\kappa_{k}\right)}+\frac{1-z}{z}+z(1-z)\right) \\
& \gamma_{g}^{q q}(z, \epsilon)=\frac{1}{2}\left[z^{2}+(1-z)^{2}\right] \\
& \gamma_{q}^{q g}(z, \epsilon)=C_{F}\left(\frac{1+z^{2}}{1-z+\epsilon\left(\kappa_{k}\right)}\right)
\end{aligned}
$$

where in the denominators the function $\epsilon$ appears,

$$
\epsilon\left(\kappa_{k}\right)=\frac{k^{\prime 2} n^{2}}{4(k \cdot n)^{2}} \simeq \frac{k^{2}}{k^{+2}},
$$

which arises here as a consequence of the $\partial_{r}^{2}$ term in the renormalization equations (95), after Fourier transforming with respect to $r^{-}=r^{0}-r^{3}$, the conjugate variable of $k^{+}$. It can be interpreted as a manifestation of the indeterminacy principle, which determines space-time uncertainty of the order of the cell size $\Delta r$ that is associated with the offshellness of the partons. The presence of $\epsilon$ effectively cuts off small-angle gluon emission when the emitted gluon is soft, i.e., when $z_{g}=1-z \rightarrow 0$, by modifying the free gluon propagator $\propto 1 / z_{g}$ to the form $1 /\left(z_{g}+\epsilon\right)$ when $k / k^{+} \sim 1$, that is, in branching processes with large space-time uncertainty. This ensures that the two daughter partons can be resolved as individual quanta only if they are separated sufficiently by $\Delta r \propto 1 / k$ in position space, in accord with the uncertainty principle. Note that $\epsilon$ can be neglected in the terms

\footnotetext{
${ }^{9}$ In (110) one could imagine, instead of the $\theta$ function $\theta\left(k^{2}-\mu^{2}\right), \quad$ a smeared-out probability distribution, e.g., $\propto \exp \left(-k^{2} / \mu^{2}\right)$, by choosing a more refined form for the function $\mathcal{T}$ under the time integral in Eqs. (103) and (104). The specific choice is ambiguous at this level of calculation (see Ref. [37] for details).
} 
$\propto 1 /\left(z_{g}+\epsilon\right)$ in Eq. (111) for energetic gluon emission $\left(z_{g} \rightarrow 1\right)$, but is essential in the soft regime $\left(z_{g} \rightarrow 0\right)$. The effect of $\epsilon$ has been shown $[27,28]$ to result in a natural regularization of the infrared-divergent behavior of the branching kernels [Eq. (111)], due to destructive gluon interference which becomes complete in the limit $z_{g} \rightarrow 0$.

As summarized in Appendix $\mathrm{C}$, the renormalization functions $\Delta_{f}$ are intimately related to the spectral densities $\mathcal{P}_{g}$ and $\mathcal{P}_{q}$ defined by Eqs. (61) and (85). In fact, since $\mathcal{P}_{g}$ $\left(\mathcal{P}_{q}\right)$ are defined as the trace over the difference of retarded and advanced proagators $D_{\mu \nu}^{R(A)}\left(S^{R(A)}\right)$, i.e.,

$$
\mathcal{P}_{g}=\sum_{f^{\prime}=g, q} \mathcal{P}_{g}^{f^{\prime}}=i \operatorname{Tr}\left[d^{\mu \nu}\left(D_{\mu \nu}^{R}-D_{\mu \nu}^{A}\right)\right]
$$

$$
\mathcal{P}_{q}=\sum_{f^{\prime}=g, q} \mathcal{P}_{q}^{f^{\prime}}=i \operatorname{Tr}\left[S^{R}-S^{A}\right]
$$

the solutions of $D_{\mu \nu}^{R(A)}$ and $S^{R(A)}$, and hence the solutions [Eq. (109)] of $\Delta_{g}$ and $\Delta_{q}$, determine the forms of $\mathcal{P}_{g}$ and $\mathcal{P}_{q}$, respectively. As has been investigated in detail by, e.g., Dokshitzer et al. [27], the Dyson-Schwinger integral representation of the densities $\mathcal{P}_{f}^{f^{\prime}}$ which is summed over in Eq. (113) follows from the integration over $\ln k^{2}\left(\ln p^{2}\right)$ of Eq. (103) and Eq. (104), respectively. These give (cf. Appendix C) then the self-energies $\Pi_{\mu \nu}^{R(A)}$ and $\Sigma^{R(A)}$, and hence $D_{\mu \nu}^{R(A)}$ and $S^{R(A)}$. Finally, by utilizing Eq. (113), one finds that the resulting solution of the densities $\mathcal{P}_{f}^{f^{\prime}}$ is given by

$$
\begin{aligned}
\mathcal{P}_{f}^{f^{\prime}}\left(r ; x, k^{2}\right)= & \delta_{f}^{f^{\prime}} \delta(1-x) \delta\left(k^{2}-\mu_{g q}^{2}\right) \Delta_{f}\left(r ; \mu_{g q}^{2}, k^{+2}\right) \\
& +\Delta_{f}\left(r, \mu_{g q}^{2}, k^{+2}\right) \sum_{f^{\prime \prime}} \int_{\mu_{g q}^{2}}^{k^{2}} \frac{d k^{\prime 2}}{k^{\prime 2}} \int_{0}^{1} d z\left\{A\left(r, k^{2}, z\right) \gamma_{f}^{f^{\prime \prime} f^{\prime}}(z, \epsilon) \mathcal{P}_{f^{\prime \prime}}^{f^{\prime}}\left(\frac{x}{z}, k^{\prime 2} ; k^{\prime+2}\right) \Delta_{f}^{-1}\left(r, \frac{k^{\prime 2}}{z}, k^{\prime+2}\right)\right\} .
\end{aligned}
$$

Comparing this expression with Eq. (85), $\mathcal{P}=\delta\left(k^{2}-\mu^{2}\right) \Delta \mathcal{P}_{\delta \mathcal{E}}+\Delta \mathcal{P}_{\Gamma}$, one sees now that the Sudakov form factor $\Delta_{f}$ together with the real emission and absorption probabilities $W_{f} \equiv \Sigma_{f^{\prime \prime}} \int d \ln k^{\prime 2} d z A \gamma_{f}^{f^{\prime \prime} f^{\prime}} \mathcal{P}_{f^{\prime \prime}}^{f^{\prime}}$ combine to play the role of the "wave function renormalization" part $\Delta \mathcal{P}_{\delta \mathcal{E}}$ and the dissipative part $\Delta \mathcal{P}_{\Gamma}$.

Equation (114) has a simple physical significance: The first term on the right hand side represents the probability to find a dressed parton of type $f$ in the cell around space-time point $r$ as a "classical" particle, i.e., without any other gluons or quarks present in its wave function or spectral density. In accord with the normalization [Eq. (98)], this means that it is propagating on shell with $k^{2}=\mu_{g q}^{2} \geqslant \mu^{2}(r)$, where $\mu(r)^{-1}$ is the resolution size of the cell as explained in Sec. III A. Its fraction of the cell's light-cone energy is $x=k^{+} / P^{+}(r)=1$. The probability for finding such a rare fluctuation is suppressed by the function $\Delta_{f}\left(r, k^{2}, k^{+2}\right)$, which becomes stronger with increasing gap between $\mu_{g q}^{2}$ and $k^{2}$. The second term on the right hand side corresponds then to the adjoint probability that the parton is actually a dressed parton with a substructure, described by the balance between real and virtual emission and absorption processes, while localized within the cell around $r$. It is obvious that the spectral densities of dressed partons, introduced in Sec. III C, are identical to the usual parton structure functions $\mathcal{P}_{f}$, i.e., the probability densities for finding a dressed parton $f$ in an intermediate state containing a number of bare partons as virtual and real fluctuations.

From Eq. (114) with Eq. (109), and using the representation (58) of the parton densities $F_{f}$ in terms of the parton structure functions $\mathcal{P}_{f}^{f^{\prime}}$, follows then the final form of the renormalization equations,

$$
\begin{aligned}
k^{2} \frac{\partial}{\partial k^{2}} F_{g}\left(r ; x, k^{2}\right)= & \int_{0}^{1} d z A\left(r ; k^{2}, z\right)\left\{\left[\frac{1}{z} F_{g}\left(r ; \frac{x}{z}, z k^{2}\right)\right.\right. \\
& \left.-\frac{1}{2} F_{g}\left(r ; x, k^{2}\right)\right] \Gamma_{g}^{g g}(z, \epsilon) \\
& +2 N_{f} F_{q}\left(r ; x, k^{2}\right) \Gamma_{q}^{g q}(z, \epsilon) \\
& \left.-N_{f} F_{g}\left(r ; x, k^{2}\right) \Gamma_{g}^{q q}(z, \epsilon)\right\}, \\
p^{2} \frac{\partial}{\partial p^{2}} F_{q}\left(r ; x, p^{2}\right)= & \int_{0}^{1} d z A\left(r ; p^{2}, z\right)\left\{\left[\frac{1}{z} F_{q}\left(r ; \frac{x}{z}, z p^{2}\right)\right.\right. \\
& \left.-F_{q}\left(r ; x, p^{2}\right)\right] \Gamma_{q}^{q g}(z, \epsilon) \\
& \left.+F_{g}\left(r ; x, p^{2}\right) \Gamma_{g}^{q q}(z, \epsilon)\right\},
\end{aligned}
$$

which are the space-time generalization of the DGLAP evolution equations [27] that govern the momentum dependence of the parton densities. The effective branching kernels $\Gamma_{f}^{f^{\prime} f^{\prime \prime}}[38]$ are related to the $\gamma_{f}^{f^{\prime \prime}}$ by

$$
\Gamma_{f}^{f^{\prime} f^{\prime \prime}}=\gamma_{f}^{f^{\prime} f^{\prime \prime}}\left(1-\frac{F_{f^{\prime}}}{F_{f^{\prime}} \pm 1}\right),
$$

where the upper (lower) sign in the term in parentheses is for gluons (quarks). It yields a suppression when the phasespace density $F_{g}$ or $F_{q}$ becomes large, so that the emission processes $f \rightarrow f^{\prime} f^{\prime \prime}$ have significant competition from absorption processes $f^{\prime} f^{\prime \prime} \rightarrow f$. In the limit $F_{f^{\prime}} \gg 1$, the detailed balance is established, in accord with the Bose-Einstein and 
Fermi-Dirac statistics of the gluon (quark) densities. For instance, in thermal equilibrium, $F_{g(q)}^{(\mathrm{eq})}=\left(e^{-E / T} \mp 1\right)^{-1}$, so that $\Gamma=\gamma\left(1-e^{E / T}\right)$, which tends to zero as the temperature $T$ becomes large.

Equations (115) are the main result of this section. They emerge as a direct consequence of the renormalization equations (67) in the short-distance regime of virtual and dissipative quantum fluctuations, and ensure unitarity conservation locally in each space-time cell. They embody the Heisenberg uncertainty principle, expressing the fact that it is impossible to localize soft partons in a given cell if their wavelength exceeds the cell size, which sets the resolution scale. Last but not least, they account for the balance between real emission and absorption processes that tends to increase (decrease) the effective real emission rate of gluons (quarks).

\section{E. Kinetic dynamics and transport equations}

With the dynamical structure of dressed partons quantitatively controlled by the above renormalization equations (115), one is now in the position to address the kinetic spacetime evolution of the multiparticle system in terms of statistical binary scatterings among these dressed partons. As explained in Sec. III A, in order to obtain quasiclassical transport equations for the phase-space distribution functions $F=\mathcal{N} \otimes \mathcal{P}$, two key conditions have to be met. First, as before, the maximal space-time extension of relevant quantum fluctuations, $\lambda_{c}=\mu_{g q}^{-1}$, is supposed to be smaller than the mean free path $\lambda_{\mathrm{mf}}$ between scatterings. Second, the typical four-momentum transfer $q_{\perp} \equiv \sqrt{\left|q^{2}\right|}$ in the scattering of any two partons is required to be larger than the inverse Compton wavelength $\lambda_{c}^{-1}=\mu_{g q}$. That is [cf. Eq. (49)],

$$
\lambda_{m f}>\mu_{g q}^{-1}, \quad q_{\perp}^{2}>\mu_{g q}^{2}=\lambda_{c}^{-2} .
$$

The first condition ensures that the quantum evolution, taken care of by the renormalization equations, can be factorized from the scattering processes. The second condition guarantees that the scattering is sufficiently hard, i.e., is of short range compared to the space-time extent of the scattering partons' intrinsic quantum motion, so that over the duration of the scattering, the dressed partons can be treated as "frozen" assemblies of bare particles that represent their instantaneous quantum state (the usual sudden approximation). These two conditions are equivalent to the factorization assumption of the well-established "QCD hard scattering picture', [39] for, e.g., high-energy hadron-hadron collisions, where the colliding hadrons are described as conglomerates of bare partons in terms of their structure functions. The relation of this hard scattering picture to the present approach is its adoption to multiple, internetted scattering processes in a system of stochastically colliding dressed partons, each of them represented by its own structure function, or spectral density.

The two requirements [Eq. (117)] are the crucial points, which allow one in the following to cast the kinetic evolution into simple, probabilistic Boltzmann-type equations, which, however, have to be solved self-consistently in conjunction with the renormalization equations (115). Here is the key difference from other formulations $[5,40,41]$ to include quantum effects in a quasiclassical treatment of transport phe- nomena, in which one has only one type of equation, a generalized Boltzmann equation, that contains a local, classical part and a nonlocal quantum contribution, containing the space-time history of memory effects. In the present approach, this is translated to stochastically occurring ("local'), hard parton-parton scatterings, linked with the causal quantum evolution between scatterings ("nonlocal'), accounting for renormalization and dissipation due to the previously occurred scatterings. The advantage here is, that while quantum effects are included in the multiparticle evolution, still a local (in space and time) picture can be maintained, where memory effects are embodied effectively in the dressed partons' structure function evolution.

To proceed, recall from Sec. III C, that the correlation functions $D_{\mu \nu}^{C}$ and $S^{C}$ are the quantities which are determined by the transport equations of the form (82) [explicitly given in Appendix B, Eqs. (B25) and (B26)]. On account of the presumed conditions (117), over kinetic space-time scales $\lambda_{\mathrm{mf}}>\lambda_{c}$, the quantum motion decouples, so that the correlation functions are determined by the collisional selfenergies $\Pi_{\mu \nu}^{C}$ and $\Sigma^{C}$, in conjunction with the real parts of the retarded and advanced functions [cf. Eq. (82)]. This means that the collisional self-energies are to be evaluated with the renormalized propagators [Eq. (96)] and vertices [Eq. (100)] which were obtained from the retarded and advanced self-energies before.

Noting that from Eq. (77) one has

$$
D_{\mu \nu}^{C}=D_{\mu \nu}^{>}+D_{\mu \nu}^{<}, \quad S^{C}=S^{>}+S^{<}
$$

the transport equations (68) now read (cf. Appendix B)

$$
\begin{aligned}
k \cdot \partial_{r} D_{a b}^{\mu \nu \gtrless}(r, k)= & -\frac{i}{2}\left(\Pi^{\gtrless} D^{A}+\Pi^{R} D^{\gtrless}-D^{\gtrless} \Pi^{A}\right. \\
& \left.-D^{R} \Pi^{\gtrless}\right)_{a b}^{\mu \nu}, \\
i\left\{\gamma \cdot \partial_{r}, S_{i j}^{\gtrless}(r, p)\right\}_{+}= & -\left(\left[\gamma \cdot p, S^{\gtrless}\right]_{-}\right)_{i j} \\
& +\left(\Sigma^{\gtrless} S^{A}+\Sigma^{R} S^{\gtrless}-S^{\gtrless} \Sigma^{A}-S^{R} \Sigma^{\gtrless}\right)_{i j},
\end{aligned}
$$

which can be rewritten as

$$
\begin{aligned}
k \cdot \partial_{r} D_{a b}^{\mu \nu \gtrless}(r, k)= & -\frac{1}{2}\left(\left\{\Pi^{>}(r, k), D^{<}(r, k)\right\}_{+}\right. \\
& \left.-\left\{\Pi^{<}(r, k), D^{>}(r, k)\right\}_{+}\right)_{a b}^{\mu \nu}, \\
p \cdot \partial_{r} S_{i j}^{\gtrless}(r, p)= & \frac{1}{2}\left(\left\{\Sigma^{>}(r, p), S^{<}(r, p)\right\}_{+}\right. \\
& \left.-\left\{\Sigma^{<}(r, p), S^{>}(r, p)\right\}_{+}\right)_{i j} .
\end{aligned}
$$

These equations correspond to what is usually termed the quasiparticle approximation. In the cellular space-time picture, the characteristics of the statistical-kinetic evolution of the system are, per design, insensitive to the localized fluctuations associated with short-distance quantum dynamics inside a space-time cell. To stress it more precisely, the space-time variation can be considered homogeneous over the range of the Compton wavelength $\lambda_{c}=\mu_{g q}^{-1} \leqslant \mu^{-1}(r)$, so that $\left|G^{C}(r, k)\right| \gg\left|\lambda_{c}^{2} \partial_{r}^{2} G^{C}(r, p)\right|$ and the derivatives with respect to $r$ on the left hand side of the original Eqs. (67) and 
(68) may be omitted. In the present context, it emerges as the logical consequence that the partons can be described on kinetic space-time scales as quasiparticles, with the underlying quantum motion effectively accounted for in the renormalized propagators and vertices.
The self-energies $\Pi_{\mu \nu}^{\gtrless}$ and $\Sigma^{\gtrless}$ are obtained from the general expressions (42) and (43), respectively. The lowestorder nonvanishing contributions are the two-loop diagrams shown in Fig. 10, which are proportional to $\hbar$ and $O\left(g_{s}^{4}\right)$. In terms of the renormalized correlators $D^{\gtrless}$ and $S^{\gtrless}$ one finds

$$
\begin{aligned}
& \Pi_{a b}^{\sigma \tau \gtrless}(r, k)=\frac{g_{s}^{4}}{2} \int \frac{d^{4} k^{\prime}}{(2 \pi)^{4} i} \frac{d^{4} q}{(2 \pi)^{4} i} f_{a a^{\prime} c} \lambda_{\mu \mu^{\prime} \sigma}\left(-k, k-k^{\prime}, k^{\prime}\right) D_{c c^{\prime}}^{\sigma \sigma^{\prime} \gtrless}\left(r, k^{\prime}\right) f_{c^{\prime} f e} \lambda_{\sigma^{\prime} \rho \lambda}\left(-k^{\prime},-q, q+k^{\prime}\right) \\
& \times D_{f f^{\prime}}^{\rho \rho^{\prime} \gtrless}(r,-q) f_{e^{\prime} f^{\prime \prime} d^{\prime}} \lambda_{\lambda^{\prime} \rho^{\prime} \tau^{\prime}}\left(-q-k^{\prime}, q, k^{\prime}\right) D_{d d^{\prime}}^{\tau^{\prime} \tau \gtrless}\left(r, q+k^{\prime}\right) f_{d b^{\prime} b} \lambda_{\tau \nu^{\prime} \nu}\left(-k^{\prime},-k+k^{\prime}, k\right) D_{a^{\prime} b^{\prime}}^{\mu^{\prime} \nu^{\prime} \gtrless}\left(r, k-k^{\prime}\right) \\
& +\frac{g_{s}^{4}}{6} \int \frac{d^{4} k^{\prime}}{(2 \pi)^{4} i} \frac{d^{4} q}{(2 \pi)^{4} i} v_{a c^{\prime} a^{\prime} c}^{\mu \sigma^{\prime} \mu^{\prime} \sigma}\left(-k, q^{\prime}, k-q-q^{\prime}, q\right) D_{c d}^{\sigma \tau \gtrless}(r, q) D_{c^{\prime} d^{\prime}}^{\sigma^{\prime} \tau^{\prime} \gtrless}\left(r, q^{\prime}\right) v_{d b^{\prime} d^{\prime} b}^{\tau \nu^{\prime} \tau^{\prime} \nu}\left(-q,-k+q+q^{\prime},-q^{\prime}, k\right) \\
& \times D_{a^{\prime} b^{\prime}}^{\mu^{\prime} \nu^{\prime}}\left(r, k-q-q^{\prime}\right)+g_{s}^{4} N_{f} \int \frac{d^{4} k^{\prime}}{(2 \pi)^{4} i} \frac{d^{4} q}{(2 \pi)^{4} i} f_{a a^{\prime} c} \lambda_{\mu \mu^{\prime} \sigma}\left(-k, k-k^{\prime}, k^{\prime}\right) D_{c c^{\prime}}^{\sigma \sigma^{\prime} \gtrless}\left(r, k^{\prime}\right) \gamma_{\sigma^{\prime}} T_{l n}^{c^{\prime}} S_{n n^{\prime}}^{\gtrless}(r,-q) \\
& \times \gamma_{\tau^{\prime}} T_{n^{\prime} l^{\prime}}^{d^{\prime}} S_{l^{\prime} l}^{\gtrless}\left(r, q+k^{\prime}\right) D_{d^{\prime} d}^{\tau^{\prime} \tau}\left(r, k^{\prime}\right) f_{d b^{\prime} b} \lambda_{\tau \nu^{\prime} \nu}\left(-k^{\prime},-k+k^{\prime}, k\right) D_{a^{\prime} b^{\prime}}^{\mu^{\prime} \nu^{\prime} \gtrless}\left(r, k-k^{\prime}\right)-g_{s}^{4} 2 N_{f} \int \frac{d^{4} k^{\prime}}{(2 \pi)^{4} i} \frac{d^{4} q}{(2 \pi)^{4} i} \\
& \times \gamma_{\mu} T_{l i}^{a} S_{l n}^{\gtrless}\left(r, k-k^{\prime}\right) \gamma_{\nu} T_{j n}^{b} S_{j^{\prime} j}^{\gtrless}\left(r, k^{\prime}\right) \gamma_{\tau^{\prime}} T_{n^{\prime} j^{\prime}}^{d} S_{l^{\prime} n^{\prime}}^{\gtrless}\left(r, k^{\prime}-q\right) \gamma_{\sigma^{\prime}} T_{i^{\prime} l^{\prime}}^{c} S_{i i^{\prime}}^{\gtrless}\left(r, k^{\prime}\right) D_{c d}^{\sigma \tau \gtrless}(r, q), \\
& \Sigma_{i j}^{\gtrless}(r, p)=-\frac{g_{s}^{4}}{2} \int \frac{d^{4} k^{\prime}}{(2 \pi)^{4} i} \frac{d^{4} q}{(2 \pi)^{4} i} \gamma_{\sigma} T_{i i^{\prime}}^{c} D_{c c^{\prime}}^{\sigma \sigma^{\prime} \gtrless}\left(r, k^{\prime}\right) f_{c^{\prime} f e} \lambda_{\sigma^{\prime} \rho \lambda}\left(-k^{\prime},-q, q+k^{\prime}\right) D_{f f^{\prime}}^{\rho \rho^{\prime} \gtrless}(r,-q) \\
& \times f_{e^{\prime} f^{\prime \prime} d^{\prime}} \lambda_{\lambda^{\prime} \rho^{\prime} \tau^{\prime}}\left(-q-k^{\prime}, q, k^{\prime}\right) D_{d d^{\prime}}^{\tau^{\prime} \tau}\left(r, q+k^{\prime}\right) \gamma_{\tau} T_{j^{\prime} j^{\prime}}^{d} S_{i^{\prime} j^{\prime}}^{\gtrless}\left(r, p-k^{\prime}\right) \\
& -g_{s}^{4} N_{f} \int \frac{d^{4} k^{\prime}}{(2 \pi)^{4} i} \frac{d^{4} q}{(2 \pi)^{4} i} \gamma_{\sigma} T_{i i^{\prime}}^{c} D_{c c^{\prime}}^{\sigma \sigma^{\prime}} \gtrless\left(r, k^{\prime}\right) S_{n n^{\prime}}^{\gtrless}(r,-q) \gamma_{\sigma^{\prime}} T_{l n}^{c^{\prime}} S_{l^{\prime} l}^{\gtrless}\left(r, q+k^{\prime}\right) \gamma_{\tau^{\prime}} T_{n^{\prime} l^{\prime}}^{d^{\prime}} D_{d^{\prime} d}^{\tau^{\prime} \tau \gtrless}\left(r, k^{\prime}\right) \\
& \times \gamma_{\tau} T_{j^{\prime} j^{\prime}}^{d} S_{i^{\prime} j^{\prime}}^{\gtrless}\left(r, p-k^{\prime}\right)+g_{s}^{4} \int \frac{d^{4} k^{\prime}}{(2 \pi)^{4} i} \frac{d^{4} q}{(2 \pi)^{4} i} \gamma_{\sigma} T_{i l}^{c} S_{l l^{\prime}}^{\gtrless}\left(r, p-k^{\prime}\right) D_{e e^{\prime}}^{\lambda \lambda^{\prime} \gtrless}(r, q) \\
& \times \gamma_{\lambda} T_{l^{\prime} m}^{e} S_{m m^{\prime}}^{\gtrless}\left(r, p-k^{\prime}-q\right) \gamma_{\tau} T_{n j}^{d} D_{c d}^{\sigma \tau \gtrless}\left(r, k^{\prime}\right),
\end{aligned}
$$

where $\lambda^{\mu \rho \nu}\left(p_{1}, p_{2}, p_{3}\right)$ and $v_{a b c d}^{\mu \sigma \tau v}\left(p_{1}, p_{2}, p_{3}, p_{4}\right)$ are the usual three-gluon and four-gluon vertices [cf. Appendix D, Eq. (D27)]. The correlation functions $D_{\mu \nu}^{\gtrless}$ and $S^{\gtrless}$ are related to the phase-space densities $F_{g}$ and $F_{q}$ : by employing Eq. (92) in conjunction with the identities Eqs. (74)-(77), one obtains

$$
\begin{aligned}
D_{\mu \nu}^{\gtrless}(r, k)= & -2 \pi i\left(-d_{\mu \nu}(k)\right)\left[\theta\left( \pm k^{0}\right)+F_{g}(r, k)\right] \\
\times & \delta\left(k^{2}-\mathcal{M}_{g}^{2}(r, k)\right), \\
S^{\gtrless}(r, p)= & -2 \pi i(\gamma \cdot p)\left[\theta\left( \pm p^{0}\right)-F_{q}(r, p)\right] \\
& \times \delta\left(p^{2}-\mathcal{M}_{q}^{2}(r, p)\right),
\end{aligned}
$$

where the signs $+(-)$ refer to $>(<) .{ }^{10}$ As repeatedly stressed, the densities $F$ are the distributions of dressed par-

\footnotetext{
${ }^{10}$ It must be mentioned that Eq. (123) assumes a spin-symmetric form for the quark-antiquark spinor products, which means a neglect of spin-polarization effects. As shown by Elze et al. [16], in general the quark phase-space distribution does require at least an $8 \times 8$ matrix representation.
}

tons, with their substructure represented in terms of corresponding assemblies of bare partons, that satisfy the condition (56). Therefore the functions $F$ can also be interpreted to measure the number of bare partons with dynamical invariant masses $k^{2} \geqslant \mu_{g q}^{2}$. Consequently, a binary collision of two dressed partons can be described in terms of the above "hard scattering picture", as a scattering of two bare partons, one out of each assembly, picked statistically from the instantaneous quantum state of the two dressed partons, as given by their structure functions or spectral densities. The fourmomentum transfer $q_{\perp}^{2}$ sets hereby the probing scale, so that $k^{2} \approx q_{\perp}^{2}$. Therefore the energy spectra of partons emerging from these scatterings are-using Eq. (88)_determined by

$$
\begin{aligned}
& \omega \equiv \pm k^{0}(r, k)=\omega_{(0)}\left(1+\frac{\mathcal{M}_{g}^{2}\left(r, k^{+}, k^{2}\right)}{2 \omega_{(0)}}\right), \\
& E \equiv \pm p^{0}(r, p)=E_{(0)}\left(1+\frac{\mathcal{M}_{q}^{2}\left(r, p^{+}, p^{2}\right)}{2 E_{(0)}}\right),
\end{aligned}
$$




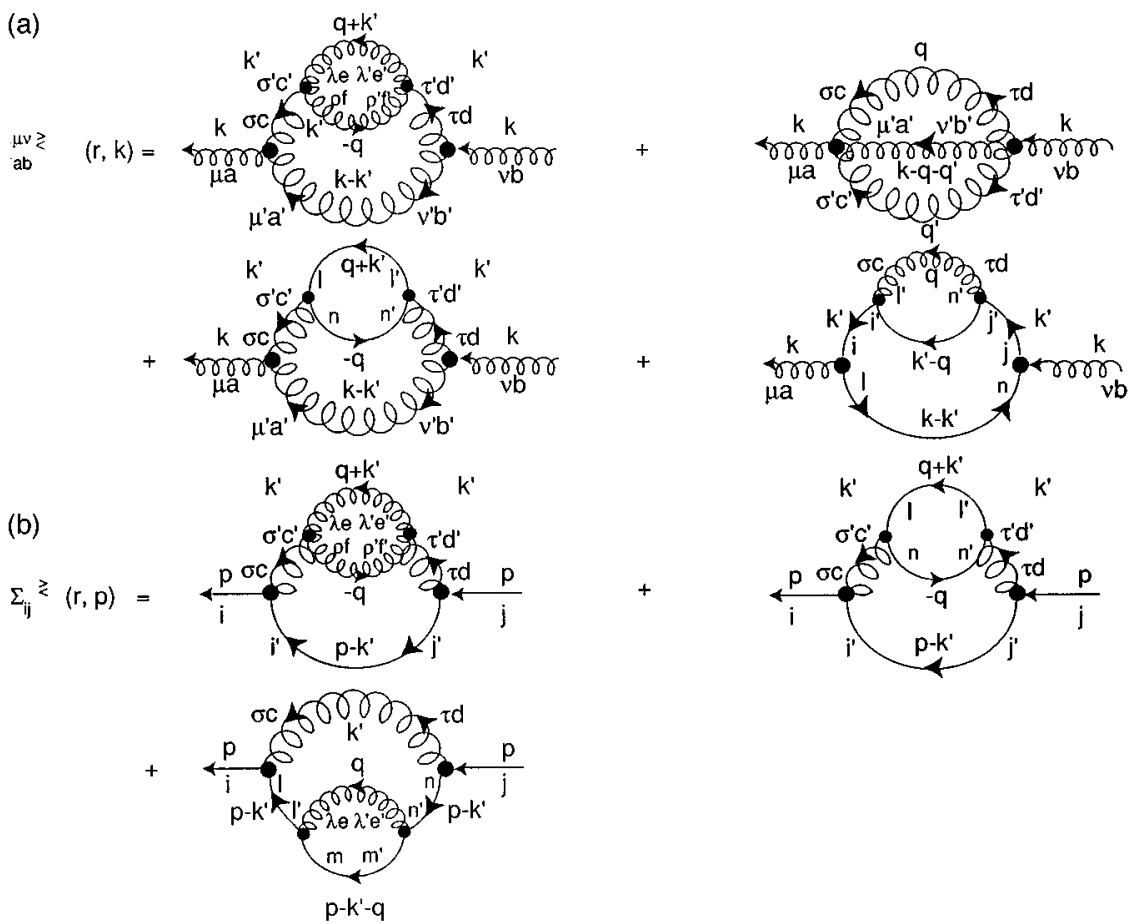

FIG. 10. The "collisional" self-energies in two-loop approximation, Eqs. (121) and (122): (a) the contributions to the gluon correlation functions $\Pi_{\mu \nu}^{\gtrless}$ and (b) the contributions to the quark correlation functions $\Sigma \gtrless$. where $k^{0}=1 / 2\left(k^{+}+k^{-}\right) \simeq k^{+} / 2, \quad p^{0} \simeq p^{+} / 2, \quad$ and $\quad \omega_{(0)}$ $= \pm \sqrt{\vec{k}^{2}+\mu_{g q}^{2}}, E_{(0)}= \pm \sqrt{\vec{p}^{2}+\mu_{g q}^{2}}$. Accordingly, one can now write

$\omega= \pm \sqrt{\vec{k}^{2}+q_{\perp}^{2}} \theta\left(q_{\perp}^{2}-\mu_{g q}^{2}\right), \quad E= \pm \sqrt{\vec{p}^{2}+q_{\perp}^{2}} \theta\left(q_{\perp}^{2}-\mu_{g q}^{2}\right)$,

and

$$
\begin{aligned}
F_{g}(r, k) \delta\left(k^{2}-q_{\perp}^{2}\right)= & \frac{1}{2 \omega}\left[F_{g}(r, \vec{k}) \delta\left(k^{0}-\omega\right)\right. \\
& \left.+F_{g}(r,-\vec{k}) \delta\left(k^{0}+\omega\right)\right], \\
F_{q}(r, p) \delta\left(p^{2}-q_{\perp}^{2}\right)= & \frac{1}{2 E}\left[F_{q}(r, \vec{p}) \delta\left(p^{0}-E\right)\right. \\
& \left.+\bar{F}_{q}(r,-\vec{p}) \delta\left(p^{0}+E\right)\right],
\end{aligned}
$$

which exhibits explicitly the particle-antiparticle character of the phase-space densities. In particular, $F_{q}(r, \vec{p})$ denotes the quark distribution and $\bar{F}_{q}(r,-\vec{p})$ the antiquark distribution.

Using the representations (123) for $D_{\mu \nu}^{\gtrless}, S^{\gtrless}$ in the selfenergies Eqs. (122) and (121), and substituting into Eqs. (120) gives the final form of the transport equations in the kinetic regime (cf. Appendix D),

$$
k \cdot \partial_{r} F_{g}(r, k)=\mathcal{I}_{g}(r, k), \quad p \cdot \partial_{r} F_{q}(r, p)=\mathcal{I}_{q}(r, p),
$$

where

$$
\begin{aligned}
\mathcal{I}_{g}(r, k)= & \frac{1}{2}\left\{\hat{\Pi}^{>}(r, k), F_{g}(r, k)\right\}_{+} \\
& -\frac{1}{2}\left\{\hat{\Pi}^{<}(r, k), F_{g}(r, k)+1\right\}_{+},
\end{aligned}
$$

$$
\begin{aligned}
\mathcal{I}_{q}(r, p)= & \frac{1}{2}\left\{\hat{\Sigma}^{>}(r, p), F_{q}(r, p)\right\}_{+} \\
& -\frac{1}{2}\left\{\hat{\Sigma}^{<}(r, p), F_{q}(r, p)-1\right\}_{+},
\end{aligned}
$$

and the careted self-energy functions $\hat{\Pi}$ and $\hat{\Sigma}$ stand for

$$
\begin{gathered}
\hat{\Pi}^{\gtrless}(r, k)=\frac{1}{2 i} \sum_{s=1,2} \varepsilon^{\mu}(k, s) \varepsilon^{\nu *}(k, s) \Pi_{\mu \nu}^{\gtrless}(r, k), \\
\hat{\Sigma}^{\gtrless}(r, p)= \\
\frac{1}{2 i} \sum_{s=1,2}\left[\bar{u}(p, s) \Sigma^{\gtrless}(r, p) u(p, s)\right. \\
\left.+\bar{v}(p, s) \Sigma^{\gtrless}(r,-p) v(p, s)\right] .
\end{gathered}
$$

The collision terms $\mathcal{I}_{g}$ and $\mathcal{I}_{q}$ on the right hand side of Eq. (127) describe the balance of gain and loss of partons in a phase-space element $d^{3} r d^{4} k$, or $d^{3} d^{4} p$, within a time slice around $r_{0}$. Their explicit form is obtained as explained in Appendix D, and emerges as the result of applying the usual cutting rules [42] to the self-energies Eqs. (121) and (122) and averaging (summing) over initial (final) spin and color degrees of freedom (see Fig. 11). The resulting structure of the collision terms is

$\mathcal{I}_{a}\left(r, p_{1}\right) \equiv \sum_{b c d}\left(-\mathcal{I}_{c d \rightarrow a b}^{(\text {loss })}\left(p_{1}, r\right)+\mathcal{I}_{a b \rightarrow c d}^{(\text {gain })}\left(p_{1}, r\right)\right]$

$$
=-\sum_{b c d} C_{a b} C_{c d} \int \frac{d^{3} p_{2}}{(2 \pi)^{3} 2 E_{2}} \int \frac{d^{3} p_{3}}{(2 \pi)^{3} 2 E_{3}}
$$

$$
\begin{aligned}
& \times \int \frac{d^{3} p_{4}}{(2 \pi)^{3} 2 E_{4}}(2 \pi)^{4} \delta^{4}\left(p_{1}+p_{2}-p_{3}-p_{4}\right) \\
& \times\left\{F_{a}(1) F_{b}(2)|\mathcal{M}(a b \rightarrow c d)|^{2} \theta\left(q_{\perp}^{2}-\mu_{g q}^{2}\right)\right.
\end{aligned}
$$



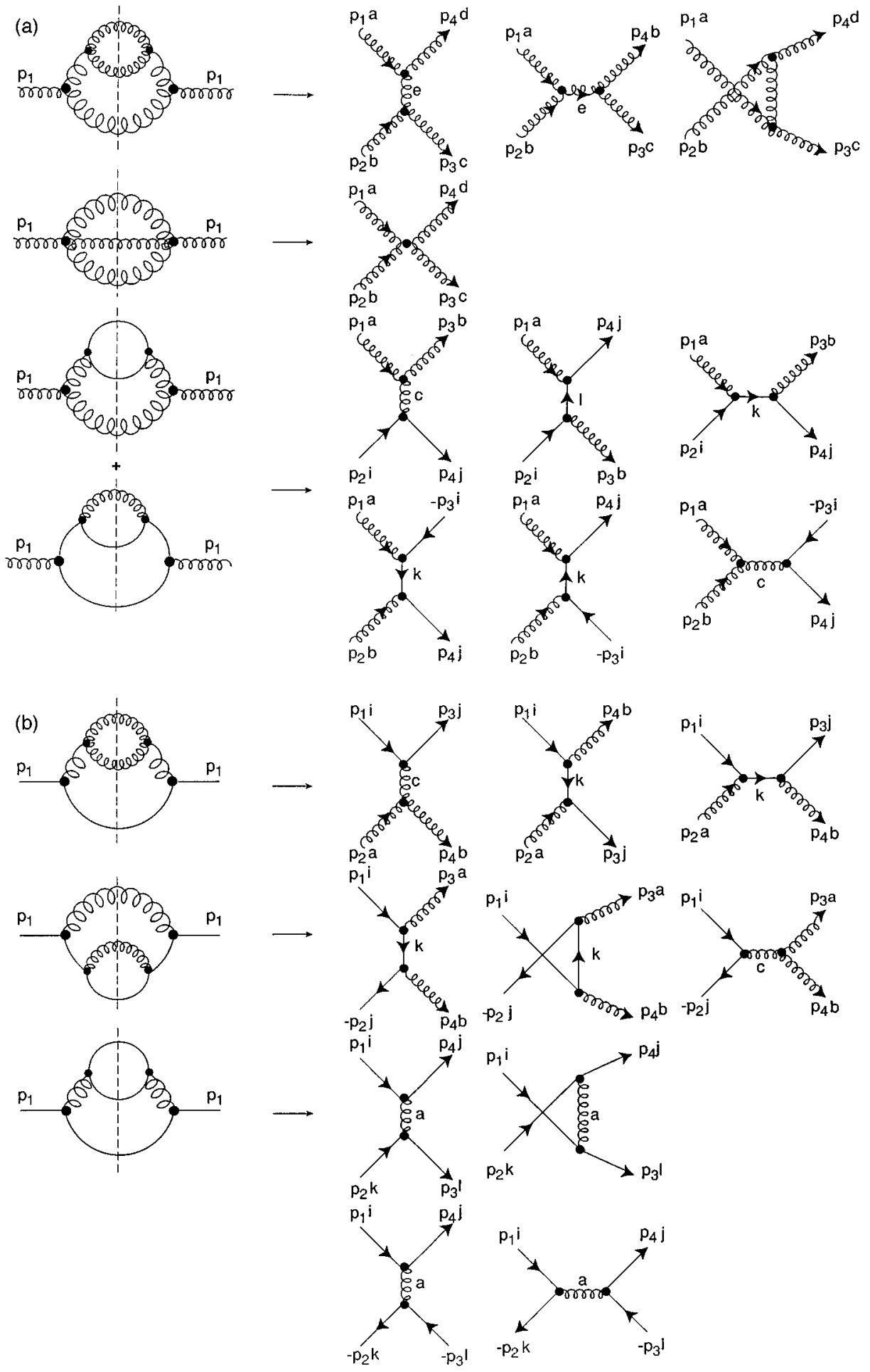

FIG. 11. Cutting the "collisional" two-loop self-energies gives the different binary $2 \rightarrow 2$ collision processes, namely, (a) the gluon terms $g g \leftrightarrow g g, g q \leftrightarrow g q, g g \leftrightarrow q \bar{q}$, and (b) the quark terms, $q g \leftrightarrow q g, q \bar{q} \leftrightarrow q \bar{q}, q q \leftrightarrow q q$.

$$
\begin{aligned}
& \times\left[1 \pm F_{c}(3)\right]\left[1 \pm F_{d}(4)\right] \\
& -\left[1 \pm F_{a}(1)\right]\left[1 \pm F_{b}(2)\right]|\mathcal{M}(c d \rightarrow a b)|^{2} \\
& \left.\times \theta\left(q_{\perp}^{2}-\mu_{g q}^{2}\right) F_{c}(3) F_{d}(4)\right\} .
\end{aligned}
$$

Here the notation is $F_{f}(i) \equiv F_{f}\left(r, p_{i}\right)$ for the distribution functions of the parton species $f=g, q, \bar{q}$ with four-momenta $p_{i}=p, p_{2}, p_{3}, p_{4}$ at space-time point $r=\left(r^{0}, \vec{r}\right)$. The structure of the collision terms in conjunction with Eqs. (127) is such that the squared matrix elements for the various scattering processes $12 \rightarrow 34$ (depicted in Fig. 11, and explicitly given in Appendix D) are weighted by a distribution function $F_{f}(i)$ for each of the partons coming into the vertex and a factor $\left[1 \pm F_{f^{\prime}}(j)\right]$ for each of the outgoing ones. The + sign is for gluons so that $\left(1+F_{g}\right)$ results in a Bose enhancement, 
(a)
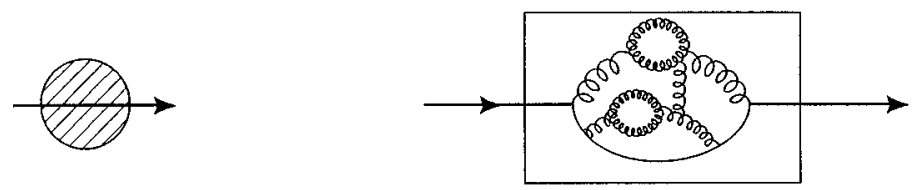

(b)
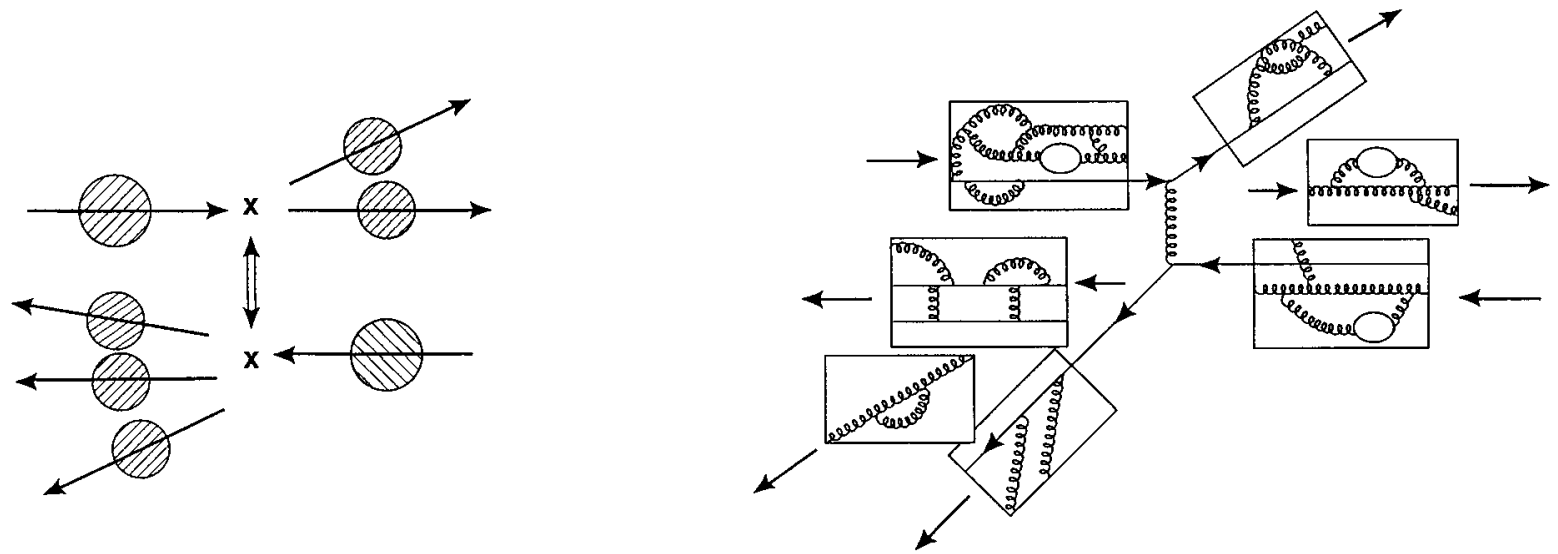

FIG. 12. Illustration of the "hard scattering picture," for the evolution of a multiparton system on the basis of the coupled renormalization and transport equations. (a) A dressed parton is described as a quasiparticle with a dynamical substructure, corresponding to an instantaneous state consisting of a number of bare gluons and quarks (its radiative cloud). The underlying quantum fluctuations are embodied in the spectral densities, or parton structure functions, which are determined by the renormalization equations. (b) A binary collision between two dressed partons is described as a statistically occurring "hard scattering," determined by the local density of dressed partons, and convoluted with their spectral densities at the "hard scattering scale" of the order of the momentum transfer. This is described by the transport equations.

and the - sign refers to quarks and antiquarks with $\left(1-F_{q}\right)$ indicating Pauli blocking. This is a direct consequence of the quantum-statistical difference between the gluon and quark propagators [Eqs. (75) and (78)]. The factors $C_{a b}\left(C_{c d}\right)$ in front account for the identical particle effect, if incoming (outgoing) partons are indistinguishable.

Equations (127) are the essential result of this section. These Boltzmann-type equations are the final form of the transport equations for the dressed partons with phase-space densities $F$. The equations have a drift term on the left hand side and a collision term on the right hand side, which balances the various processes by which a dressed parton may be gained or lost in a phase-space element $d^{3} r d^{4} p$ around time $r^{0} \pm \Delta r / 2=r^{0} \pm 1 /(2 \mu)$. They describe the dynamics of the multiparton system on kinetic scales, due to statistical, binary collisions, in which dressed partons appear as quasiparticles with a dynamical substructure, which is described in terms of probabilities to find a parton as a state consisting of a number of bare gluons and quarks of virtualities $k^{2}>\mu_{g q}^{2}$. These the underlying quantum fluctuations are embodied in $F=\mathcal{N} \otimes \mathcal{P}$ through the spectral density, or parton structure function, $\mathcal{P}$ and are determined by the renormalization equations (115). A scattering between two dressed partons is therefore described as a "hard scattering" determined by the probabilities of finding in each of them a hard fluctuation with $k^{2} \simeq q_{\perp}^{2}>\mu_{g q}^{2}$ of the order of the momentum transfer that sets the probing scale. This is expressed by the collision term on the right hand side, in which the products of $F$ 's involve the convolution of spectral densities $\mathcal{P}$, weighted with the squared matrix elements. A graphical il- lustration of this is shown in Fig. 12.

\section{F. A Monte Carlo calculation scheme}

A practical calculation scheme to compute the evolution of a multiparton system as governed by the coupled renormalization and transport equations may be outlined with the following concrete example. Consider the collision of two large nuclei with mass number $A \gg 1$ at ultrarelativistic center-of-mass energy. Before their contact upon collision, the approaching nuclei appear as two highly Lorentzcontracted disks of coherently bound gluons and quarks with a coherence length $L_{0}=1 / \mu_{0}$, where $\mu_{0} \simeq g_{s} \rho_{\perp}$ with $\rho_{\perp}=N_{g q} /\left(\pi R_{A}^{2}\right)$ sets the scale of the typical spacelike parton virtuality, and hence $L_{0}$ their characteristic transverse size. McLerran and Venugopalan [25] have shown that if $A$ is sufficiently large, the associated primeval parton distribution of the nuclei before and shortly after the collision can be calculated nonperturbatively from first principles in terms of coherent quantum fields. Their conclusion is that, as long as the very early generation of this parton matter distribution has $\Delta p \Delta r \sim 1$, it cannot be described by a kinetic particle picture, which requires $\Delta p \Delta r \gg 1$. However, after a time $t_{0} \simeq 1 / \mu_{0}$ past the nuclear contact, the parton matter has gone through a decoherence stage, so that the latter condition is satisfied, and a kinetic description can be matched to the complex coherent evolution of the primeval matter. In other words, at time $t_{0}$, one may proceed with a probabilistic description of the parton dynamics in terms of the interplay 
between coherent radiative evolution and incoherent binary interactions, as suggested in the present work.

At time $t_{0}$, one starts from the initial multiparton state, and the subsequent time evolution of the partons' phasespace densities $F_{g q}\left(t, \vec{r}, p^{2}, \vec{p}\right)$ may be calculated by a Monte Carlo procedure, using the advocated discretization of spacetime with four-dimensional cells of size $\Delta r=\Delta t \Delta^{3} r \simeq \mu^{-4}$.

(i) The first step consists in evaluating, from the collision kernel of the transport equations (127), the probabilities of scatterings among the initial partons within the time slice $\Delta t_{0}=\mu^{-1}\left(t_{0}\right) \equiv \mu_{0}^{-1}$ between $t_{0}$ and $t_{0}+\Delta t_{0}$ for each cell centered around $\vec{r}$. The essential condition (117) provides the possibility of treating the scattering among partons incoherently, and requires that the impact parameter $b_{a b}$ of any two scattering candidates $a$ and $b$ must satisfy $b_{a b}<\mu_{0}^{-1}$, implying for the momentum transfer of the scattering $q_{a b \perp}^{2}>\mu_{0}^{2}$, where $\mu_{0}^{2}$ is the initial virtuality of the partons at $t_{0}$, set by the coherence length of the colliding nuclei. The primary parton scatterings $a+b$ that occur within $\Delta t_{0}$ subject to this condition change the phase-space occupation of partons at $t_{1}=t_{0}+\Delta t_{0}$ in two ways: on the one hand their spatial profile is altered due to gain and loss of deflected scatterers $a^{\prime}$ and $b^{\prime}$ in the spatial cells, and on the other hand, the virtualities are reset from $k_{a}^{2}=k_{b}^{2}=\mu_{0}^{2}$ to $k_{a}^{\prime 2}=k_{b}^{\prime 2}=q_{a b \perp}^{2}>\mu_{0}^{2}$.

(ii) In the next step one must now calculate the quantum fluctuations, virtual plus real emission and absorption processes, that are triggered by the primary scatterings and the change of virtualities from $k^{2}$ to $k^{\prime 2}$. That is, the parton structure functions need to be evolved within $\Delta t_{0}$ according to the renormalization equations (115), which account for the associated renormalization and dissipation. One then obtains a spatial profile of new dressed partons that defines the initial condition for the further evolution, starting at $t_{1}$. The procedure repeats, as before at $t_{0}$, by evaluating the scattering probabilities in the next time slice $\Delta t_{1}=\mu^{-1}\left(t_{1}\right)$ between $t_{1}$ and $t_{1}+\Delta t_{1}$, now, however, subject to the modified incoherence condition that for any scattering of partons $a$ and $b$ their impact parameter must be $b_{a b}<\max \left(k_{a}^{2}, k_{b}^{2}\right)^{-1 / 2}$, i.e., $q_{a b \perp}^{2}>\max \left(k_{a}^{2}, k_{b}^{2}\right)$.

It is important to realize that the condition (117) of a well-defined separation between quantum and kinetic scales imposes the crucial incoherence requirement for binary scatterings, and allows a "hard scattering" picture, in which the quantum evolution and hard scattering of evolving quanta factorize. The condition defines the range of validity for a probabilistic description in terms of incoherently scattering particles, and is essentially the uncertainty principle: the quasiclassical picture holds only if the scattering partons may be treated as clearly distinguishable, incoherent quasiparticles of size $1 / \sqrt{k^{2}}$, meaning that at least a "formation time" of $t_{k} \simeq 1 / \sqrt{k^{2}}$ must have passed since their previous scattering, during which their quantum structure evolves with virtuality $k^{2}$. A concrete example of this scheme will be presented elsewhere.

\section{CONCLUDING REMARKS}

In this paper I have attempted to lay out a foundation to obtain from the fundamental quantum field theoretical principles of QCD a self-consistent kinetic description for the evolution of a high-energy system of self- and mutually interacting gluons and quarks. The main result is a set of two distinct but coupled equations that govern the time evolution of the gluon and quark Wigner functions, the quantum analogues of the classical phase-space densities.

(i) A renormalization equation, which describes the momentum dependence of short-distance quantum fluctuations due to the partons' self-interactions. It defines the state of a dressed parton as a quasiparticle with a renormalized mass and a decay width, corresponding to virtual and real emission and absorption processes. The solution of this equation describes, locally in space-time, the spectral density in terms of bare partons that are associated with the quantum substructure of the state of a dressed parton, and determines the partons' structure functions as well as their dynamical mass spectrum.

(ii) A transport equation, which describes the space-time evolution of the dressed partons in the kinetic quasiparticle regime by means of mutual binary collisions. It determines the time dependence of the change both of the spatial density and the energy-momentum distribution of dressed partons due to elastic and inelastic collisions. Accordingly, it not only redistributes the partons in space, but also modifies their momentum spectrum and virtualities, which feed back into the renormalization equations.

Loosely speaking, the renormalization equation defines the state of dessed partons, whereas the transport equation governs the occupation of these states. The self-consistent solution of the equations provides the means to trace the dynamical development of the multiparton system in real time and full seven-dimensional phase space $d^{3} r d^{3} p d p^{2}$, spanned by position, momentum, and invariant virtuality. It suggests a probabilistic, causal description, which is predestined for numerical evaluation by using Monte Carlo simulation techniques.

The essential steps that lead to this kinetic framework may be summarized as follows:

(a) the path integral quantization of the classical action, using the CTP formalism with in-in boundary conditions, including initial state correlations at time $t_{0}$ described by the density matrix $\hat{\rho}\left(t_{0}\right)$;

(b) the two-point source approximation, which allows one to rewrite the CTP path integral as the generating functional for a possible color background field, and for the two-point gluon and quark Green functions defined on the closed time path between $t_{0}$ and $t_{\infty}$;

(c) the derivation of the self-consistent equations of motion for mean field (Ginzburg-Landau equation) and Green functions (Dyson-Schwinger equations);

(d) the transition to kinetic theory by imposing the physics-motivated well-defined separation between the quantum scale that specifies the range of short-distance quantum fluctuations and the kinetic scale that characterizes the range of interparticle correlations and stochastic binary interactions;

(e) the conversion of the Dyson-Schwinger equations for the Green functions to the set of renormalization and transport equations for the corresponding Wigner functions, on the basis of the separation of quantum and kinetic scales and a cellular space-time picture;

(f) the calculation of the spectral density of bare partons, 
locally within the cells, from the renormalization equations, which define the state of dressed partons in terms of their substructure, and the evaluation of the collision kernel of the transport equations, which determine the statistical occurrence of scattering processes among these dressed partons.

This quantum-kinetic framework may be extended in a straightforward manner to include effects of a color background field, or gluonic mean field, that acts as a classical background medium in which the partonic quanta evolve (e.g., in a QCD plasma, where it may be generated due to the bulk dynamics of soft gluon modes). This option has not been considered in the present paper; however, the framework incorporates this possibility by considering a nonvanishing $\widetilde{A^{\mu}}$ instead of setting it to zero as in Sec. III. The inclusion of such a mean field would extend the set of renormalization and transport equations for the partons' Wigner functions, by coupling to the Ginzburg-Landau equation for the mean field. Qualitatively, the effect would be twofold: first, the poles of the Wigner functions would be shifted by a mean-field-generated mass $\mu\left(\widetilde{A}^{\mu}\right)$, and second, the transport equations would acquire an additional force term of Vlasov form.

The future extensions and applications are manifold. Most important at first, I believe, is a detailed calculation for a specific situation where the concepts and formalism presented here may be illustrated and checked for consistency. For instance, it would be desirable to study a thermal (or close to thermal) parton system in this real-time description, and compare it with the well-known results in the imaginarytime formalism of finite-temperature QCD. Such a project is planned to be carried out in the near future. On the other hand, the probabilistic interpretation of the real-time evolution of a rather general multiparton system offers the opportunity to simulate the dynamical development on the basis of the master equations with Monte Carlo techniques on a computer [43].

\section{ACKNOWLEDGMENTS}

I would like to thank the following colleagues: John Ellis for his continuing support and energy; Thomas Elze for initiating this work; Berndt Müller for carefully reading a preliminary version of the manuscript and helping with numerous advice; Bo Andersson, Yuri Dokshitzer, Ulrich Heinz, Sasha Makhlin, and Pino Marchesini, for inspiring discussions and useful suggestions. Last but not least, I wish to thank the European Centre For Theoretical Studies in Nuclear Physics and Related Areas (ECT*) in Trento (Italy) for the hospitality during the International Workshop on QCD and Ultra-relativistic Heavy Ion Collisions.

\section{APPENDIX A: THE CTP FORMALISM AND THE TWO-POINT SOURCE GENERATING FUNCTIONAL}

In this appendix, I review the CTP functional formalism applied to the case of QCD. For additional reading concerning the general techniques, I refer to the extensive literature [3-5,7-9]. In the in-in formulation of quantum field theory, mentioned in Sec. II, the generating functional is defined as the in-vacuum to in-vacuum amplitude $Z[J, \hat{\rho}]$
$=\operatorname{Tr} \Sigma_{\varphi}\left\langle 0^{\text {in }} \mid \varphi\right\rangle_{J}\left\langle\varphi|\hat{\rho}| 0^{\text {in }}\right\rangle_{J}$, including possible initial state correlations represented by the density matrix $\hat{\rho}$ at $t_{0}$, and a sum over a complete set of states $\varphi$ at $t_{\infty}$. In the Heisenberg picture it is represented by

$$
Z_{P}[J, \hat{\rho}]=\operatorname{Tr}\left\{T_{P} \exp \left(i \sum_{f} \int{ }_{P} d^{4} x J^{f}(x) \Phi_{H}^{f}(x)\right) \hat{\rho}\right\},
$$

where $f=g, u, \bar{u}, d, \bar{d}, \ldots$ specifies the gluon and quark field degrees of freedom, and the $\Phi_{H}^{f}=\left(A^{\mu}, \psi, \bar{\psi}\right)$ are the corresponding Heisenberg field operators. The symbol $P$ refers to the time integration along a closed time path in the complex $t$ plane as illustrated in Fig. 2: the path goes forward from $t_{0}$ to $t_{\infty}$ on the positive branch, and then back from $t_{\infty}$ to $t_{0}$ on the negative branch. The generalized time-ordering $T_{P}$ is therefore defined such that any point on the second branch is understood at a later instant than any point on the first branch [cf. Eqs. (28) and (29)]. Utilizing Eq. (13), Eq. (A1) can be rewritten as

$$
Z_{P}\left[J_{+}, J_{-}, \hat{\rho}\right]=\operatorname{Tr}\left\{U_{J_{-}}^{\dagger}\left(t_{0}, t\right) U_{J_{+}}\left(t, t_{0}\right) \hat{\rho}\left(t_{0}\right)\right\},
$$

where $J_{+}\left(J_{-}\right)$is the source along the positive (negative) branch of Fig. 2(a). In general $J_{+} \neq J_{-}$, so that $Z_{P}$ depends on two different sources. If these are set equal, one has $Z_{P}(J, J, \rho)=\operatorname{Tr} \hat{\rho}$, which is equal to unity in the absence of initial correlations, being a statement of unitarity. The derivatives of $Z_{P}$ with respect to the sources generate the $n$-point CTP Green functions

$$
\begin{aligned}
& G_{\alpha_{1}, \ldots, \alpha_{n}}^{f_{1}, \ldots, f_{n}}\left(x_{1}, \ldots, x_{n}\right) \\
& \quad=\frac{\delta^{n} Z_{P}\left[J_{+}, J_{-}, \hat{\rho}\right]}{\delta J_{\alpha_{1}}^{f_{1}}\left(x_{1}\right) \cdots \delta J_{\alpha_{n}}^{f_{n}}\left(x_{n}\right)} \\
& \quad=(-i)^{n-1} \operatorname{Tr}\left\{T_{P} \Phi_{\alpha_{1}}^{f_{1}}\left(x_{1}\right) \cdots \Phi_{\alpha_{n}}^{f_{n}}\left(x_{n}\right) \hat{\rho}\right\},
\end{aligned}
$$

where $\alpha_{i}= \pm$, and the indices $f_{i}$ label the type of the $i$ th field operator $\Phi$ as before. The functional $Z_{P}$ can be represented as a path integral by employing the relation between the Heisenberg and interaction pictures [Eq. (16)]. One imposes boundary conditions in terms of complete sets of eigenstates of the Heisenberg fields $\Phi_{H}$ at initial time $t=t_{0}$,

$$
\begin{gathered}
\Phi_{H}\left(t_{0}, \vec{x}\right)\left|\phi^{+}\left(t_{0}\right)\right\rangle=\Phi_{I}\left(t_{0}, \vec{x}\right)\left|\phi^{+}\left(t_{0}\right)\right\rangle=\phi^{+}(\vec{x})\left|\phi^{+}\left(t_{0}\right)\right\rangle, \\
\Phi_{H}\left(t_{0}, \vec{x}\right)\left|\phi^{-}\left(t_{0}\right)\right\rangle=\phi^{-}(\vec{x})\left|\phi^{-}\left(t_{0}\right)\right\rangle,
\end{gathered}
$$

and in the remote future at $t=t_{\infty}$,

$$
\Phi_{H}\left(t_{\infty}, \vec{x}\right)\left|\varphi\left(t_{\infty}\right)\right\rangle=\varphi(\vec{x})\left|\varphi\left(t_{\infty}\right)\right\rangle .
$$

Then, making use of the completeness of the eigenstates, one obtains from Eq. (A2) the following functional integral representation for $Z_{P}$ : 


$$
\begin{aligned}
Z_{P}\left[J_{+}, J_{-}, \hat{\rho}\right]= & \int \mathcal{D} \phi^{+} \mathcal{D} \phi^{-} \mathcal{D} \varphi \\
& \times\left\langle\phi^{-}\left(t_{0}\right)\left|U_{J_{-}}^{\dagger}\left(t_{0}, t_{\infty}\right)\right| \varphi\left(t_{\infty}\right)\right\rangle \\
& \times\left\langle\varphi\left(t_{\infty}\right)\left|U_{J_{+}}\left(t_{\infty}, t_{0}\right)\right| \phi^{+}\left(t_{0}\right)\right\rangle \\
& \times\left\langle\phi^{+}\left(t_{0}\right)|\hat{\rho}| \phi^{+}\left(t_{0}\right)\right\rangle .
\end{aligned}
$$

At this point it is convenient to represent + and - by greek indices $\alpha, \beta, \gamma, \ldots$ and to introduce a $2 \times 2$ matrix $\sigma$ as a "metric tensor,"

$$
\tau_{\alpha \beta}=\tau^{\alpha \beta}=\operatorname{diag}(1,-1), \quad \alpha, \beta= \pm,
$$

and similarly higher-rank tensors,

$$
u_{\alpha \beta \gamma}=\delta_{\alpha \beta} \tau_{\beta \gamma}, \quad v_{\alpha \beta \gamma \delta}=\operatorname{sgn}(\alpha) \delta_{\alpha \beta} \delta_{\beta \gamma} \delta_{\gamma \delta},
$$

with the usual summation convention over repeated greek indices $\alpha, \beta, \ldots$. With this convention one can generalize the classical action [Eq. (4)] to account for all four field orderings on the closed time path $P$ :

$$
\begin{aligned}
I\left[\phi_{f}^{\alpha}\right] \equiv & I\left[\phi_{f}^{+}\right]-I^{*}\left[\phi_{f}^{-}\right] \\
= & \mathcal{I}^{(0)}\left[\tau_{\alpha \beta} A_{\mu}^{\alpha} A_{\nu}^{\beta}\right]+\mathcal{I}^{(0)}\left[\tau_{\alpha \beta} \bar{\psi}^{\alpha} \psi^{\beta}\right] \\
& +\mathcal{I}^{(\mathrm{int})}\left[u_{\alpha \beta \gamma} \bar{\psi}^{\alpha} A_{\mu}^{\beta} \psi^{\gamma}, u_{\alpha \beta \gamma}\left(\partial_{\mu} A_{\nu}^{\alpha}\right) A_{\mu}^{\beta} A_{\nu}^{\gamma},\right. \\
& \left.v_{\alpha \beta \gamma \delta} A_{\mu}^{\alpha} A_{\nu}^{\beta} A_{\mu}^{\gamma} A_{\nu}^{\delta}\right],
\end{aligned}
$$

where the correspondence of the terms $\mathcal{I}$ with the ones of Eq. (4) is obvious (the color indices are suppressed here). Also, the following shorthand notation for the integration over the space-time variables will be used in the functional sense:

$$
\begin{gathered}
J \phi \equiv \int_{P} d^{4} x J(x) \phi(x), \\
\phi K \phi \equiv \int_{P} d^{4} x d^{4} y \phi(x) K(x, y) \phi(y) .
\end{gathered}
$$

Returning to the functional integral [Eq. (A6)], I now utilize the above conventions and exploit the fact that the first two amplitudes are just the ordinary transition matrix elements in the presence of $J_{+}$and $J_{-}$, whereas the density matrix element incorporates the initial state correlations at $t_{0}$ at the end points of the closed time path $P$. I obtain the path integral representation for $Z_{P}$ in analogy with the usual field theory $[11,12]$

$$
\begin{aligned}
Z_{P}\left[J_{+}, J_{-}, \hat{\rho}\right]= & \int \prod_{f} \mathcal{D} \phi_{f}^{\alpha} \exp \left[i \left(I\left[\phi_{f}^{\alpha}\right]\right.\right. \\
& \left.\left.+\sum_{f} J_{f, \alpha} \phi_{f}^{\alpha}\right)\right] \mathcal{N}[\hat{\rho}] .
\end{aligned}
$$

Here $\mathcal{D} \phi_{f}^{\alpha} \equiv \mathcal{D} \phi_{f}^{+} \mathcal{D} \phi_{f}^{-}$, and I suppressed the formal presence of the Faddeev-Popov determinant associated with the gauge freedom, because for the class of ghost-free gauges [Eq. (3)] it is equal to unity. The functional $\mathcal{N}[\hat{\rho}]$ is the density matrix element containing the initial state correlations that may be represented by a nonlocal source functional $K$ as

$$
\mathcal{N}[\hat{\rho}]=\left\langle\phi_{+}\left(t_{0}\right)|\hat{\rho}| \phi_{-}\left(t_{0}\right)\right\rangle \equiv \exp \left(i \sum_{f} K_{f}\left[\phi_{+}, \phi_{-}\right]\right)
$$

When expanded functionally as

$$
\begin{aligned}
K_{f}\left[\phi_{+}, \phi_{-}\right]= & K^{f}+K_{\alpha}^{f} \phi^{\alpha}+\frac{1}{2} \sum_{f^{\prime}} K_{\alpha \beta}^{f f^{\prime}} \phi_{f}^{\alpha} \phi_{f^{\prime}}^{\beta} \\
& +\frac{1}{6} \sum_{f^{\prime} f^{\prime \prime}} K_{\alpha \beta \gamma}^{f f^{\prime} f^{\prime \prime}} \phi_{f}^{\alpha} \phi_{f^{\prime}}^{\beta} \phi_{f^{\prime \prime}}^{\gamma}+\cdots,
\end{aligned}
$$

Eq. (A11) becomes a functional of an infinite number of nonlocal sources [8], which, however, contribute only at $t=t_{0}$, corresponding to the initial state correlations,

$$
\begin{aligned}
Z_{P}\left[J_{+}, J_{-}, \hat{\rho}\right] \equiv & Z_{P}\left[J_{\alpha}, K_{\alpha \beta}, \ldots\right] \\
= & \int \prod_{f, \alpha} \mathcal{D} \phi_{f}^{\alpha} \exp \left\{i \left[I\left[\phi_{f}\right]+\sum_{f}\left(J_{\alpha}^{f} \phi_{f}^{\alpha}\right.\right.\right. \\
& \left.\left.\left.+\frac{1}{2} \sum_{f^{\prime}} \phi_{f}^{\alpha} K_{\alpha \beta}^{f f^{\prime}} \phi_{f^{\prime}}^{\beta}+\ldots\right)\right]\right\}, \quad(\mathrm{A} 14)
\end{aligned}
$$

where the constant term $K^{f}$ has been absorbed into the normalization and the local initial state kernel $K_{\alpha}^{f}(x)$ has been combined with the external source term $J_{\alpha}^{f}(x)$, i.e., $J_{\alpha}^{f} \equiv J_{\alpha}^{f}+K_{\alpha}^{f}$.

The corresponding generating functional for the connected Green functions $W_{P}$ is defined as usual by the logarithm of $Z_{P}$ :

$$
W_{P}\left[J^{\alpha}, K^{\alpha \beta}, K^{\alpha \beta \gamma}, \ldots\right]=-i \ln Z_{P}\left[J^{\alpha}, K^{\alpha \beta}, K^{\alpha \beta \gamma}, \ldots\right] .
$$

The functional derivatives of $W_{P}$ with respect to the local sources $J_{f}^{\alpha}(x)$ define gluon and quark mean fields $\widetilde{\phi}_{f}^{\alpha}(x)$ as the expectation values of the single field operators, which either can arise through nonvanishing external sources, or, in the case of gluons, may be generated dynamically by the system itself depending on the initial conditions. Similarly, the variation of $W_{P}$ with respect to the nonlocal kernels $K_{f f^{\prime}}^{\alpha \beta}\left(x, x^{\prime}\right), K_{f f^{\prime} f^{\prime \prime}}^{\alpha \beta \gamma}\left(x, x^{\prime}, x^{\prime \prime}\right), \ldots$, gives the the $n$-point Green functions $G_{f f^{\prime}}^{\alpha \beta}\left(x, x^{\prime}\right), G_{f f^{\prime} f^{\prime \prime}}^{\alpha \beta \gamma}\left(x, x^{\prime}, x^{\prime \prime}\right)$, etc., for the different particle species, which are the expectation values of products of $n$ field operators. On the basis of Eqs. (A14) and (A15), the explicit definitions [11] for the mean fields and the Green functions are given by the functional derivatives of $W_{P}$ with respect to the sources $J$ and $K$ :

$$
\frac{\delta W_{P}}{\delta J_{\alpha}^{f}}=\widetilde{\phi}_{f}^{\alpha}
$$

$$
\frac{\delta W_{P}}{\delta K_{\alpha \beta}^{f f^{\prime}}}=\frac{1}{2}\left(i G_{f f^{\prime}}^{\alpha \beta}+\widetilde{\phi}_{f}^{\alpha} \widetilde{\phi}_{f^{\prime}}^{\beta}\right),
$$




$$
\frac{\delta W_{P}}{\delta K_{\alpha \beta \gamma}^{f f^{\prime} f^{\prime \prime}}}=\frac{1}{6}\left(G_{f f^{\prime} f^{\prime \prime}}^{\alpha \beta}+3 i G_{f f^{\prime}}^{\alpha \beta} \widetilde{\phi}_{f^{\prime \prime}}^{\gamma}+\widetilde{\phi}_{f}^{\alpha} \widetilde{\phi}_{f^{\prime}}^{\beta} \widetilde{\phi}_{f^{\prime \prime}}^{\gamma}\right) \text {, etc. }
$$

Note that with these definitions, the Green functions $G_{f f^{\prime} \ldots}^{\alpha \beta \cdots}$ describe the actual net change of $W_{P}$, i.e., $\delta W_{P} / \delta K_{\alpha \beta}^{f f^{\prime} \cdots}$ with the "trivial', product $\widetilde{\phi}_{f}^{\alpha} \widetilde{\phi}_{f^{\prime}}^{\beta} \ldots$ subtracted.

One immediately recognizes the infinite hierarchy of the Green functions, the complete knowledge of which would correspond to the full solution of the theory. Clearly, in practice one must truncate this infinite series. In what follows, I will assume that all $n$-point sources of order $n \geqslant 3$ (i.e., the kernels $K^{\alpha \beta \gamma}$, etc.) are negligible and thus can be omitted. Such an approximation is justified if the higher-order correlations generated by the $n \geqslant 3$ source terms are comparably small and the quantum dynamics of the system can be sufficiently well described by single-particle distributions corresponding to the two-point functions. In fact, this is the very hypothesis of the parton description of QCD at large energies, where higher-order correlations ("higher-twist" effects) are kinematically suppressed by powers of a large momentum scale $Q^{-2}$ corresponding to an approximate factorization of dominant short-distance two-point correlations and larger-distance three-, four-, . . , point correlations associated with multiparticle effects, an approximation which becomes exact in the asymptotic limit [27]. In this approximation the generating functional [Eq. (A14)] then explicitly reads

$$
\begin{aligned}
Z_{P}\left[J^{\mu}, j, \bar{j}, K^{\mu \nu}, k\right]= & e^{i W_{P}\left[J^{\mu}, j, \bar{j} K^{\mu \nu}, k\right]} \\
= & \int \mathcal{D} A_{\alpha}^{\mu} \mathcal{D} \psi_{\alpha} \mathcal{D} \bar{\psi}_{\alpha} \exp \left[i \left(I\left[A_{\alpha}^{\mu}, \psi_{\alpha}, \bar{\psi}_{\alpha}\right]\right.\right. \\
& +J_{\mu}^{\alpha} A_{\alpha}^{\mu}+j^{\alpha} \bar{\psi}_{\alpha}+\frac{1}{2} A_{\alpha}^{\mu} K_{\mu \nu}^{\alpha \beta} A_{\beta}^{\nu} \\
& +\bar{\psi}_{\left.\left.\alpha k^{\alpha \beta} \psi_{\beta}\right)\right]}
\end{aligned}
$$

which is the expression I stated in Eq. (25) of Sec. II B. Since the present interest concerns only cases where no external sources are present, one obtains from Eq. (A17) the mean fields and the two-point functions for gluons and quarks by taking into account the fact that in the absence of external sources the establishment of a local colored mean field can only occur for the gluons, but not for quarks or antiquarks. Because of their bosonic character the production of gluons can lead to a dynamically generated coherent field acting as a background medium, whereas the production of quarks and antiquarks occurs only in pairs and cannot yield a coherent mean field. Furthermore, a physical gluon mean field is determined by the equality $\widetilde{A_{+}^{\mu}}=\widetilde{A_{-}^{\mu}} \equiv \widetilde{A^{\mu}}$. Hence one gets, from Eq. (A17), using Eq. (A16),

$$
\frac{\delta W_{P}}{\delta J_{\alpha}^{\mu}(x)}=\widetilde{A_{\mu}}(x), \quad \frac{\delta W_{P}}{\delta \overline{j_{\alpha}}(x)}=\frac{\delta W_{P}}{\delta j_{\alpha}(x)}=0,
$$

$$
\begin{gathered}
\frac{\delta W_{P}}{\delta K_{\alpha \beta}^{\mu \nu}(x, y)}=\frac{1}{2}\left[i D_{\mu \nu}^{\alpha \beta}(x, y)+\widetilde{A}_{\mu}^{\alpha}(x) \widetilde{A}_{\nu}^{\beta}(y)\right], \\
\frac{\delta W_{P}}{\delta k_{\alpha \beta}(x, y)}=-i S^{\alpha \beta}(x, y),
\end{gathered}
$$

where, as a reminder $\alpha, \beta= \pm$, not to be confused with color indices, which I suppressed in this appendix.

\section{APPENDIX B: FROM QUANTUM FIELD DESCRIPTION TO KINETIC THEORY}

In this appendix I explain steps that lead from the DysonSchwinger equations (44) and (45) to the kinetic counterparts, the renormalization equations (67) and transport equations (68). A good separation between the quantum scale of short-distance fluctuations and the statistical-kinetic scale is the essential requirement for recasting the quantumtheoretical problem, formulated in terms of the two-point Green functions $G(x, y)$, into the much simpler form of kinetic theory, employing Wigner transforms $G(r, p)$. In the picture of cellular space-time, constructed in Sec. III A, a clearly defined separation between the two scales is controlled by the characteristic size of the space-time cells with volume $\Omega \simeq \mu^{-4}$, by choosing $\mu$ such that $\Delta r_{\text {qua }}$ $\leqslant \mu^{-1}<\Delta r_{\text {kin }}$, i.e., the cell size is larger than the range of short-distance quantum fluctuations, $\Delta r_{\text {qua }} \leqslant \mu^{-1}$, but small compared to the mean free path of the quanta between their kinetic, statistical interactions, $\Delta r_{\text {kin }}$. This is illustrated in Figs. 6 and 7. Therefore the correlation between different cells is negligible by design, and the only relevant case is when the points $x$ and $y$ in the argument of $G(x, y)$ lie in the same cell. In the interior of each cell, one can then assume approximate translation invariance, because large-distance inhomogeneities of space-time are, by construction, not resolvable within the small cell volume. Thus, for each individual cell, one can Fourier-transform the Green functions over the cell volume, and use the common machinery of propagator theory as for homogeneous systems, or the vacuum. Specifically, introducing the variables

$$
r=\frac{1}{2}(x+y), \quad s=x-y,
$$

one transforms the Green functions $G \equiv D_{\mu \nu}, S$ with respect to the "relative coordinate", $s$, whereas the "absolute coordinate" $r$ serves as a cell label:

$$
\begin{aligned}
G(x, y) & =\int \frac{d^{4} p}{(2 \pi)^{4}} e^{-i p \cdot(x-y)} G\left(\frac{x+y}{2}, x-y\right) \\
& =\int \frac{d^{4} p}{(2 \pi)^{4}} e^{-i p \cdot s} G(r, s),
\end{aligned}
$$

where $G(r, p)$ is called the Wigner transform of $G(x, y)$, and similarly for the self-energies $\mathcal{E}(x, y) \equiv \Pi_{\mu \nu}, \Sigma$,

$$
\begin{aligned}
\mathcal{E}(x, y) & =\int \frac{d^{4} q}{(2 \pi)^{4}} e^{-i q \cdot(x-y)} \mathcal{E}\left(\frac{x+y}{2}, x-y\right) \\
& =\int \frac{d^{4} q}{(2 \pi)^{4}} e^{-i q \cdot s} \mathcal{E}(r, s) .
\end{aligned}
$$


If the separation between quantum and kinetic scales were perfect (as in vacuum, where $\Delta r_{\text {kin }}=\infty$ ), then the $r$ dependence would drop out and the Wigner transforms reduce to the ordinary Fourier transforms $G(p)$ and $\mathcal{E}(q)$. In the case of moderately inhomogeneous media, meaning that the Green functions and self-energies vary only slowly with $r$ and are strongly peaked around $s=x-y$, as I consider here, then one can expand the Green functions $G(x, y)=W\left(r+\frac{1}{2} s, r-\frac{1}{2} s\right)=W(r, s)$ in a series of gradients,

$$
W(r+s, s) \simeq W(r, s)+s \cdot \partial_{r} W(r, s)+\cdots,
$$

and analogously for the self-energies $\mathcal{E}$. The great advantage of the Wigner transformation and the gradient expansion is that, on the one hand, the fundamental Dyson-Schwinger equations (44) and (45) do not change their formal structure when Wigner transformed; on the other hand, however, within the kinetic approximation based on the gradient expansion [Eq. (152)], they become algebraic differential equations rather than integro-differential equations like the original ones. For example, when rewriting the integral terms on the right side of Eqs. (44) and (45) by substituting the Wigner transforms for the Green functions $G=D^{\mu \nu}, S$, Eq. (B2) and self-energies $\mathcal{E}=\Pi^{\mu \nu}, \Sigma$, Eq. (B3), and exploiting the expansion [Eq. (B4)] up to first order, one finds for the terms $\int \mathcal{E} G \equiv \int \Pi_{\mu \sigma} D_{\nu}^{\sigma}$ or $\int \mathcal{E} G \equiv \int \Sigma S$ :

$$
\begin{aligned}
\int d^{4} x^{\prime} \mathcal{E}\left(x, x^{\prime}\right) G\left(x^{\prime}, y\right)= & \int \frac{d^{4} q}{(2 \pi)^{4}} \frac{d^{4} p}{(2 \pi)^{4}} \int d^{4} x^{\prime} \\
& \times e^{-i q \cdot\left(x-x^{\prime}\right)} \mathcal{E}\left(\frac{x+x^{\prime}}{2}, x-x^{\prime}\right) \\
& \times e^{-i p \cdot\left(x^{\prime}-y\right)} G\left(\frac{x^{\prime}+y}{2}, x^{\prime}-y\right) .
\end{aligned}
$$

The integrand will be significantly different from zero only if $x^{\prime}$ lies within the same cell as $x$ and $y$, in which case $\frac{1}{2}\left(x+x^{\prime}\right) \simeq \frac{1}{2}\left(x^{\prime}+y\right) \simeq r$. Therefore Eq. (B5) reduces to

$$
\begin{aligned}
& \int d^{4} x^{\prime} \mathcal{E}\left(x, x^{\prime}\right) G\left(x^{\prime}, y\right) \\
& =\int \frac{d^{4} p}{(2 \pi)^{4}}\left\{e^{-i p \cdot(x-y)} \mathcal{E}(r, p) G(r, p)+\Delta(r, p)\right\},
\end{aligned}
$$

where $\Delta(r, p)$ embodies the corrections to the ideal separation of cells. In terms of the gradient expansion [Eq. (B4)], the first order correction which is $O(\hbar)$ is given by

$$
\begin{aligned}
\Delta(r, p) & =\frac{i}{2} \frac{\partial \mathcal{E}(r, p)}{\partial p_{\mu}} \frac{\partial G(r, p)}{\partial r^{\mu}}-\frac{i}{2} \frac{\partial \mathcal{E}(r, p)}{\partial r^{\mu}} \frac{\partial G(r, p)}{\partial p^{\mu}} \\
& \equiv \frac{i}{2}\left[\left(\partial_{p} \mathcal{E}\right) \cdot\left(\partial_{r} G\right)-\left(\partial_{r} \mathcal{E}\right) \cdot\left(\partial_{p} G\right)\right] .
\end{aligned}
$$

In general, the convolution between two functions $f$ and $g$ is given by

$$
\begin{aligned}
\int d^{4} x^{\prime} f\left(x, x^{\prime}\right) g\left(x^{\prime}, y\right) \rightarrow & \exp \left[\frac{i}{2}\left(\partial_{p}^{(f)} \cdot \partial_{r}^{(g)}-\partial_{r}^{(f)} \cdot \partial_{p}^{(g)}\right)\right] \\
& \times f(r, p) g(r, p) .
\end{aligned}
$$

The gradient expansion [Eq. (B4)] corresponds to keeping only the first two terms in the Taylor series of the exponential function, which gives the following set of conversion rules:

$$
\begin{gathered}
\int d^{4} x^{\prime} f\left(x, x^{\prime}\right) g\left(x^{\prime}, y\right) \\
\rightarrow f(r, p) g(r, p)+\frac{i}{2}\left[\left(\partial_{p} f\right) \cdot\left(\partial_{r} g\right)-\left(\partial_{r} f\right) \cdot\left(\partial_{p} g\right)\right], \\
h(x) g(x, y) \rightarrow h(r) g(r, p)-\frac{i}{2}\left(\partial_{r} h\right) \cdot\left(\partial_{p} g\right), \\
h(y) g(x, y) \rightarrow h(r) g(r, p)+\frac{i}{2}\left(\partial_{r} h\right) \cdot\left(\partial_{p} g\right), \\
\partial_{x}^{\mu} f(x, y) \rightarrow\left(-i p^{\mu}+\frac{1}{2} \partial_{r}^{\mu}\right) f(r, p), \\
\partial_{y}^{\mu} f(x, y) \rightarrow\left(+i p^{\mu}+\frac{1}{2} \partial_{r}^{\mu}\right) f(r, p) .
\end{gathered}
$$

If one applies these rules now to the Dyson-Schwinger equations (44) and (45), which upon setting for simplicity the mean-field contributions $\widetilde{\mu}_{g}=\tilde{\mu}_{q}=0$, read

$$
\begin{aligned}
\vec{\square}_{x, \mu \rho} D_{a b}^{\rho \nu}(x, y)= & \delta_{a b} g^{\mu \nu} \delta_{P}^{4}(x, y) \\
& -\int_{P} d^{4} x^{\prime} \Pi_{\sigma, a, b^{\prime}}^{\mu}\left(x, x^{\prime}\right) D_{b^{\prime} b}^{\sigma \nu}\left(x^{\prime}, y\right), \\
D_{a b}^{\rho \nu}(x, y) \overleftarrow{\square}_{y, \mu \rho}= & \delta_{a b} g_{\mu \nu} \delta_{P}^{4}(x, y) \\
& -\int_{P} d^{4} x^{\prime} D_{\sigma, a, b^{\prime}}^{\mu}\left(x, x^{\prime}\right) \Pi_{b^{\prime} b}^{\sigma \nu}\left(x^{\prime}, y\right),
\end{aligned}
$$

and

$$
\begin{gathered}
i \gamma \cdot \vec{\partial}_{x} S_{i j}(x, y)=\delta_{i j} \delta_{P}^{4}(x, y)+\int_{P} d^{4} x^{\prime} \Sigma_{i k}\left(x, x^{\prime}\right) S_{k j}\left(x^{\prime}, y\right), \\
-S_{i j}(x, y) i \gamma \cdot \grave{\partial}_{y}=\delta_{i j} \delta_{P}^{4}(x, y)+\int_{P} d^{4} x^{\prime} S_{i k}\left(x, x^{\prime}\right) \Sigma_{k j}\left(x^{\prime}, y\right),
\end{gathered}
$$

one finds on the basis of the gradient expansion [Eq. (B4)] a set of corresponding matrix equations for the Wigner transforms of the gluon and quark Green functions 


$$
\begin{aligned}
\left(-k^{2}+\right. & \left.\frac{1}{4} \vec{\partial}_{r}^{2}-i k \cdot \vec{\partial}_{r}\right) D_{a b}^{\mu \nu}(r, k) \\
= & d^{\mu \nu}(k) \delta_{a b} \hat{1}_{P}-(\Pi D)_{a b}^{\mu \nu}-\frac{i}{2}\left[\left(\partial_{k} \Pi\right) \cdot\left(\partial_{r} D\right)\right. \\
& \left.-\left(\partial_{r} \Pi^{\mu \sigma}\right) \cdot\left(\partial_{k} D_{\sigma}^{\nu}\right)\right]_{a b}^{\mu \nu}, \\
D_{a b}^{\mu \nu}(r, k)\left(-k^{2}+\frac{1}{4} \overleftarrow{\partial}_{r}^{2}+i k \cdot \grave{\partial}_{r}\right) & \\
= & d^{\mu \nu}(k) \delta_{a b} \hat{1}_{P}-(D \Pi)_{a b}-\frac{i}{2}\left[\left(\partial_{k} D\right) \cdot\left(\partial_{r} \Pi\right)\right. \\
& \left.-\left(\partial_{r} D\right) \cdot\left(\partial_{k} \Pi\right)\right]_{a b}^{\mu \nu},
\end{aligned}
$$

and

$$
\begin{aligned}
{\left[\gamma \cdot\left(p+\frac{i}{2} \vec{\partial}_{r}\right)\right] S_{i j}(r, p)=} & \delta_{i j} \hat{1}_{P}+(\Sigma S)_{i j}+\frac{i}{2}\left[\left(\partial_{p} \Sigma\right) \cdot\left(\partial_{r} S\right)\right. \\
& \left.-\left(\partial_{r} \Sigma\right) \cdot\left(\partial_{p} S\right)\right]_{i j}, \\
S_{i j}(r, p)\left[\gamma \cdot\left(p-\frac{i}{2} \overleftarrow{\partial}_{r}\right)\right]= & \delta_{i j} \hat{1}_{P}+(S \Sigma)_{i j}+\frac{i}{2}\left[\left(\partial_{p} S\right) \cdot\left(\partial_{r} \Sigma\right)\right. \\
& \left.-\left(\partial_{r} S\right) \cdot\left(\partial_{p} \Sigma\right)\right]_{i j},
\end{aligned}
$$

where

$$
\hat{1}_{P}= \begin{cases}\hat{1} & \text { for } F, \bar{F}, \\ 0 & \text { for }>,<,\end{cases}
$$

recalling that $G \equiv D^{\mu \nu}, S$ and the self-energies $\mathcal{E} \equiv \Pi^{\mu \nu}, \Sigma$ each represent a $2 \times 2$ matrix as defined by Eq. (27),

$$
G=\left(\begin{array}{cc}
G^{F} & G^{>} \\
G^{<} & G^{\bar{F}}
\end{array}\right), \quad \mathcal{E}=\left(\begin{array}{ll}
\mathcal{E}^{F} & \mathcal{E}^{>} \\
\mathcal{E}^{<} & \mathcal{E}^{\bar{F}}
\end{array}\right) .
$$

Adding the two equations of (B12) [(B13)] yields the imaginary parts as the passage to the renormalization equations stated in Eq. (67), whereas subtracting the two equations of (B12) [(B13)] gives the real parts as the transport equations (68). For the gluon Wigner functions one obtains

$$
\begin{aligned}
\left(k^{2}-\frac{1}{4} \partial_{r}^{2}\right) D_{a b}^{\mu \nu}(r, k)= & -d^{\mu \nu}(k) \delta_{a b} \hat{1}_{P}+\frac{1}{2}\left(\{\Pi, D\}_{+}\right)_{a b}^{\mu \nu} \\
& +\frac{i}{4} \mathcal{G}_{a b}^{\mu \nu(-)}, \\
k \cdot \partial_{r} D_{a b}^{\mu \nu}(r, k)=- & \frac{i}{2}\left([\Pi, D]_{-}\right)_{a b}^{\mu \nu}+\frac{1}{4} \mathcal{G}_{a b}^{\mu \nu(+)},
\end{aligned}
$$

where $[A, B]_{-} \equiv A B-B A,\{A, B\}_{+} \equiv A B+B A$, and

$$
\begin{aligned}
& \mathcal{G}^{\mu \nu(-)}=\left[\partial_{k}^{\lambda} \Pi_{\sigma}^{\mu}, \partial_{\lambda}^{r} D^{\sigma \nu}\right]_{-}-\left[\partial_{r}^{\lambda} \Pi_{\sigma}^{\mu}, \partial_{\lambda}^{k} D^{\sigma \nu}\right]_{-}, \\
& \mathcal{G}^{\mu \nu(+)}=\left\{\partial_{k}^{\lambda} \Pi_{\sigma}^{\mu}, \partial_{\lambda}^{r} D^{\sigma \nu}\right\}_{+}-\left\{\partial_{r}^{\lambda} \Pi_{\sigma}^{\mu}, \partial_{\lambda}^{k} D^{\sigma \nu}\right\}_{+} .
\end{aligned}
$$

For the quark Wigner functions the corresponding equations read

$$
\begin{aligned}
\frac{1}{2}\left\{\gamma \cdot p, S_{i j}(r, p)\right\}_{+}= & \delta_{i j} \hat{1}_{P}-\frac{i}{2}\left(\left[\gamma \cdot \partial_{r}, S\right]_{-}\right)_{i j} \\
& +\frac{1}{2}\left(\{\Sigma, S\}_{+}\right)_{i j}+\frac{i}{4} \mathcal{F}_{i j}^{(-)}, \\
\frac{1}{2}\left\{\gamma \cdot \partial_{r}, S_{i j}(r, p)\right\}_{+}= & \frac{i}{2}\left([\gamma \cdot p, S]_{-}\right)_{i j}-\frac{i}{2}\left([\Sigma, S]_{-}\right)_{i j} \\
& +\frac{1}{4} \mathcal{F}_{i j}^{(+)},
\end{aligned}
$$

where

$$
\begin{aligned}
& \mathcal{F}^{(-)}=\left[\partial_{p}^{\lambda} \Sigma, \partial_{\lambda}^{r} S\right]_{-}-\left[\partial_{r}^{\lambda} \Sigma, \partial_{\lambda}^{p} S\right]_{-}, \\
& \mathcal{F}^{(+)}=\left\{\partial_{p}^{\lambda} \Sigma, \partial_{\lambda}^{r} S\right\}_{+}-\left\{\partial_{r}^{\lambda} \Sigma, \partial_{\lambda}^{p} S\right\}_{+} .
\end{aligned}
$$

The equations (B18) for quark propagators can be formally brought in the same form as Eq. (B16) for the gluon propagators by multiplying the first equation of (B13) by $\gamma \cdot\left(p+i / 2 \overleftarrow{\partial}_{r}\right) \delta_{l i}+\Sigma_{l i}$ from the left, and the second equation of (B13) by $\gamma \cdot\left(p-i / 2 \overleftarrow{\partial}_{r}\right) \delta_{j l}+\Sigma_{j l}$ from the right, and then adding and subtracting the resulting equations:

$$
\begin{gathered}
\left(p^{2}-\frac{1}{4} \partial_{r}^{2}\right) S_{i j}(r, p)=(\gamma \cdot p+\Sigma) \delta_{i j} \hat{1}_{P}+\frac{1}{2}\left(\left\{\Sigma^{2}, S\right\}_{+}\right)_{i j} \\
+\frac{i}{4} \mathcal{A}_{i j}^{(+)}-\frac{1}{8} \mathcal{B}_{i j}^{(-)}, \\
p \cdot \partial_{r} S_{i j}(r, p)=\frac{1}{2}\left(\gamma \cdot \partial_{r}\right) \delta_{i j} \hat{1}_{P}-\frac{i}{2}\left(\left[\Sigma^{2}, S\right]_{-}\right)_{i j}+\frac{1}{4} \mathcal{A}_{i j}^{(-)} \\
+\frac{i}{8} \mathcal{B}_{i j}^{(+)},
\end{gathered}
$$

where the notation $\Sigma_{i j}=\delta_{i j} \Sigma$ is employed, and

$$
\begin{gathered}
\mathcal{A}^{( \pm)}=\frac{1}{2}\left[(\gamma \cdot p+\widetilde{\Sigma})\left(\mathcal{F}^{(-)}+\mathcal{F}^{(+)}\right)\right. \\
\left. \pm\left(\mathcal{F}^{(-)}-\mathcal{F}^{(+)}\right)(\gamma \cdot p+\widetilde{\Sigma})\right], \\
\mathcal{B}^{( \pm)}=\frac{1}{2}\left[\left(\gamma \cdot \vec{\partial}_{r}\right)\left(\mathcal{F}^{(-)}+\mathcal{F}^{(+)}\right) \pm\left(\mathcal{F}^{(-)}-\mathcal{F}^{(+)}\right)\left(\gamma \cdot \stackrel{\leftarrow}{\partial}_{r}\right)\right] .
\end{gathered}
$$

Due to the $2 \times 2$ matrix character of Eqs. (B16), (B18), and (B20), the four components $F, \bar{F},>,<$ of the Green functions $D^{\mu \nu}$ and $S$ and self-energies $\Pi^{\mu \nu}$ and $\Sigma$ mix, so that each of these equations actually represents a nontrivial coupled set of four equations. However, as noted in Sec. III C, in the physical representation [Eq. (77)],

$$
\breve{G}=\left(\begin{array}{cc}
0 & G^{A} \\
G^{R} & G^{C}
\end{array}\right), \quad \breve{\mathcal{E}}=\left(\begin{array}{cc}
\mathcal{E}^{C} & \mathcal{E}^{R} \\
\mathcal{E}^{A} & 0
\end{array}\right),
$$

one has the great advantage that the retarded and advanced functions $G^{R(A)}$ are determined exclusively by the $R$ and $A$ components, and only the equation for $G^{C}$ involves a mixing 
of these. Omitting for lucidity the gradient terms $\mathcal{G}$ and $\mathcal{F}$, Eqs. (B16) become in the physical representation a selfcontained set for the retarded and advanced functions:

$$
\begin{gathered}
\left(k^{2}-\frac{1}{4} \partial_{r}^{2}\right) D_{a b}^{\mu \nu R(A)}(r, k) \\
=-d^{\mu \nu}(k) \delta_{a b}+\frac{1}{2}\left[\Pi^{R(A)} D^{R(A)}+D^{R(A)} \Pi^{R(A)}\right]_{a b}^{\mu \nu}, \\
k \cdot \partial_{r} D_{a b}^{\mu \nu R(A)}(r, k)=-\frac{i}{2}\left[\Pi^{R(A)} D^{R(A)}-D^{R(A)} \Pi^{R(A)}\right]_{a b}^{\mu \nu},
\end{gathered}
$$

and

$$
\begin{aligned}
\frac{1}{2}\left\{\gamma \cdot p, S_{i j}^{R(A)}(r, p)\right\}_{+}= & \delta_{i j}-\frac{i}{4}\left(\left[\gamma \cdot \partial_{r}, S^{R(A)}\right]_{-}\right)_{i j} \\
& +\frac{1}{2}\left(\left\{\Sigma^{R(A)}, S^{R(A)}\right\}_{+}\right)_{i j}, \\
\frac{1}{2}\left\{\gamma \cdot \partial_{r}, S_{i j}^{R(A)}(r, p)\right\}_{+}= & \frac{i}{2}\left(\left[\gamma \cdot p, S^{R(A)}\right]_{-}\right)_{i j} \\
& -\frac{i}{2}\left(\left[\Sigma^{R(A)}, S^{R(A)}\right]_{-}\right)_{i j},
\end{aligned}
$$

plus a set of mixed equations for the correlation functions,

$$
\begin{aligned}
& \left(k^{2}-\frac{1}{4} \partial_{r}^{2}\right) D_{a b}^{\mu \nu \gtrless}(r, k) \\
& =-\frac{1}{2}\left(\Pi^{\gtrless} D^{A}+\Pi^{R} D^{\gtrless}+D^{\gtrless} \Pi^{A}+D^{R} \Pi^{\gtrless}\right)_{a b}^{\mu \nu}, \\
& k \cdot \partial_{r} D_{a b}^{\mu \nu \gtrless}(r, k) \\
& =-\frac{i}{2}\left(\Pi^{\gtrless} D^{A}+\Pi^{R} D^{\gtrless}-D^{\gtrless} \Pi^{A}-D^{R} \Pi^{\gtrless}\right)_{a b}^{\mu \nu},
\end{aligned}
$$

with $D_{\mu \nu}^{C}=D_{\mu \nu}^{>}+D_{\mu \nu}^{<}$, and

$$
\begin{aligned}
\left\{\gamma \cdot p, S_{i j}^{\gtrless}(r, p)\right\}_{+}= & -\frac{i}{2}\left(\left[\gamma \cdot \partial_{r}, S^{\gtrless}\right]_{-}\right)_{i j}+\left(\Sigma^{\gtrless} S^{A}+\Sigma^{R} S^{\gtrless}\right. \\
+ & \left.S^{\gtrless} \Sigma^{A}+S^{R} \Sigma^{\gtrless}\right)_{i j}, \\
i\left\{\gamma \cdot \partial_{r}, S_{i j}^{\gtrless}(r, p)\right\}_{+}= & -\left(\left[\gamma \cdot p, S^{\gtrless}\right]_{-}\right)_{i j}+\left(\Sigma^{\gtrless} S^{A}+\Sigma^{R} S^{\gtrless}\right. \\
& \left.-S^{\gtrless} \Sigma^{A}-S^{R} \Sigma^{\gtrless}\right)_{i j},
\end{aligned}
$$

with $S^{C}=S^{>}+S^{<}$.

\section{APPENDIX C: THE RENORMALIZATION FUNCTIONS AND THE SPECTRAL DENSITIES OF PARTONS}

In the following I explain in more detail the steps that lead from the determining equation for the retarded selfenergies $\hat{\Pi}$ and $\hat{\Sigma}$, Eqs. (103) and (104), to the solution for the renormalization functions $\Delta_{g}$ and $\Delta_{q}$, Eqs. (109), and finally to the evolution equations for the phase-space densities $F_{g}$ and $F_{q}$, Eq. (115). I exemplify the procedure for the simpler case of the quark self-energy. The case of the gluon self-energy is more elaborate, but conceptually it is completely analogous. I confine myself here to the leading log approximation (LLA), referring to Refs. [27,28] for additional reading.

The quantity of interest is hence the quark self-energy $\Sigma_{i j}=\delta_{i j} p^{2} \hat{\Sigma}$, Eq. (90), given by Eq. (99). As explained in Sec. II A, when studying short-distance dynamics around the light cone, it is appropriate and most convenient to work in the planar axial gauge $n \cdot A=0$, Eqs. (2) and (3), with the constant vector $n^{\mu}$ satisfying $n^{2} \ll 1$. Parametrizing it as $n^{\mu}=(a+b, 0,0, a-b)$ then requires $n^{2}=4 a b \ll 1$. Without loss of generality, one may set $b=1$ and $a \ll 1 / 4$, so that the scalar product of $n$ with some four-vector $q$ is $n \cdot q=q^{+}+a q^{-} \simeq q^{+} \quad$ with $\quad q^{ \pm}=q_{0} \pm q_{3} \quad$ and $\quad q^{+} q^{-}$ $=q^{2}-q_{\perp}^{2} \simeq q^{2} \ll q^{+2}$. Let me then proceed with Eq. (104) for the variation of the scalar quark self-energy function $\hat{\Sigma}(r, p)$ of a quark with momentum $p$ and virtuality $p^{2} \ll p^{+2}$, within a given space-time cell of volume $\Omega(r)=\Delta r^{0} \Delta^{3} r=\mu^{-4}(r)$ around $r$ (cf. Sec. III A):

$$
\begin{aligned}
p^{2} \frac{\partial}{\partial p^{2}} & \hat{\Sigma}^{R(A)}(r, p) \\
= & -g_{s}^{2} C_{F}(2 \pi i) \int \frac{d^{4} p^{\prime}}{(2 \pi)^{4} i} \int_{r_{0}-1 /(2 \mu)}^{r_{0}+1 /(2 \mu)} d \tau \mathcal{T}\left(p^{\prime} \tau\right) \\
& \times \frac{\partial \Delta_{q}\left(r, p^{\prime}\right)}{\partial p^{\prime 2}} \frac{\partial}{\partial k^{\prime \prime 2}} \\
& \times\left[V_{q q g}^{2}\left(r ; p^{2}, p^{\prime 2}, k^{\prime \prime 2}, \kappa_{p}, \kappa_{p^{\prime}}, \kappa_{k^{\prime \prime}}\right)\right. \\
& \left.\times \Delta_{g}\left(r, k^{\prime \prime}\right)\right] \times \mathcal{U}_{q}^{q g}\left(p^{\prime}, k^{\prime \prime}, n\right)
\end{aligned}
$$

with

$$
\kappa_{p}=\frac{(n \cdot p)^{2}}{n^{2}}=p^{+2}+\frac{1}{2}\left(p^{2}-p_{\perp}^{2}\right) \simeq p^{+2}
$$

and

$$
\mathcal{U}_{q}^{q g}\left(p^{\prime}, k^{\prime \prime}, n\right)=-2 \frac{\left(p^{\prime} \cdot k^{\prime \prime}\right)(\gamma \cdot n)+\left(p^{\prime} \cdot n\right)\left(\gamma \cdot k^{\prime \prime}\right)}{n \cdot k^{\prime \prime}}+O\left(n^{2}\right)
$$

Here, $p$ is the four-momentum of the incoming quark which branches into $p^{\prime}$ and $k^{\prime \prime}$ of the outgoing quark and gluon, respectively. Because $n^{2} \ll 1$, the terms $O\left(n^{2}\right)$ are negligible and will be omitted in the following.

The role of the time integral $\int_{r_{0}-1 /(2 \mu)}^{r_{0}+1 /(2 \mu)} d \tau$ in Eq. (C1) over the finite time slice $\mu^{-1} \equiv \mu^{-1}(r)$ of the space-time cell around $r$ was investigated in detail in Ref. [37] to which I refer for details rather than repeating the elaborate presentation given there. For the present context it suffices to note that the evolution of the parton densities during a finite time $\Delta t=1 / \mu$ (rather than $\Delta t \rightarrow \infty)$ is modified by the time integral $\int d \tau$ weighted by the function $\mathcal{T}$ which must satisfy 


$$
\int_{0}^{\infty} d \tau \mathcal{T}\left(p^{\prime} \tau\right)=1
$$

Aside from this normalization condition, the function $\mathcal{T}$ is not determinable from first principles, but needs to be specified as physics input from plausibility arguments: to give two simple examples, $\mathcal{T}=p^{\prime} \theta\left(1-p^{\prime} \tau\right)$ or $\mathcal{T}=p^{\prime} \exp \left(-p^{\prime} \tau\right)$. As shown in Ref. [37], the essential effect of the time weight $\mathcal{T}$ is the imposition of the uncertainty principle which constrains the simultaneous use of space-time and energymomentum variables for the quantum evolution of partons. When integrated over $\tau$ as in Eq. (C1), it limits the range of virtualities $p^{\prime 2}$ such that within the finite time slice $\Delta r_{0}=\mu^{-1}$ only those fluctuations $p \leftrightarrow p^{\prime}+k^{\prime \prime}$ are resolvable that are sufficiently short living, such that

$$
\tau(p)=\gamma \tau_{0}(p)=\frac{p^{+}}{p^{\prime 2}}<\frac{1}{\mu(r)},
$$

where $\tau_{0} \simeq 1 / p^{\prime}$ is the proper lifetime of the virtual parton, to be understood in the averaged sense.

To perform the integral [Eq. (C1)], the procedure is as follows. First, due to the kinematic ordering condition $p^{2} \gg p^{\prime 2}, k^{\prime \prime 2}$ in the LLA, the gluon momentum $k^{\prime \prime}$ can be decomposed as

$$
\begin{gathered}
k^{\prime \prime}=(1-z) p-\left(\frac{1}{2}-z\right) \frac{n}{n \cdot p} p^{2}+k_{\perp}^{\prime \prime}+O\left(n^{2}\right), \\
z=\frac{p^{\prime+}}{p^{+}}, \quad k_{\perp}^{\prime \prime} \cdot p=k_{\perp}^{\prime \prime} \cdot n=0 .
\end{gathered}
$$

Then one can rewrite Eq. (C3) in the form

$\mathcal{U}_{q}^{q g}\left(p^{\prime}, k^{\prime \prime}, n\right)=2 z(\gamma \cdot p)+\left(1-z+2 z^{2}\right) \frac{(\gamma \cdot n)}{\left(n \cdot k^{\prime \prime}\right)} p^{2}$.

Next, one rewrites the integration measure in Eq. (C1) as

$$
d^{4} p^{\prime}=\frac{\pi}{2} d p^{\prime 2} d k^{\prime 2} d z \theta\left(p^{2}-\frac{p^{\prime 2}}{z}-\frac{k^{\prime \prime 2}}{1-z}\right),
$$

where the $\theta$ function accounts for the aforementioned ordering of virtualities and acts as a kinematic constraint that limits the integration range, such that

$$
k^{\prime \prime 2} \leqslant(1-z)\left(p^{2}-\frac{p^{\prime 2}}{z}\right) .
$$

Finally, to simplify the analysis, I approximate the $\tau$ integral by [37]

$$
\int_{r_{0}-1 /(2 \mu)}^{r_{0}+1 /(2 \mu)} d \tau \mathcal{T}\left(p^{\prime} \tau\right) \approx \theta\left(\frac{1}{\mu(r)}-\frac{p^{+}}{p^{\prime 2}}\right)=\theta\left(\frac{z p^{2}}{p^{+}} r_{0}-1\right),
$$

where $\mu(r)$ characterizes the size of the space-time interval of the localized quantum fluctuations [cf. Sec. III A, Eq. (50)]. Using the above formulas, the integration over $k^{\prime \prime 2}$ now gives

$$
\begin{aligned}
p^{2} \frac{\partial}{\partial p^{2}} & \hat{\Sigma}^{R(A)}(r, p) \\
= & \frac{g_{s}^{2} C_{f}}{16 \pi^{2}} \int_{0}^{1} d z \int_{0}^{z p^{2}} d p^{\prime 2}\left[2 z(\gamma \cdot p)+\left(1-z+2 z^{2}\right)\right. \\
& \left.\times\left(\frac{p^{2}(\gamma \cdot n)}{\left(n \cdot k^{\prime \prime}\right)}\right)\right] V_{q q g}^{2}\left[r ; p^{2}, p^{\prime 2},(1-z)\right. \\
& \left.\times\left(p^{2}-\frac{p^{\prime 2}}{z}\right) ; p^{+2}, z p^{+2},(1-z) p^{+2}\right] \\
& \times \Delta_{g}\left[r ;(1-z)\left(p^{2}-\frac{p^{\prime 2}}{z}\right)\right] \\
& \times \frac{\partial \Delta_{q}\left(r, p^{\prime}\right)}{\partial p^{\prime 2}} \theta\left(\frac{z p^{2}}{p^{+}} r_{0}-1\right) .
\end{aligned}
$$

Next one integrates over $p^{\prime 2}$, which yields for the last two factors in Eq. (C9) an "effective vertex function" under the remaining $z$ integral:

$$
\begin{aligned}
V_{\mathrm{eff}}^{2}= & \frac{g_{s}^{2}}{4 \pi} V_{q q g}^{2}\left[r ; p^{2}, z p^{2},(1-z) p^{2} ; p^{+2}, z p^{+2},\right. \\
& \left.(1-z) p^{+2}\right] \Delta_{q}\left(r ; z p^{2}\right) \Delta_{g}\left[r ;(1-z) p^{2}\right],
\end{aligned}
$$

which in the LLA has been shown $[27,28]$ to generate the running of the coupling $\alpha_{s}=g_{s}^{2} /(4 \pi)$. In the present case, however, it is modified by the finite-time slice effect explained after Eq. (C3), which produces the $\theta$ function $\theta\left[\left(z p^{2} / p^{+}\right) r_{0}-1\right]$ as a result of the $\int d \tau \mathcal{T}$ integral in Eq. (C3). In any case, by a nontrivial manipulation (see, e.g., [27]), Eq. (C12) can be brought into the following form, stated here without further elaboration:

$$
V_{\mathrm{eff}}^{2}=\Delta_{q}^{-1}\left(r ; p^{2}\right) \alpha_{s}\left[(1-z) p^{2}\right] \theta\left(\frac{z p^{2}}{p^{+}} r_{0}-1\right) .
$$

Employing this identification, inserting the decomposition

$$
\hat{\Sigma}^{R(A)}(r, p)=\hat{\Sigma}_{1}(\gamma \cdot p)+\hat{\Sigma}_{2} \frac{p^{2}(\gamma \cdot n)}{n \cdot p},
$$

into Eq. (C11), and solving for $\Delta_{q}$ and $\widetilde{\Delta}$ in the parametrization of the self-energy [Eq. (96)],

$$
\Delta_{q}(r, p)=\frac{1}{1+\hat{\Sigma}_{1}+\hat{\Sigma}_{2}}, \quad \widetilde{\Delta}_{q}(r, p)=\Delta_{q}\left(r ; p^{2}\right) \frac{\hat{\Sigma}_{2}}{1+\hat{\Sigma}_{1}},
$$

one finds the form of the quark renormalization function as stated in Sec. III E: 


$$
\begin{aligned}
& \Delta_{q}\left(r, p^{2}, p^{+2}\right) \\
& \quad=\exp \left\{-\int_{p^{2}}^{p^{+2}} \frac{d p^{\prime 2}}{p^{\prime 2}} \int_{0}^{1} d z A\left(r, z, p^{\prime 2}\right) \gamma_{q}^{q g}(z, \epsilon)\right\},
\end{aligned}
$$

where $^{11}$

$$
A\left(r, p^{2}, z\right) \simeq \frac{\alpha_{s}\left[(1-z) p^{2}\right]}{2 \pi} \theta\left(\frac{z p^{2}}{p^{+}}-\mu(r)\right)
$$

and

$$
\gamma_{q}^{q g}(z, \epsilon)=C_{F}\left(\frac{1+z^{2}}{1-z+\epsilon\left(p^{+2}\right)}\right)
$$

with

$$
\epsilon\left(p^{+2}\right)=\frac{p^{2} n^{2}}{4(p \cdot n)^{2}}=\frac{p^{2}}{p^{+2}} \ll 1 .
$$

By repeating the same analysis for the gluon case one obtains

$$
\begin{aligned}
\Delta_{g}\left(r, k^{2}, k^{+2}\right)= & \exp \left\{-\int_{k^{2}}^{k^{+2}} \frac{d k^{\prime 2}}{k^{\prime 2}} \int_{0}^{1} d z A\left(r, z, k^{\prime 2}\right)\right. \\
& \left.\times\left(\frac{1}{2} \gamma_{g}^{g g}(z, \epsilon)+\gamma_{g}^{q q}(z, \epsilon)\right)\right\}
\end{aligned}
$$

where

$$
\begin{gathered}
\gamma_{g}^{g g}(z, \epsilon)=2 C_{A}\left(\frac{z}{1-z+\epsilon\left(k^{+2}\right)}+\frac{1-z}{z}+z(1-z)\right), \\
\gamma_{g}^{q q}(z, \epsilon)=\frac{1}{2}\left[z^{2}+(1-z)^{2}\right] .
\end{gathered}
$$

The renormalization functions $\Delta_{f}(f=g, q)$ determine the form of the gluon and quark structure functions $\mathcal{P}_{f}^{\prime}(r, p)$ defined in Sec. III A, Eq. (61), as the spectral densities of the parton phase-space distributions $F_{f}(r, p)$, which are the space-time generalization of the usual momentum-space parton densities in QCD. With reference to the excellent review of Dokshitzer et al. [27], I merely state here the fact that the parton structure functions $\mathcal{P}_{f}^{f^{\prime}}(r, p)$ can be shown to have the following Dyson-Schwinger integral representation:

$$
\begin{aligned}
\mathcal{P}_{f}^{f^{\prime}}\left(r ; x, p^{2}\right)= & \delta_{f}^{f^{\prime}} \delta(1-x) \delta\left(p^{2}-\mu_{g q}^{2}\right) \Delta_{f}\left(r ; \mu_{g q}^{2}, p^{+2}\right) \\
& +\Delta_{f}\left(r, \mu_{g q}^{2}, p^{+2}\right) \sum_{f^{\prime \prime}} \int_{\mu_{g q}^{2}}^{2} \frac{d p^{\prime 2}}{p^{\prime 2}} \int_{0}^{1} d z \\
& \times\left\{A\left(r, p^{2}, z\right) \gamma_{f}^{f^{\prime \prime} f^{\prime}}(z, \epsilon) \mathcal{P}_{f^{\prime \prime}}^{f^{\prime}}\left(\frac{x}{z}, p^{\prime 2} ; p^{\prime+2}\right)\right. \\
& \left.\times \Delta_{f}^{-1}\left(r, \frac{p^{\prime 2}}{z}, p^{\prime+2}\right)\right\} .
\end{aligned}
$$

${ }^{11} \mathrm{~A}$ detailed derivation of the effective coupling function $A\left(r, p^{2}, z\right)$ can be found in Ref. [37].
This form can be derived by formal integration of Eq. (C11) with respect to $\ln p^{2}$, and exploiting the relation between the retarded (advanced) propagators $S^{R(A)}$ (self-energies $\Sigma^{R(A)}$ ) and the spectral densities $\mathcal{P}_{f}^{f^{\prime}}$ as given by Eq. (113). The first term on the right side of the above integral representation describes the probability that the parton density does not change. It is the product of a "free streaming" part times the Sudakov form factor $\Delta_{f} \leqslant 1$, which suppresses the free streaming probability because of the presence of parton interactions. The second term then describes the complementary probability that the parton density does actually change locally in phase space, $(r, p) \rightarrow\left(r, p^{\prime}\right)$, because of real and virtual emission and absorption processes. This second contribution is proportional to the ratio of Sudakov form factors, $\Delta_{f}\left(r, \mu_{g q}^{2}, p^{2}\right) \Delta_{f}^{-1}\left(r, p^{\prime 2} / z, p^{\prime 2}\right)$.

Now, because $F_{f}=\mathcal{N}_{f} \otimes \mathcal{P}_{f}$, Eq. (58), the variation of $F_{f}(r, p)$ with the parton momentum $p$ (more precisely, with virtuality $p^{2}$ ) therefore reflects the parton's changing gluonquark substructure as dictated by the renormalization functions, $\Delta_{g(q)}$, also called the Sudakov form factor of a gluon (quark). This connection between $\Delta_{f}$ and $F_{f}$ emerges as follows. Treating gluons and quarks on the same footing, the differentiation of $\Delta_{f}^{-1}$ with respect to $p^{2}$, the incoming partons's virtuality, yields

$$
\begin{aligned}
& p^{2} \frac{\partial}{\partial p^{2}} \Delta_{f}^{-1}\left(r ; p^{2}, p^{+2}\right) \\
& \quad=-\Delta_{f}^{-1}\left(r ; p^{2}, p^{+2}\right) \sum_{f^{\prime \prime}} \int d z A\left(r ; p^{2}, z\right) \gamma_{f}^{f^{\prime} f^{\prime \prime}}(z, \epsilon),
\end{aligned}
$$

where the sum over $f^{\prime \prime}=g, q$ automatically fixes $f^{\prime}$ due to the symmetry properties of the kernels $\gamma_{f}^{f^{\prime} f^{\prime \prime}}$ under interchange of $f^{\prime}$ and $f^{\prime \prime}$ [27]. On account of the momentum sum rule [Eq. (86)] for the parton structure functions $\mathcal{P}(r, p)=\mathcal{P}\left(r ; x, p^{2}\right)$, i.e., the momenta $p^{+}=x P^{+}(r)$ of all partons in a given cell around $r$ add up to the total cell momentum $P^{+}(r)$, one has

$$
1=\sum_{f f^{\prime}} \int d x x \mathcal{P}_{f}^{f^{\prime}}\left(r ; x, p^{2}\right),
$$

and hence

$$
\sum_{f f^{\prime}} \int d x x p^{2} \frac{\partial}{\partial p^{2}} \mathcal{P}_{f}^{f^{\prime}}\left(r ; x, p^{2}\right)=0,
$$

and, therefore, one can rewrite Eq. (C18) in the form

$$
\begin{aligned}
& {\left[p^{2} \frac{\partial}{\partial p^{2}} \Delta_{f}^{-1}\left(r ; p^{2}, p^{+2}\right)\right] \mathcal{P}_{f}^{f^{\prime}}\left(r ; x, p^{2}\right)+\Delta_{f}^{-1}\left(r ; p^{2}, p^{+2}\right)} \\
& \quad \times\left[p^{2} \frac{\partial}{\partial p^{2}} \mathcal{P}_{f}^{f^{\prime}}\left(r ; x, p^{2}\right)\right] \\
& =-\Delta_{f}^{-1}\left(r ; p^{2}, p^{+2}\right) \sum_{f^{\prime \prime}} \int d z A\left(r ; p^{2}, z\right) \\
& \quad \times \gamma_{f}^{f^{\prime} f^{\prime \prime}}(z, \epsilon) \frac{1}{z} \mathcal{P}_{f^{\prime \prime}}^{f^{\prime}}\left(r ; \frac{x}{z}, z p^{2}\right) .
\end{aligned}
$$

Employing Eq. (C23) then yields 


$$
\begin{aligned}
\Delta_{f}^{-1} & \left(r ; p^{2}, p^{+2}\right) p^{2} \frac{\partial}{\partial p^{2}} \mathcal{P}_{f}^{f^{\prime}}\left(r ; x, p^{2}\right) \\
= & \Delta_{q}^{-1}\left(r ; p^{2}, p^{+2}\right) \sum_{f^{\prime \prime}} \int d z A\left(r ; p^{2}, z\right) \gamma_{f}^{f^{\prime} f^{\prime \prime}}(z, \epsilon) \\
& \times\left\{\mathcal{P}_{f}^{f^{\prime}}\left(r ; x, p^{2}\right)-\frac{1}{z} \mathcal{P}_{f^{\prime \prime}}^{f^{\prime}}\left(r ; \frac{x}{z}, z p^{2}\right)\right\}
\end{aligned}
$$

The final evolution equation for the parton phase-space densities $F_{f}(r, p)$ is obtained by (i) multiplying with $\Delta_{f}$, (ii) convoluting the resulting equation according to Eq. (58) with the local parton density $\mathcal{N}_{f}(r, p)$, i.e., the number of dressed partons in a given cell around $r$, and (iii) accounting for the competition between real emission and reverse absorption processes [38], using the fact that the squared matrix elements $\propto \gamma_{a}^{b c}$ are invariant under the reversal $a \rightarrow b c$ and $b c \rightarrow a$. The extended result is

$$
\begin{aligned}
k^{2} \frac{\partial}{\partial k^{2}} F_{g}\left(r ; x, k^{2}\right)= & \int_{0}^{1} d z A\left(r ; k^{2}, z\right)\left\{\left[\frac{1}{z} F_{g}\left(r ; \frac{x}{z}, z k^{2}\right)\right.\right. \\
& \left.-\frac{1}{2} F_{g}\left(r ; x, k^{2}\right)\right] \Gamma_{g}^{g g}(z, \epsilon) \\
& +2 N_{f} F_{q}\left(r ; x, k^{2}\right) \Gamma_{q}^{g q}(z, \epsilon) \\
& \left.-N_{f} F_{g}\left(r ; x, k^{2}\right) \Gamma_{g}^{q q}(z, \epsilon)\right\}, \\
p^{2} \frac{\partial}{\partial p^{2}} F_{q}\left(r ; x, p^{2}\right)= & \int_{0}^{1} d z A\left(r ; p^{2}, z\right)\left\{\left[\frac{1}{z} F_{q}\left(r ; \frac{x}{z}, z p^{2}\right)\right.\right. \\
& \left.-F_{q}\left(r ; x, p^{2}\right)\right] \Gamma_{q}^{q g}(z, \epsilon) \\
& \left.+F_{g}\left(r ; x, p^{2}\right) \Gamma_{g}^{q q}(z, \epsilon)\right\},
\end{aligned}
$$

where

$$
\Gamma_{f}^{f^{\prime} f^{\prime \prime}}=\gamma_{f}^{f^{\prime} f^{\prime \prime}}\left(1-\frac{F_{f^{\prime}}}{F_{f^{\prime}} \pm 1}\right)
$$

represents the net emission probability, being a manifestation of the principle of detailed balance between the rate of emission of parton $f^{\prime}$ from a parton $f$, and the rate of absorption of a quantum $f^{\prime}$ in the phase-space proximity of parton $f$. The net rate $\Gamma_{f}^{f^{\prime} f^{\prime \prime}}$ results in a suppression when $F_{g}$ or $F_{q}$ becomes large, and thus reflects correctly the Bose-Einstein and Fermi-Dirac statistics (+ for gluons, - for quarks). On the other hand, when $F_{g}$ and $F_{q}$ are small compared to 1 , the usual branching kernels $\gamma_{f}^{f^{\prime} f^{\prime \prime}}$ are recovered.

\section{APPENDIX D: DERIVATION OF DRIFT TERM AND COLLISION KERNEL OF THE TRANSPORT EQUATIONS}

This appendix explains the derivation of the transport equations (115) and (127) that govern the kinetic, dispersive dynamics of dressed gluons and quarks. First of all, one observes that from the matrix representation of the CTP Dyson-
Schwinger equations (39) and (40) in terms of the Green functions $G^{F}, G^{\bar{F}}, G^{>}$, and $G^{<}$and self-energies $\mathcal{E}^{F}, \mathcal{E}^{\bar{F}}, \mathcal{E}^{>}$, and $\mathcal{E}^{<}$, where $G \equiv D_{\mu \nu}, S$ and $\mathcal{E}=\Pi_{\mu \nu}, \Sigma$, follow immediately the corresponding equations for the retarded, advanced, and correlation functions $G^{R}, G^{A}$, and $G^{C}$ and $\mathcal{E}^{R}, \mathcal{E}^{A}$, and $\mathcal{E}^{C}$, as given by Eq. (93):

$$
\begin{gathered}
\left(D_{R(A)}^{-1}\right)^{\mu \nu}(r, k)=\left(D_{(0) R(A)}^{-1}\right)^{\mu \nu}-\left(\Pi_{R(A)}\right)^{\mu \nu}, \\
S_{R(A)}^{-1}(r, p)=S_{(0) R(A)}^{-1}-\Sigma_{R(A)},
\end{gathered}
$$

and

$$
\begin{gathered}
D_{C}^{\mu \nu}(r, k)=-D_{R}^{\mu \mu^{\prime}}\left[\left(D_{(0) C}^{-1}\right)^{\mu^{\prime} \nu^{\prime}}-\left(\Pi_{C}\right)^{\mu^{\prime} \nu^{\prime}}\right] D_{A}^{\nu^{\prime} \nu} \\
S_{C}(r, p)=-S_{R}\left[S_{(0) C}^{-1}-\Sigma_{C}\right] S_{A}
\end{gathered}
$$

for the quarks. It is convenient to introduce scalar and dimensionless self-energy functions $\hat{\Pi}$ and $\hat{\Sigma}$ through

$$
\begin{gathered}
\Pi_{a b}^{\mu \nu}(r, k)=\delta_{a b}\left(k^{\mu} k^{\nu}-g^{\mu \nu} k^{2}\right) \hat{\Pi}(r, k), \\
\Sigma_{i j}(r, p)=\delta_{i j} p^{2} \hat{\Sigma}(r, p),
\end{gathered}
$$

so that the propagators can be written as

$$
\begin{gathered}
D_{a b}^{\mu \nu R(A)}(r, k)=\delta_{a b}\left(-d_{\mu \nu}(k)\right) \frac{1}{\pi_{0} \pm i \pi_{1}}, \\
S_{i j}^{R(A)}(r, p)=\delta_{i j}(\gamma \cdot p) \frac{1}{\sigma_{0} \pm \sigma_{1}},
\end{gathered}
$$

and the correlation functions as

$$
\begin{gathered}
D_{a b}^{\mu \nu C}(r, k)=\delta_{a b}\left(-d_{\mu \nu}(k)\right) \frac{-2 i \pi_{2}}{\pi_{0}^{2}+\pi_{1}^{2}}\left[1+F_{g}\right], \\
S_{i j}^{C}(r, p)=\delta_{i j}(\gamma \cdot p) \frac{-2 i \sigma_{2}}{\sigma_{0} \pm \sigma_{1}}\left[1-F_{q}\right],
\end{gathered}
$$

where $d_{\mu \nu}(k)$ is defined in Eq. (8), and $F_{g}$ and $F_{q}$ are the phase-space densities of dressed gluons and quarks as defined by Eq. (58). This separation of real and imaginary contributions uniquely determines the Green functions in terms of the three functions $\pi_{i}\left(\sigma_{i}\right)$ as can be shown rigorously [7]. The real parts correspond to the dispersive and wave function renormalization piece, whereas the imaginary parts give rise to dissipation and decay. Formally,

$$
\begin{gathered}
\pi_{0}=k^{2}(1-\operatorname{Re} \hat{\Pi}), \quad \operatorname{Re} \hat{\Pi}=\frac{1}{2}\left(\hat{\Pi}^{R}+\hat{\Pi}^{A}\right), \\
\pi_{1}=-k^{2} \operatorname{Im} \hat{\Pi}, \quad \operatorname{Im} \hat{\Pi}=\frac{i}{2}\left(\hat{\Pi}^{R}-\hat{\Pi}^{A}\right), \\
\sigma_{0}=p^{2}(1-\operatorname{Re} \hat{\Sigma}), \quad \operatorname{Re} \hat{\Sigma}=\frac{1}{2}\left(\hat{\Sigma}^{R}+\hat{\Sigma}^{A}\right), \\
\sigma_{1}=-p^{2} \operatorname{Im} \hat{\Sigma}, \quad \operatorname{Im} \hat{\Sigma}=\frac{i}{2}\left(\hat{\Sigma}^{R}-\hat{\Sigma}^{A}\right),
\end{gathered}
$$


and

$$
\pi_{2}=\frac{i}{2}\left(\hat{\Pi}^{<}+\hat{\Pi}^{>}\right), \quad \sigma_{2}=\frac{i}{2}\left(\hat{\Sigma}^{<}+\hat{\Sigma}^{>}\right)
$$

Next, recalling that the correlations among differently dressed partons determine their mutual interactions at kinetic space-time scales, one focuses on the correlation functions $D_{\mu \nu}^{C}$ and $S^{C}$. Noting that $D_{\mu \nu}^{A}=D_{\mu \nu}^{R \dagger}, S^{A}=S^{R^{\dagger}}$, and employing the representations (92),

$$
\begin{gathered}
D_{\mu \nu}^{C}(r, k)=-2 \pi i\left[-d_{\mu \nu}(k)\right]\left[1+2 F_{g}(r, k)\right] \\
\times \delta\left(k^{2}-\mathcal{M}_{g}^{2}(r, k)\right), \\
S^{C}(r, p)=-2 \pi i(\gamma \cdot p)\left[1-2 F_{q}(r, p)\right] \delta\left(p^{2}-\mathcal{M}_{q}^{2}(r, p)\right),
\end{gathered}
$$

where color indices are suppressed, Eqs. (D4)-(D8) may be combined to write

$$
\begin{aligned}
-d^{\mu \nu}(k) D_{\mu \nu}^{C}(r, k) & =\left(\pi_{0}+i \pi_{1}\right)^{-1} H_{g}-H_{g}\left(\pi_{0}-i \pi_{1}\right)^{-1} \\
& =-2 i \frac{\pi_{2}}{\pi_{0}^{2}+\pi_{1}^{2}}, \\
\gamma \cdot p S^{C}(r, p) & =\left(\sigma_{0}+i \sigma_{1}\right)^{-1} H_{q}-H_{q}\left(\sigma_{0}-i \sigma_{1}\right)^{-1} \\
& =-2 i \frac{\sigma_{2}}{\sigma_{0}^{2}+\sigma_{1}^{2}},
\end{aligned}
$$

where [cf. Eq. (89)]

$$
H_{g}(r, k)=1+2 F_{g}(r, k), \quad H_{q}(r, p)=1-2 F_{q}(r, p) \text {. }
$$

Hence, on account of Eqs. (D1) and (D2) one finds

$$
\begin{gathered}
-d^{\mu \nu}(k)\left(D_{(0)}^{C-1}-\Pi^{C}\right)_{\mu \nu}= \\
+\left(H_{g} \pi_{0}-\pi_{0} H_{g}\right) \\
+i\left(H_{g} \pi_{1}+\pi_{1} H_{g}\right), \\
\gamma \cdot p\left(S_{(0)}^{C-1}-\Sigma^{C}\right)=-\left(H_{q} \sigma_{0}-\sigma_{0} H_{q}\right)+i\left(H_{q} \sigma_{1}+\sigma_{1} H_{q}\right) .
\end{gathered}
$$

Then, by inserting the expressions (D7) and (D8) for $\pi_{i}$ and $\sigma_{i}$, one obtains

$$
\begin{aligned}
F_{g} \pi_{0}-\pi_{0} F_{g}= & \frac{1}{2}\left[\left(1+F_{g}\right) \hat{\Pi}^{<}+\hat{\Pi}^{<}\left(1+F_{g}\right)\right. \\
& \left.-F_{g} \hat{\Pi}^{>}-\hat{\Pi}^{>} F_{g}\right] \\
\equiv & \mathcal{C}_{g}, \\
F_{q} \sigma_{0}-\sigma_{0} F_{q}= & -\frac{1}{2}\left[\left(1-F_{q}\right) \hat{\Sigma}^{<}+\hat{\Sigma}^{<}\left(1-F_{q}\right)\right. \\
& \left.+F_{q} \hat{\Sigma}^{>}+\hat{\Sigma}^{>} F_{q}\right] \\
\equiv & \mathcal{C}_{q},
\end{aligned}
$$

with the self-energies $\hat{\Pi}^{\gtrless}$ and $\hat{\Sigma}^{\gtrless}$ given by Eqs. (121) and (122) together with Eq. (D3). Finally, as argued in Sec. III E, the presumed clear separation of quantum and kinetic spacetime scales allows one to treat the kinetic dynamics quasiclassically, by expanding both sides of Eq. (D13) in terms of $\hbar$ and keeping only the lowest-order contributions. The lowest-order nonzero terms on the right hand sides of Eq. (D13) correspond then to the Born collision terms which are of order $\hbar$,

$$
\begin{gathered}
\mathcal{C}_{g}(r, k)=\left(1+F_{g}\right) \hat{\Pi}^{<}-F_{g} \hat{\Pi}^{>}+O\left(\hbar^{3}\right), \\
\mathcal{C}_{q}(r, p)=-\left(1-F_{q}\right) \hat{\Sigma}^{<}+F_{q} \hat{\Sigma}^{>}+O\left(\hbar^{3}\right),
\end{gathered}
$$

whereas the lowest-order nonzero terms on the left hand sides of Eq. (D13) result in the classical Poisson brackets which are also of order $\hbar$,

$$
\begin{aligned}
\left(F_{g} \pi_{0}-\pi_{0} F_{g}\right)(r, k)= & -i\left[\left(\partial_{r} F_{g}\right) \cdot\left(\partial_{k} \pi_{0}\right)-\left(\partial_{k} F_{g}\right) \cdot\left(\partial_{r} \pi_{0}\right)\right] \\
= & -i\left(\frac{\partial \pi_{0}(r, k)}{\partial k^{2}}\right)_{k^{2}=\mu_{g q}^{2}}\left(k \cdot \partial_{r} F_{g}(r, k)\right. \\
& \left.-\frac{1}{2} \partial_{r} k_{0}^{2} \cdot \partial_{k} F_{g}(r, k)\right)+O\left(\hbar^{3}\right), \\
\left(F_{q} \sigma_{0}-\sigma_{0} F_{q}\right)(r, p)= & -i\left[\left(\partial_{r} F_{q}\right) \cdot\left(\partial_{p} \sigma_{0}\right)-\left(\partial_{p} F_{q}\right) \cdot\left(\partial_{r} \sigma_{0}\right)\right] \\
= & -i\left(\frac{\partial \sigma_{0}(r, p)}{\partial p^{2}}\right)_{p^{2}=\mu_{g q}^{2}}\left(p \cdot \partial_{r} F_{q}(r, p)\right. \\
& \left.-\frac{1}{2} \partial_{r} p_{0}^{2} \cdot \partial_{p} F_{q}(r, p)\right)+O\left(\hbar^{3}\right),
\end{aligned}
$$

where as before $\partial_{r}=\partial / \partial r^{\mu}, \partial_{k}=\partial / \partial k^{\mu}$, etc., and the center dot denotes a scalar product of four-vectors. The latter equalities in these two equations are obtained by using the fact that the solutions of the dressed partons' energy spectra Eq. (124) are strongly peaked around momentum transfers $q_{\perp}^{2} \simeq \mu_{g q}^{2}$, i.e., $\quad k_{0}(r, \vec{k}) \simeq \sqrt{\vec{k}^{2}+\mu_{g q}^{2}} \quad$ and $\quad p_{0}(r, \vec{p})$ $\simeq \sqrt{\vec{p}^{2}+\mu_{g q}^{2}}$, because of the well-known QCD specific logarithmic behavior of the spectral densities $\propto \alpha_{s} \ln \left(q_{\perp}^{2} / \mu_{g q}^{2}\right)$, and the power law form of the scattering cross sections $\propto \alpha_{s}^{2} q_{\perp}^{-n}(n \simeq 4)$.

Finally, using $\partial_{r} k_{0}=\partial_{r} p_{0} \approx 0$, and equating Eqs. (D13) and (D15), one obtains the transport equations of Boltzmann type, stated in Sec. III E, Eqs. (120),

$$
k \cdot \partial_{r} F_{g}(r, k)=\mathcal{I}_{g}(r, k), \quad p \cdot \partial_{r} F_{q}(r, p)=\mathcal{I}_{q}(r, p),
$$

where the Lorentz-invariant collision terms $\mathcal{I}$ on the right hand side are defined by

$$
\begin{aligned}
& \mathcal{C}_{g}(r, k)=-i\left(\frac{\partial \pi_{0}(r, k)}{\partial k^{2}}\right)_{k^{2}=\mu_{g q}^{2}} \mathcal{I}_{g}(r, k), \\
& \mathcal{C}_{q}(r, p)=-i\left(\frac{\partial \sigma_{0}(r, p)}{\partial p^{2}}\right)_{p^{2}=\mu_{g q}^{2}} \mathcal{I}_{q}(r, p) .
\end{aligned}
$$

In Eqs. (D15) and (D17), the derivatives with respect to the virtuality $k^{2}$ are to be taken at $\mu_{g q}^{2}$, which according to Eqs. (56) and (98) defines the scale at which a dressed parton 
appears as a quasiparticle, with the renormalization and dissipation effects taken into account in the spectral densities $\mathcal{P}_{g}$ and $\mathcal{P}_{q}$, Eq. (114). Their form is determined by the renormalization equations (115), and therefore the parton distributions $F=\mathcal{N} \otimes \mathcal{P}$ contain implicitly the short-distance quantum effects. The derivatives of $\pi_{0}$ and $\sigma_{0}$ are related to the renormalization functions $\Delta_{g}$ and $\Delta_{q}$, respectively, via the correspondence of the representations (D4) and (96), and one finds

$$
\begin{aligned}
& -i\left(\frac{\partial \pi_{0}(r, k)}{\partial k^{2}}\right)_{k^{2}=\mu_{g q}^{2}}=\left.\Delta_{g}^{-1}\left(r, k^{2}, k^{+2}\right)\right|_{k^{2}=\mu_{g q}^{2}}=1, \\
& -i\left(\frac{\partial \sigma_{0}(r, p)}{\partial p^{2}}\right)_{p^{2}=\mu_{g q}^{2}}=\left.\Delta_{q}^{-1}\left(r, p^{2}, p^{+2}\right)\right|_{p^{2}=\mu_{g q}^{2}}=1,
\end{aligned}
$$

where the latter equality results from the normalization condition (98).

The explicit form of the collision integral $\mathcal{I}$ is obtained by substituting the correlation functions (D9) into the two-loop expressions (121) and (122) for the self-energies $\hat{\Pi}$ and $\hat{\Sigma}$, and then inserting those into Eqs. (D13). Applying the standard cutting rules [42] to the resulting self-energies, as symbolically represented in Fig. 11, yields the different binary collision processes $a b \leftrightarrow c d$ by which a parton of type $a$ may be gained or lost in a phase-space element, namely, $g g \leftrightarrow g g, g g \leftrightarrow q \bar{q}, g q \leftrightarrow g q, q q \leftrightarrow q q, q \bar{q} \leftrightarrow q \bar{q}$. The corresponding collision integrals $\mathcal{I}_{a}$ may be compactly represented in the generic form of Eq. (130):

$$
\begin{aligned}
\mathcal{I}_{a}\left(r, p_{1}\right) \equiv & \sum_{b c d}\left[-\mathcal{I}_{c d \rightarrow a b}^{\text {(loss) }}\left(p_{1}, r\right)+\mathcal{I}_{a b \rightarrow c d}^{\text {(gain })}\left(p_{1}, r\right)\right] \\
= & -\sum_{b c d} C_{a b} C_{c d} \int \frac{d^{3} p_{2}}{(2 \pi)^{3} 2 E_{2}} \int \frac{d^{3} p_{3}}{(2 \pi)^{3} 2 E_{3}} \\
& \times \int \frac{d^{3} p_{4}}{(2 \pi)^{3} 2 E_{4}}(2 \pi)^{4} \delta^{4}\left(p_{1}+p_{2}-p_{3}-p_{4}\right) \\
& \times\left\{F_{a}(1) F_{b}(2)|\mathcal{M}(a b \rightarrow c d)|^{2} \theta\left(q_{\perp}^{2}-\mu_{g q}^{2}\right)\right. \\
& \times\left[1 \pm F_{c}(3)\right]\left[1 \pm F_{d}(4)\right]-\left[1 \pm F_{a}(1)\right] \\
& \times\left[1 \pm F_{b}(2)\right]|\mathcal{M}(c d \rightarrow a b)|^{2} \\
& \left.\times \theta\left(q_{\perp}^{2}-\mu_{g q}^{2}\right) F_{c}(3) F_{d}(4)\right\},
\end{aligned}
$$

where the $F_{\alpha}(i) \equiv F_{\alpha}\left(p_{i}, r\right)$ denote the distribution functions of parton species $\alpha=a, b, c, d$ and corresponding fourmomenta $p_{i}=p_{1}, p_{2}, p_{3}, p_{4}$ at space-time point $r=\left(r^{0}, \vec{r}\right)$. As a consequence of the representations (D9), the squared matrix elements $|\mathcal{M}|^{2}$ for the processes $a b \leftrightarrow c d$ (which contain the $2 \rightarrow 2$ kinematics, color, and spin structure, as given below) are weighted by a distribution function $F_{\alpha}$ for each of the particles coming into the interaction vertex and a factor $\left[1 \pm F_{\alpha}\right]$ for each of the outgoing ones, with the + sign referring to gluons and the - sign to quarks and antiquarks. The factors $S_{a b}=\left(1+\delta_{a b}\right)^{-1}$ and $S_{c d} \equiv\left(1+\delta_{c d}\right)^{-1}$ account for the cases where the two incoming and/or outgoing partons are identical. Using the identities ${ }^{12}$

$$
\begin{gathered}
d_{\mu \nu}(k)=\sum_{s=1,2} \varepsilon_{\mu}(k, s) \cdot \varepsilon_{\nu}^{*}(k, s), \\
\gamma \cdot p=2 p_{0} \sum_{s=1,2} \bar{u}(p, s) u(p, s)=2 p_{0} \sum_{s=1,2} \bar{v}(p, s) v(p, s),
\end{gathered}
$$

finally, the squared matrix elements are obtained by evaluating the amplitudes illustrated in Fig. 11, squaring those, averaging over initial colors and spins, summing over final colors and spins, and summing over quark flavors. The resulting expressions are standard and given by

$$
\begin{aligned}
\mid \mathcal{M}\left(g_{a} g_{b} \rightarrow\right. & \left.g_{c} g_{d}\right)\left.\right|^{2}=\frac{g_{s}^{4}}{(8 \times 2)^{2}} \sum_{\text {color, spin }} \mid \frac{g_{\tau \tau^{\prime}} f_{a e d} f_{e b c}}{\left(p_{1}-p_{4}\right)^{2}} \lambda^{\rho \tau \sigma}\left(-p_{1}, p_{1}-p_{4}, p_{4}\right) \lambda^{\tau^{\prime} \mu \nu}\left(p_{2}-p_{3},-p_{2}, p_{3}\right) \\
& +\frac{g_{\tau \tau^{\prime}} f_{a e c} f_{e b d}}{\left(p_{1}-p_{3}\right)^{2}} \lambda^{\rho \tau \nu}\left(-p_{1}, p_{1}-p_{3}, p_{3}\right) \lambda^{\tau^{\prime} \mu \sigma}\left(p_{2}-p_{4},-p_{2}, p_{4}\right) \\
& +\frac{g_{\tau \tau^{\prime}} f_{d b e} f_{e c d}}{\left(p_{1}+p_{2}\right)^{2}} \lambda^{\rho \mu \tau}\left(-p_{1},-p_{2}, p_{1}+p_{2}\right) \lambda^{\tau^{\prime} \nu \sigma}\left(-p_{3}-p_{4}, p_{3}, p_{4}\right)+\left.v_{a b c d}^{\rho \mu \nu \sigma}\left(p_{1}, p_{2},-p_{3},-p_{4}\right)\right|^{2},
\end{aligned}
$$

\footnotetext{
${ }^{12}$ Note that in contrast to the standard normalization for fermions $\propto \sqrt{m / p^{0}}$, here the normalization is chosen commonly for both gluons and quarks $\propto 1 /\left(2 p^{0}\right)$.
} 


$$
\begin{aligned}
& \left|\mathcal{M}\left(g_{a} g_{b} \rightarrow \overline{q_{i}} q_{j}\right)\right|^{2}=\frac{g_{s}^{4}}{(8 \times 2)} \sum_{f}^{N_{f}} \sum_{\text {color, spin }} \mid \bar{u}_{j}\left(p_{4}\right)\left(T_{i k}^{a} T_{k j}^{b} \gamma \cdot \varepsilon\left(p_{2}\right) \frac{\gamma \cdot\left(p_{1}-p_{3}\right)}{\left(p_{1}-p_{3}\right)^{2}} \gamma \cdot \varepsilon\left(p_{1}\right)\right) v_{i}\left(p_{3}\right) \\
& +\bar{u}_{j}\left(p_{4}\right)\left(T_{i k}^{b} T_{k j}^{a} \gamma \cdot \varepsilon\left(p_{1}\right) \frac{\gamma \cdot\left(p_{2}-p_{3}\right)}{\left(p_{2}-p_{3}\right)^{2}} \gamma \cdot \varepsilon\left(p_{2}\right)\right) v_{i}\left(p_{3}\right) \\
& +\left.\bar{u}_{j}\left(p_{4}\right)\left(i f^{a b c} T_{i j}^{c} \frac{\varepsilon^{\mu}\left(p_{1}\right) \varepsilon^{\nu}\left(p_{2}\right) \gamma^{\rho}}{\left(p_{1}+p_{2}\right)^{2}} \lambda_{\mu \nu \rho}\left(-p_{1},-p_{2}, p_{1}+p_{2}\right)\right) v_{i}\left(p_{3}\right)\right|^{2}, \\
& \left|\mathcal{M}\left(g_{a} q_{i} \rightarrow g_{b} q_{j}\right)\right|^{2}=\frac{g_{s}^{4}}{(8 \times 2)(3 \times 2)} \sum_{f}^{N_{f}} \sum_{\text {color,spin }} \mid f^{c a b} T_{i j}^{c} \frac{\varepsilon^{\mu}\left(p_{1}\right) \varepsilon^{\nu}\left(p_{3}\right)}{\left(p_{1}-p_{3}\right)^{2}} \lambda_{\rho \mu \nu}\left(p_{1}-p_{3},-p_{1}, p_{3}\right) \bar{u}_{j}\left(p_{4}\right) \gamma^{\rho} u_{i}\left(p_{2}\right) \\
& -i T_{i k}^{b} T_{k j}^{a} \bar{u}_{j}\left(p_{4}\right) \gamma \cdot \varepsilon\left(p_{1}\right) \frac{\gamma \cdot\left(p_{2}-p_{3}\right)}{\left(p_{2}-p_{3}\right)^{2}} \gamma \cdot \varepsilon\left(p_{3}\right) u_{i}\left(p_{2}\right) \\
& -\left.i T_{i l}^{a} T_{l j}^{b} \bar{u}_{j}\left(p_{4}\right) \gamma \cdot \varepsilon^{\mu}\left(p_{3}\right) \frac{\gamma \cdot\left(p_{2}+p_{1}\right)}{\left(p_{2}+p_{1}\right)^{2}} \gamma \cdot \varepsilon\left(p_{1}\right) u_{i}\left(p_{2}\right)\right|^{2} \\
& \left|\mathcal{M}\left(q_{i} q_{k} \rightarrow q_{j} q_{l}\right)\right|^{2}=\frac{g_{s}^{4}}{(3 \times 2)^{2}} \sum_{f_{1}, f_{2}}^{N_{f}} \sum_{\text {color, spin }} \mid T_{i j}^{a} T_{k l}^{a} \bar{u}_{j}\left(p_{4}\right) \gamma_{\mu} u_{i}\left(p_{1}\right) \frac{1}{\left(p_{1}-p_{4}\right)^{2}} \bar{u}_{l}\left(p_{3}\right) \gamma^{\mu} u_{k}\left(p_{2}\right) \\
& -\left.\delta_{f_{1} f_{2}} T_{i l}^{b} T_{k j}^{b} \bar{u}_{j}\left(p_{4}\right) \gamma_{\nu} u_{k}\left(p_{2}\right) \frac{1}{\left(p_{1}-p_{3}\right)^{2}} \bar{u}_{l}\left(p_{3}\right) \gamma_{\nu} u_{i}\left(p_{1}\right)\right|^{2} \\
& \left|\mathcal{M}\left(q_{i} \bar{q}_{k} \rightarrow q_{j} \overline{q_{l}}\right)\right|^{2}=\frac{g_{s}^{4}}{3 \times 2 \times 2} \sum_{f_{1}, f_{2}}^{N_{f}} \sum_{\text {color, spin }} \mid \delta_{f_{1} f_{4}} \delta_{f_{2} f_{3}} T_{i j}^{a} T_{l k}^{a} \bar{u}_{j}\left(p_{4}\right) \gamma_{\mu} u_{i}\left(p_{1}\right) \frac{1}{\left(p_{1}-p_{4}\right)^{2}} \bar{v}_{k}\left(p_{2}\right) \gamma^{\mu} v_{l}\left(p_{3}\right) \\
& -\left.\delta_{f_{1} f_{2}} \delta_{f_{4} f_{3}} T_{i k}^{b} T_{l i}^{b} \bar{u}_{j}\left(p_{4}\right) \gamma_{\nu} v_{l}\left(p_{3}\right) \frac{1}{\left(p_{1}-p_{3}\right)^{2}} \bar{v}_{k}\left(p_{2}\right) \gamma_{\nu} u_{i}\left(p_{1}\right)\right|^{2}, \\
& \left|\mathcal{M}\left(\bar{q}_{i} q_{j} \rightarrow g_{a} g_{b}\right)\right|^{2}=\frac{64}{9} \mid \mathcal{M}\left(\left.g_{a} g_{b} \rightarrow \overline{q_{i}} q_{j}\right|^{2} .\right.
\end{aligned}
$$

Here

$$
\begin{gathered}
\lambda^{\mu \rho \nu}\left(p_{1}, p_{2}, p_{3}\right) \equiv\left(p_{1}-p_{2}\right)^{\nu} g^{\mu \rho}+\left(p_{2}-p_{3}\right)^{\mu} g^{\rho \nu}+\left(p_{3}-p_{1}\right)^{\rho} g^{\mu \nu} \\
v_{a b c d}^{\mu \sigma \tau \nu}\left(p_{1}, p_{2}, p_{3}, p_{4}\right) \equiv f_{a b e} f_{c d e}\left(g^{\rho \nu} g^{\mu \sigma}-g^{\rho \sigma} g^{\mu \nu}\right)+f_{a c e} f_{b d e}\left(g^{\rho \mu} g^{\nu \sigma}-g^{\rho \sigma} g^{\mu \nu}\right)+f_{a d e} f_{c d e}\left(g^{\rho \nu} g^{\mu \sigma}-g^{\rho \mu} g^{\sigma \nu}\right)
\end{gathered}
$$

are the usual three-gluon vertex function and the four-gluon vertex, respectively. The shorthand notation suppressing spinor and polarization indices, $u\left(p_{1}\right) \equiv u\left(p_{1}, s_{1}\right)_{\alpha}, \varepsilon\left(p_{2}\right) \equiv \varepsilon\left(p_{2}, s_{2}\right)$, etc., is employed, and in Eqs. (D24) and (D25) $\delta_{f f}$, is equal to 1 if the two quarks are of the same flavor and is zero otherwise.

[1] See, e.g., B. Webber, in Proceedings of the Summer School on Hadronic Aspects of Collider Physics, Zuoz, Switzerland, 1994, edited by M. Locker (PSI, Villigen, 1994).

[2] J. Ellis and K. Geiger, Phys. Rev. D 52, 1500 (1995); see also Report No. CERN-TH. 95-283 (hep-ph/9511321) (unpublished).

[3] J. Schwinger, J. Math. Phys. 2, 407 (1961).

[4] L. V. Keldysh, Sov. Phys. JETP 20, 1018 (1965).

[5] L. P. Kadanoff and G. Baym, Quantum Statistical Mechanics (Benjamin, New York, 1962).

[6] K. T. Mahanthappa, Phys. Rev. 126, 329 (1962); P. M. Bakshi and K. T. Mahanthappa, J. Math. Phys. 4, 1 (1961); 4, 12 (1961).

[7] K. C. Chou, Z.-B. Su, B.-L. Hao, and L. Yu, Phys. Rep. 118, 1 (1985).

[8] E. Calzetta and B. L. Hu, Phys. Rev. D 37, 2887 (1988).

[9] J. Rammer and H. Smith, Rev. Mod. Phys. 58, 323 (1986).

[10] C. de Dominicis and P. Martin, J. Math. Phys. 5, 14 (1964); 5, 31 (1964).

[11] J. M. Cornwall, R. Jackiw, and E. Tomboulis, Phys. Rev. D 10, 2428 (1974).

[12] R. D. Jordan, Phys. Rev. D 33, 44 (1986). 
[13] E. P. Wigner, Phys. Rev. 40, 749 (1932).

[14] M. Hillery, R. F. O'Connell, M. O. Scully, and E. P. Wigner, Phys. Rep. 106, 121 (1984).

[15] S. de Groot, W. A. van Leuwen, and C. G. van Weert, Relativistic Kinetic Theory (North-Holland, Amsterdam, 1980).

[16] H.-T. Elze, M. Gyulassy, and D. Vasak, Nucl. Phys. B276, 706 (1986); Phys. Lett. B 177, 402 (1986).

[17] H.-T. Elze and U. Heinz, Phys. Rep. 183, 81 (1989).

[18] H.-T. Elze, Z. Phys. C 47, 647 (1990).

[19] K. Konishi, A. Ukawa, and G. Veneziano, Nucl. Phys. B157, 45 (1979).

[20] A. Basetto, M. Ciafaloni, and G. Marchesini, Nucl. Phys. B163, 477 (1980).

[21] S. Li and L. McLerran, Nucl. Phys. B214, 417 (1983).

[22] W.-M. Zhang and L. Wilets, Phys. Rev. C 45, 1900 (1992).

[23] P. Danielewicz, Ann. Phys. (N.Y.) 152, 238 (1984); 152, 305 (1984); 197, 154 (1990).

[24] S. Mrówczyński and U. Heinz, Ann. Phys. (N.Y.) 229, 1 (1993); 229, 1 (1994).

[25] L. McLerran and R. Venugopalan, Phys. Rev. D 49, 2233 (1994); 49, 3352 (1994); A. Ayala, J. Jalilian-Marian, L. McLerran, and R. Venugopalan, ibid. 52, 2935 (1995); A. Kovner, L. McLerran, and H. Weigert, ibid. 52, 6231 (1995).

[26] A. Makhlin, Phys. Rev. C 51, 3454 (1995); 52, 995 (1995).

[27] Yu. L. Dokshitzer, D. I. Dyakonov, and S. I. Troyan, Phys. Rep. 58, 269 (1980).

[28] D. Amati, A. Bassetto, M. Ciafaloni, G. Marchesini, and G. Veneziano, Nucl. Phys. B173, 429 (1980).

[29] A. H. Mueller, Phys. Rep. 73, 237 (1981).

[30] Perturbative Quantum Chromodynamics, edited by A. H. Mueller, Advanced Series on Directions in High Energy Phys- ics Vol. 5 (World Scientific, Singapore, 1989).

[31] Yu. L. Dokshitzer, V. A. Khoze, A. H. Mueller, and S. I. Troyan, Basics of Perturbative QCD, Advanced Series on Directions in High Energy Physics Vol. 5 (Editions Frontieres, Gif-sur-Yvette, France, 1991).

[32] A. Bassetto, G. Nardelli, and R. Soldati, Yang-Mills Theories in Algebraic Non-Covariant Gauges (World Scientific, Singapore, 1991).

[33] Yu. L. Dokshitzer and S. I. Troyan, Rev. Mod. Phys. 60, 373 (1988).

[34] V. L. Ginzburg and L. D. Landau, Zh. Eksp Teor. Fiz. 20, 1064 (1950).

[35] F. J. Dyson, Phys. Rev. 75, 1736 (1949); J. Schwinger, ibid. 75, 651 (1949).

[36] L. V. Gribov, E. M. Levin, and M. G. Ryskin, Phys. Rep. 100, 1 (1983).

[37] K. Geiger and B. Müller, Phys. Rev. D 50, 337 (1994); K. Geiger, ibid. 50, 3243 (1994).

[38] K. Geiger, Phys. Rev. D 46, 4965 (1992).

[39] See, e.g., J. C. Collins, D. E. Soper, and G. Sterman, in Ref. [30]; CTEQ Collaboration, R. Broch et al., Rev. Mod. Phys. 67, 157 (1995).

[40] P. Henning, Phys. Rep. 253, 235 (1995).

[41] J. Rau and B. Müller, Report No. Duke-THT-95-92, 1995 (unpublished).

[42] R. E. Cutkosky, Rev. Mod. Phys. 33, 446 (1961); P. D. B. Collins, An Introduction to Regge Theory and High-Energy Physics (Cambridge University Press, Cambridge, England 1977).

[43] K. Geiger, Phys. Rep. 258, 237 (1995). 